\title{
O algoritmo de simulação estocástica para o estudo do comportamento da epidemia de dengue em sua fase inicial
}

\author{
Anderson Tamotsu Nakashima
}

\author{
DissERTAÇÃo APRESENTADA \\ $\mathrm{AO}$ \\ Instituto DE MATEMÁticA E Estatística \\ DA \\ Universidade DE SÃo PAUlo \\ PARA \\ OBTENÇÃO DO TÍTULO \\ DE \\ Mestre EM CiênCIAS \\ Programa: Matemática Aplicada \\ Orientador: Prof. Dr. Nelson Mugayar Kuhl
}

Durante o desenvolvimento deste trabalho o autor recebeu auxílio financeiro da CAPES

São Paulo, 24 de agosto de 2018 


\section{O algoritmo de simulação estocástica para o estudo do comportamento da epidemia de dengue em sua fase inicial}

Esta versão da dissertação contém as correções e alterações sugeridas pela Comissão Julgadora durante a defesa da versão original do trabalho, realizada em 24/08/2018. Uma cópia da versão original está disponível no

Instituto de Matemática e Estatística da Universidade de São Paulo.

Comissão Julgadora:

- Prof. Dr. Nelson Mugayar Kuhl (orientador) - IME-USP

- Prof. Dr. Anatoli Iambartsev - IME-USP

- Prof. Dr. Renato Mendes Coutinho - UFABC 


\section{Agradecimentos}

Esta é uma seção de extrema importância nesse trabalho. Infelizmente, poucos possuem o dom de transformar em palavras os sentimentos humanos, tentarei aqui expressar minha eterna gratidão às pessoas que possibilitaram a realização desse trabalho.

Primeiramente agradeço à Deus, pelas oportunidades concedidas e por iluminar meus caminhos.

À minha família pelo amor e o apoio incondicional à minha formação acadêmica. Em especial ao meu pai, Antonio, que sempre me serviu de inspiração e contribuiu para moldar o meu caráter.

Ao meu orientador, professor Nelson Kuhl, pelo direcionamento da pesquisa e aos valorosos conselhos, guiando-me durante a sinuosa, e por muitas vezes espinhosa, jornada de elaboração desse trabalho. À professora Claudia Peixoto pelas diversas sugestões e pela disponibilidade em resolver diversas dúvidas que surgiram no decorrer da pesquisa.

Ao pessoal do grupo de Epidemiologia do Instituto de Matemática e Estatística pelo companheirismo, amizade e inúmeras sugestões.

Agradeço ainda ao pessoal do time de beisebol e softbol do IME-USP por todo o aprendizado em relação à dedicação e persistência em busca de objetivos. Embora escassas, as horas que passamos juntos foram muito intensas e guardarei com enorme carinho todos os torneios, jogos e treinos.

Um agradecimento especial ao meu grande amigo Tiago Yoneda pela companhia e amizade desde os tempos da graduação, e por me socorrer em inúmeros apuros durante todo esse tempo.

Por fim, agradeço ao apoio da CAPES, cujo financiamento possibilita não apenas este, mas inúmeros outros trabalhos de grande relevância que contribuem para o avanço da ciência em nosso país.

À todos que direta ou indiretamente contribuíram para a realização deste trabalho, meus sinceros e profundos agradecimentos. 


\section{Resumo}

NAKASHIMA, A. T. O algoritmo de simulação estocástica para o estudo do comportamento da epidemia de dengue em sua fase inicial. 2018. 120 f. Dissertação (Mestrado) Instituto de Matemática e Estatística, Universidade de São Paulo, São Paulo, 2018.

O comportamento de sistemas epidêmicos é frequentemente descrito de maneira determinística, através do emprego de equações diferenciais ordinárias. Este trabalho visa fornecer uma visão estocástica do problema, traçando um paralelo entre o encontro entre os indivíduos em uma população e o choque entre partículas de uma reação química. Através dessa abordagem é apresentado o algoritmo de Gillespie, que fornece uma forma simples de simular a evolução de um sistema epidêmico. Fundamentos de processos estocásticos são apresentados para fundamentar uma técnica para a estimação de parâmetros através de dados reais. Apresentamos ainda o modelo de Tau-leaping e o modelo difusivo elaborado através de equações diferenciais estocásticas que são aproximações do modelo proposto por Gillespie. A aplicação dos modelos apresentados é exemplificada através do estudo de dados reais da epidemia de dengue ocorrida no estado de Rio de Janeiro entre os anos de 2012 e 2013.

Palavras-chave: modelo epidêmico, algoritmo de Gillespie, processo estocástico. 


\section{Abstract}

NAKASHIMA, A. T. The stochastic simulation algorithm for the study of the behavior of the dengue epidemic in its initial phase. 2018. $120 \mathrm{f}$. Dissertação (Mestrado) - Instituto de Matemática e Estatística, Universidade de São Paulo, São Paulo, 2018.

The behavior of epidemic systems is often described in a deterministic way, through the use of ordinary differential equations. This paper aims to provide a stochastic view of the problem, drawing a parallel between the encounter between individuals in a population and the clash between particles of a chemical reaction. Through this approach is presented the Gillespie algorithm, which provides a simple way to simulate the evolution of an epidemic system. Fundamentals of stochastic process theory are presented to support a technique for estimating parameters through real data. We present the model of emph Tau-leaping and the diffusive model elaborated by stochastic differential equations that are approximations of the model proposed by Gillespie. The application of the presented models is exemplified through the study of real data of the dengue epidemic occurred in the state of Rio de Janeiro between the years of 2012 and 2013.

Keywords: epidemic model, Gillespie algorithm, stochastic process. 


\section{Sumário}

$\begin{array}{ll}\text { Lista de Abreviaturas } & \text { ix }\end{array}$

Lista de Símbolos $\quad$ xi

Lista de Figuras $\quad$ xiii

$\begin{array}{lc}\text { Lista de Tabelas } & \text { xvii }\end{array}$

1 Introdução $\quad 1$

1.1 A organização do trabalho . . . . . . . . . . . . . . . . . . 1

2 O Modelo Matemático $\quad 3$

2.1 Sobre a Dengue . . . . . . . . . . . . . . . . . . . . 3

2.2 Dinâmica da transmissão . . . . . . . . . . . . . . . . . . . 4

2.3 Simplificações do modelo . . . . . . . . . . . . . . . . . . 4

2.4 Cinética Química . . . . . . . . . . . . . . . . . . . 5

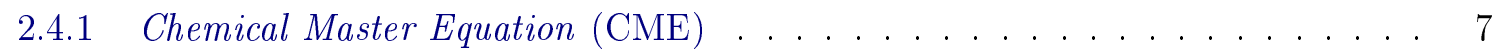

2.4 .2 O algoritmo de Gillespie $(\mathrm{SSA}) \ldots \ldots \ldots \ldots \ldots$

2.4 .3 Aplicações em epidemiologia . . . . . . . . . . . . . . . . . . . . . 9

3 Estimação de parâmetros $\quad 13$

3.1 Os dados . . . . . . . . . . . . . . . . . . . . . . . 13

3.2 Inferência através de dados completos . . . . . . . . . . . . . . . . . . . 14

3.3 Construção da base de dados discreta . . . . . . . . . . . . . . . 17

3.4 Inferência através de observações em tempo discreto . . . . . . . . . . . . . . 20

4 Resultados Obtidos $\quad 29$

4.1 Estimando parâmetros para grupos de cidades selecionados através do perfil demo-

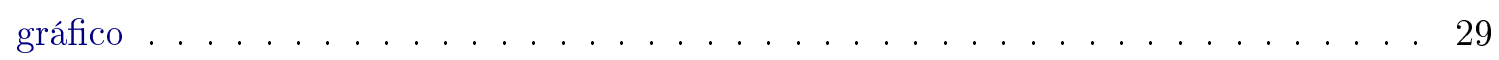

4.1.1 Grupo 1: Cidades com densidade demográfica superior à $1000 \mathrm{hab} / \mathrm{km}^{2}$. . . 29

4.1.2 Grupo 2: Cidades com densidade demográfica entre 200 e $1000 \mathrm{hab} / \mathrm{km}^{2} \ldots 34$

4.1.3 Grupo 3: Cidades com densidade demográfica abaixo de $200 \mathrm{hab} / \mathrm{km}^{2} \ldots$. . 38

4.1 .4 Testando cenários alternativos . . . . . . . . . . . . . . . . . . 44

$\begin{array}{lll}5 & \text { Aproximações por processos de difusão } & 47\end{array}$

5.1 Uma outra abordagem . . . . . . . . . . . . . . . . . . . . . 51

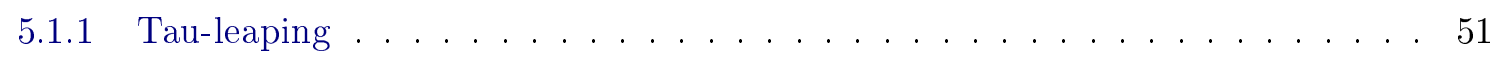


5.1 .2 A aproximação por processos de difusão . . . . . . . . . . . . . . . 52

6 Discussão $\quad 57$

6.1 Um olhar para o horizonte . . . . . . . . . . . . . . 57

A Processos Estocásticos $\quad 59$

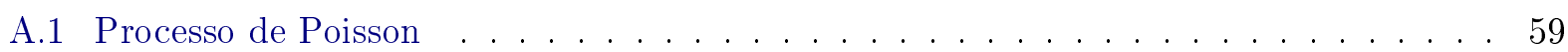

B Equações Diferenciais Estocásticas e Movimento Browniano $\quad 63$

B.1 Integrais Estocásticas . . . . . . . . . . . . . . . . . . . . 64

B.2 Método de Euler-Maruyama . . . . . . . . . . . . . . . . 64

$\begin{array}{lll}\text { C A fórmula de Taylor e a representação de derivadas } & 67\end{array}$

$\begin{array}{ll}\text { Referências Bibliográficas } & 69\end{array}$

$\begin{array}{ll}\text { Índice Remissivo } & 73\end{array}$ 


\title{
Lista de Abreviaturas
}

\author{
CME Chemical Master Equation \\ DC Dengue Clássica \\ DH Dengue Hemorrágica \\ EDO Equação Diferencial Ordinária \\ EMV Estimador de Máxima Verossimilhança \\ FCD Forma Clássica da Dengue \\ FHD Forma Hemorrágica da Dengue \\ MH Metropolis-Hastings \\ MMS Média Móvel Simples \\ SCD Síndrome de Choque da Dengue \\ SDE Stochastic Differential Equation \\ SINAN Sistema de Informação de Agravos de Notificação \\ SSA Algoritmo de Simulação Estocástica (Stochastic Simulation Algorithm)
}




\title{
Lista de Símbolos
}

\author{
$\mathbb{R}_{+}^{N} \quad$ vetor de dimensão $N$ e entradas não negativas \\ $\mathcal{R}_{j} \quad$ reação química do tipo $j$ \\ $\boldsymbol{\nu}_{j} \quad$ vetor estequiométrico associado à reação $j$ \\ $I C(\theta, y)$ intervalo de confiança para $\theta$ com nível de confiança $y$ \\ $\hat{\theta} \quad$ estimador para um parâmetro $\theta$
}




\section{Lista de Figuras}

2.1 Países com casos relatados ou risco de casos de dengue segundo a Organização Mun-

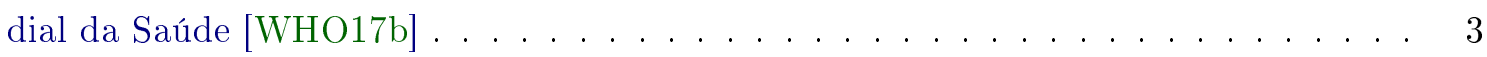

2.2 Comparação entre três sistemas homogêneos isolados com mesma densidade demográfica . . . . . . . . . . . . . . . . . . . . . . 10

3.1 Notificações de casos de dengue na cidade de Angra dos Reis no período 2012-2013 • 14

3.2 Notificações em cidades do estado do Rio de Janeiro no período 2012-2013 . . . . . . 18

3.3 Efeito da suavização por médias móveis simples no número de infectados projetados . 19

3.4 Exemplo de construção de trajetória através de um passo do método de MetropolisHastings . . . . . . . . . . . . . . . . . . . . . . . . 26

4.1 Notificações do município de Queimados. Notamos que a infecção não evolui para um regime epidêmico . . . . . . . . . . . . . . . . . . . . . . 29

4.2 Número de infectados projetados (suavizados) para a dengue por semana nas cidades do grupo 1 no período $2012-2013$. . . . . . . . . . . . . . . . . . . . . . 31

4.3 Evolução das estimativas ao longo das iterações de Metropolis-Hastings para os parâmetros para o Grupo 1. Em destaque as últimas 30 iterações do Metropolis-Hastings 31

4.4 Distribuições a priori e posteriori para os parâmetros do modelo no Grupo 1 . . . . . 32

4.5 Projeções geradas pela simulação do algoritmo de Gillespie para as cidades do grupo 1 para as semanas seguintes ao período de ajuste . . . . . . . . . . . . . . 33

4.6 Comportamento atípico no número de infectados no município de Maricá . . . . . . . 35

4.7 Número de infectados projetados (suavizados) para a dengue por semana nas cidades do grupo 2 no período $2012-2013$. . . . . . . . . . . . . . . . . . . . . 36

4.8 Estimativas para os parâmetros para o Grupo 2. Em destaque as últimas 30 iterações do Metropolis-Hastings . . . . . . . . . . . . . . . . . . . . . . . . . 37

4.9 Distribuições a priori e posteriori para os parâmetros do modelo no Grupo 2 . . . . . 37

4.10 Projeções geradas pela simulação do algoritmo de Gillespie para as cidades do grupo 2 para as semanas seguintes ao período de ajuste . . . . . . . . . . . . . 38

4.11 Número de infectados projetados (suavizados) para a dengue por semana nas cidades do grupo 3 no período $2012-2013$. . . . . . . . . . . . . . . . . . . . . . . 40

4.12 Estimativas para os parâmetros para o Grupo 3. Em destaque as últimas 30 iterações do Metropolis-Hastings . . . . . . . . . . . . . . . . . . . . . . . . . . 41

4.13 Distribuições a priori e posteriori para os parâmetros do modelo no Grupo 3 . . . . 41

4.14 Projeções geradas pela simulação do algoritmo de Gillespie para as cidades do grupo 3 para as semanas seguintes ao período de ajuste . . . . . . . . . . . . . . . 42 
4.15 Localização das cidades de cada grupo no estado do Rio de Janeiro . . . . . . . . . . 43

4.16 Projeções geradas pela simulação do algoritmo de Gillespie para diferentes valores de $p_{\text {sus }}$ para a cidade de Nova Iguaçu para as semanas seguintes ao período de ajuste 45

4.17 Projeções geradas pela simulação do algoritmo de Gillespie para diferentes valores de $p_{\text {sus }}$ para a cidade de Saquarema com período de previsão estendido . . . . . . . 46

5.1 Projeções geradas pela aproximação por processo difusivo para a cidade do Rio de Janeiro . . . . . . . . . . . . . . . . . . . . . . . 55

5.2 Projeções geradas pela aproximação por processo difusivo para a cidade de Teresópolis 56

A.1 Processo de contagem $N(t):\left\{S_{1}, S_{2}, \ldots\right\}$ representam os instantes e $\left\{X_{1}, X_{2}, \ldots\right\}$ representam os tempos intervalares entre as reações . . . . . . . . . . . . . . . . 61 


\section{Lista de Algoritmos}

1 Gillespie - Stochastic Simulation Algorithm (SSA) [Gil08] . . . . . . . . . . . . . 9

2 Processo de Poisson não homogêneo de taxa linearizada . . . . . . . . . . . . . . . 22

3 Geração de trajetória através de processo de Poisson não homogêneo de taxa linearizada 22

4 Estimação de parâmetros do modelo com observações discreta através da aproximação por Processo de Poisson não homogêneo . . . . . . . . . . . . . . . . . . 25

5 Estimação de parâmetros do modelo com observações discreta através da aproximação por Processo de Poisson não homogêneo para um conjunto de cidades com características semelhantes . . . . . . . . . . . . . . . . . 27

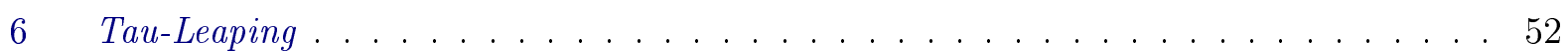

$7 \quad$ Aproximação por difusão . . . . . . . . . . . . . . . . . . . . . . 54 


\section{Lista de Tabelas}

4.1 Municípios com densidade demográfica superior a $1000 \mathrm{hab} / \mathrm{km}^{2}$ segundo o Censo

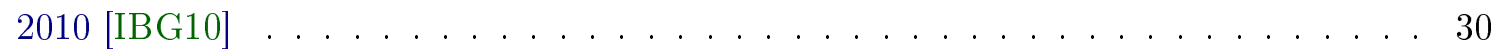

4.2 Configuração do cenário para estimação de parâmetros do grupo 1 . . . . . . . . . 30

4.3 Municípios com densidade demográfica entre 200 e $1000 \mathrm{hab} / \mathrm{km}^{2}$ segundo o Censo $2010[\mathrm{IBG} 10] \ldots \ldots \ldots \ldots \ldots \ldots$

4.4 Municípios com densidade demográfica abaixo de $200 \mathrm{hab} / \mathrm{km}^{2}$ segundo o Censo $2010[\mathrm{IBG} 10] \ldots \ldots \ldots \ldots \ldots \ldots$

4.5 Resumo dos estimadores encontrados . . . . . . . . . . . . . . . . . . 43

4.6 Resumo dos estimadores encontrados para cada valor de $\mu \ldots \ldots$. . . . . . . . 44

4.7 Resumo dos estimadores encontrados para cada valor de $p_{\text {sus }} \ldots \ldots \ldots$. . . . . . . 45 
xviii LISTA DE TABELAS 


\section{Capítulo 1}

\section{Introdução}

Embora hajam registros de doenças infecciosas que assolam a humanidade desde tempos imemoriais, ainda hoje não é muito difícil encontrar estampadas nas manchetes de jornais novos surtos epidêmicos que preocupam cientistas e causam pânico na população.

A relevância do tema pode ser destacada por trabalhos como o livro de Ujvari de 2003 [Ujv03] que, de forma muito interessante, evidencia a importância do assunto, traçando a história da humanidade através das epidemias que se abateram sobre ela, exibindo diversos episódios em que a negligência em relação às doenças infecciosas ocasionaram em resultados catastróficos, derrubando impérios e decidindo guerras.

Mais recentemente observamos eclodirem epidemias de Ebola em 2014, inicialmente identificada em 23 de março desse ano e que, agravada pelo precário sistema sanitário e de saúde existentes na região [BFJ14] já totalizava 4507 casos prováveis e confirmados, com 2296 mortes reportadas em 14 de setembro ao longo de cinco países da África Ocidental: República da Guiné, Libéria, Nigéria, Senegal e Serra Leoa [Tea14].

No Brasil, as principais epidemias enfrentadas nos últimos anos estão associadas às arboviroses: Dengue, Chikungunya e Zika, sendo que à essa última infecção é estudada uma relação com os casos de microcefalia registrados em 2015 [SF16]. Já entre o final de 2016 e os primeiros meses de 2017, foi registrado um grande surto de febre amarela no Brasil, em regiões antes classificadas como livres de contágio [RSO $\left.{ }^{+} 17\right]$, levando as autoridades a adotar campanhas de prevenção com o fechamento de áreas em que foram verificadas a mortalidade de macacos (hospedeiro do vírus na mata) e uma extensa campanha de imunização através da vacina.

Diante desse cenário, pesquisadores das mais diversas áreas debruçaram-se sobre o tema na tentativa de compreender melhor os meios em que se dão a transmissão e a disseminação de doenças.

O objetivo do presente trabalho é propor uma abordagem matemática ao tema, através de um modelo simplificado que trata as incertezas inerentes do problema através de mecanismos estocásticos.

A dengue foi escolhida como doença alvo do estudo devido aos surtos dessa moléstia que tem sido recorrentes em boa parte do país na última década. Corroborou na nossa escolha o fato de ser possível obter informações sobre o histórico recente da doença através do Sistema de Informação de Agravos de Notificação (SINAN) [SIN16], base de dados ligado ao Ministério da Saúde do Brasil.

\subsection{A organização do trabalho}

Apresentaremos agora um esquema básico que sustenta a construção do trabalho. O capítulo 1 tem caráter meramente introdutório e tem por finalidade ambientar o leitor ao tema discutido na pesquisa.

O início do capítulo 2 contém um resumo básico com características relevantes da dengue, exibindo as simplificações adotadas na modelagem do problema. Logo em seguida o leitor é familiarizado a alguns conceitos da teoria de química cinética e a dedução do algoritmo de Gillespie. Durante 
esse trajeto são discutidas algumas ideias que justificam a abordagem do problema epidemiológico através de um modelo de reações químicas.

O foco do capítulo 3 é prover uma ferramenta para a estimação de parâmetros através de dados reais. Para tanto, é discutida a natureza dos dados disponíveis para a análise, destacando os ajustes necessários antes de sua utilização. Apresentamos um caso idealizado, exibindo a dedução de estimadores a partir de dados contínuos no tempo e depois discutimos o caso real em que é necessário um estimador a partir de observações discretas.

Os resultados da utilização das metodologias de estimação e simulação estocástica de sistemas epidemiológicos são apresentadas no capítulo 4. Aqui são selecionadas cidades do estado do Rio de Janeiro com registro de surtos epidêmicos ocorridos no período de 2012 a 2013. Diversos gráficos são apresentados com o intuito de projetar o comportamento do sistema nas semanas seguintes ao período base, utilizado para estimar parâmetros (previsão de futuro próximo).

No capítulo 5, por sua vez, são apresentadas métodos estocásticos alternativos, que aproximam o algoritmo de Gillespie. Tais métodos podem ser úteis em cidades populosas, em que o modelo de Gillespie se mostra oneroso computacionalmente.

Por fim, no capítulo 6 é elaborada uma retrospectiva dos principais pontos discutidos ao longo do trabalho e abordamos possíveis linhas de pesquisa futura.

Durante o processo de composição desse trabalho, foram requeridos fundamentos teóricos de diferentes áreas do conhecimento. Com a finalidade de favorecer o entendimento da pesquisa foram anexados textos introdutórios que apresentam princípios básicos de determinada teoria, requisitada ao longo do desenvolvimento do texto. O primeiro dos anexos, que se encontram ao final do trabalho, consiste em um texto básico dos processos estocásticos, com um enfoque no processo conhecido processo de Poisson. No segundo texto, apresentamos uma introdução às equações diferenciais estocásticas e o movimento Browniano. Já no último anexo, são apresentadas as representações de derivadas a partir do truncamento de expansões em séries de Taylor.

Os textos anexados podem não ser suficientes ao leitor mais ávido ao aprofundamento nas respectivas áreas, porém acreditamos que elas podem tornar a experiência de leitura dessa obra mais enriquecedora.

Este trabalho, juntamente com os códigos elaborados durante a pesquisa, escritas em linguagem R, serão disponibilizados no endereço https://www.ime.usp.br/ naka/pos. 


\section{Capítulo 2}

\section{O Modelo Matemático}

\subsection{Sobre a Dengue}

A dengue é um dos principais problemas de saúde pública no mundo. Causada por um vírus do gênero flavivírus, da família flaviviridae, apresenta-se em quatro sorotipos atualmente conhecidos: $D E N V 1, D E N V 2, D E N V 3$ e DENV4 [RMVC06].

Trata-se de uma doença que ocorre em regiões tropicais e subtropicais, como pode ser verificado na figura 2.1, predominantemente em áreas urbanas e semi-urbanas.

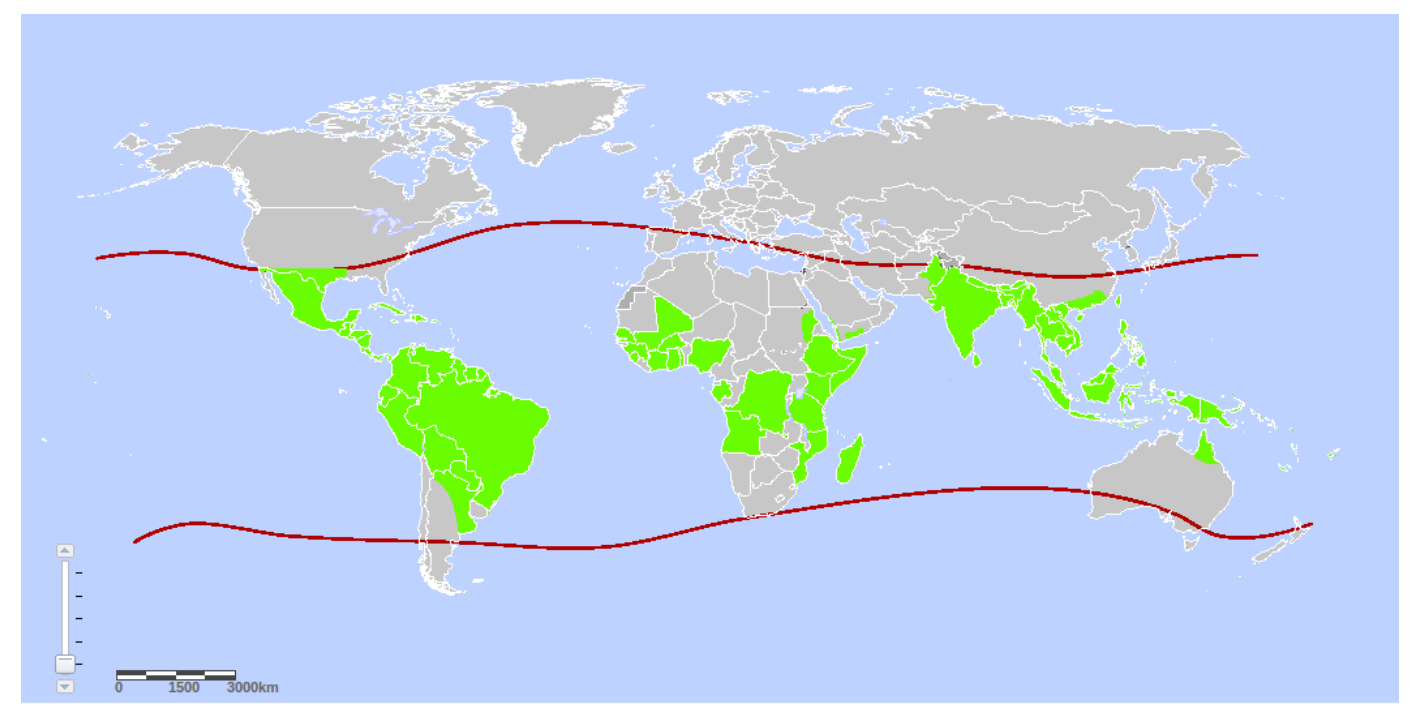

Figura 2.1: Países com casos relatados ou risco de casos de dengue segundo a Organização Mundial da Saúde [WHO1'b]

A dengue é uma doença transmitida por mosquitos do gênero Aedes. Embora o Aedes albopictus já seja estudado como vetor secundário para a dengue na Ásia [Tau01], e já hajam relatos desse mosquito naturalmente infectados no México [IBBM ${ }^{+}$97], aqui no Brasil consideramos a dengue transmitida exclusivamente pelo Aedes aegypti.

No Brasil, o Aedes aegypti, mosquito encontrado principalmente no meio urbano, chegou a ser declarado erradicado em 1955 em um esforço conjunto dos países americanos, porém foi reintroduzido no país em 1976 em funções de falhas na vigilância epidemiológica e das mudanças demográficas e sociais provocadas pela urbanização no período [BV07].

A intensa migração rural-urbana presenciada sobretudo a partir da década de 60 com o crescimento desordenado dos grandes centros urbanos, que não conseguiram atender às demandas básicas de habitação e saneamento básico para essa nova população, facilitaram a proliferação do vetor e são apontadas como possíveis das causas do avanço da doença durante o período [BV07]. Estima-se que entre $20 \%$ e $25 \%$ dessa população ainda vivam em favelas, invasões e cortiços [Tau01]. 
Outro fator que contribuiu para facilitar a propagação do vírus da dengue é o significativo aumento da intensidade e da velocidade do tráfego aéreo e terrestre. Por meio do sangue das pessoas portadoras da infecção, em poucos dias o agente infeccioso pode percorrer distâncias continentais [Tau01].

\subsection{Dinâmica da transmissão}

A dengue possui apenas um ciclo epidemiológico cujos componentes são o homem (hospedeiro) e o mosquito (vetor). A fêmea suscetível do mosquito infecta-se com o vírus da dengue ao alimentar-se do sangue de um humano em estado infectante, período em que o indivíduo está infectado e está apto a transmitir a infecção. O mosquito, por sua vez, mantém-se infectante pelo restante de sua vida. Já um indivíduo suscetível adquire a infecção através da picada de um vetor infectante, podendo evoluir para uma forma clássica (DC) com a ocorrência de febre, mialgias e artralgias ou para uma forma mais grave, conhecida como dengue hemorrágica $(\mathrm{DH})$, caracterizada por distúrbios de coagulação. Os casos mais graves de dengue hemorrágica podem evoluir para a síndrome de choque da dengue (SCD) com hemoconcentração e falência circulatória podendo ocasionar o óbito do indivíduo em 12 ou 24 horas ou à recuperação após a terapia anti-choque [Yan03, PTP98].

Não há imunidade cruzada, ou seja, a infecção por um sorotipo confere apenas imunidade permanente, ou ao menos duradoura àquele sorotipo. Há a possibilidade da existência de imunidade cruzada transitória, de curta duração, entre os sorotipos [Tau01].

É aceito que uma primeira infecção por qualquer sorotipo leva o indivíduo a adquirir a forma clássica da dengue (FCD). Por outro lado, a reinfecção por um outro antigênico do vírus da dengue desencadearia a forma hemorrágica da doença (FHD). Admite-se ainda que qualquer dos sorotipos pode provocar a forma hemorrágica da dengue, entretanto estudos clínicos evidenciaram uma correlação entre o número de casos de dengue hemorrágica e o sorotipo DENV2, quando este ocorria como infecção secundária. Há, todavia, relatos de epidemias da FHD, causadas pelo sorotipo DENV3, comprovando que as relações entre infecções primárias e secundárias, suas combinações e consequências estão ainda longe de ser totalmente esclarecidas [Ver91, Yan03].

Segundo a Organização Mundial da Saúde (World Health Organization) [WHO17a], o vírus da dengue circula no sangue da pessoa infectada por 2 a 7 dias, período em que o paciente desenvolve febre. Após o surgimento dos primeiros sintomas, o indivíduo permanece infeccioso, ou seja, com potencial de transmissão por um período de 4 até no máximo 12 dias.

\subsection{Simplificações do modelo}

Conforme visto na seção 2.2, a transmissão da dengue de um indivíduo infectado para um suscetível é intermediada pela fêmea de um mosquito Aedes aegypti, que é denominada vetor da doença. Portanto, para descrever a dinâmica da infecção seria necessário obter alguns parâmetros acerca das características dos mosquitos como, por exemplo, o número de mosquitos em uma determinada região e, embora hajam iniciativas que buscam através da contagem de ovos, larvas ou até mesmo do mosquitos adultos em armadilhas para estimar a população total [ $\left.\mathrm{BGN}^{+} 00, \mathrm{dFBdCC}^{+} 01\right]$, tais procedimentos ainda não foram suficientemente difundidas para obtermos dados confiáveis.

Optamos então por simplificar a dinâmica de transmissão da dengue, eliminando o vetor da doença. Dessa forma, consideramos que um indivíduo infectado transmite diretamente a doença à um suscetível, sem intermédio de um mosquito. Felizmente, como o alcance de voo da fêmea do Aedes aegypti é reduzido (em geral, menos de $1 \mathrm{~km}$ ) [MB98, TH86], podemos atribuir à movimentação dos humanos como responsável pela disseminação da dengue entre as cidades.

Outra dificuldade enfrentada no estudo do modelo de dengue no Brasil é o fato de que, em muitos casos, durante um surto epidêmico, o teste sorológico não é efetuado, tornando impossível a identificação do sorotipo que desencadeou o processo infeccioso no paciente.

Diante desse cenário, optamos por utilizar um modelo simplificado, sem discriminação de sorotipo. Para tanto, algumas hipóteses foram consideradas: 
Hipótese 2.3.1. Simplificações do modelo proposto

(a) Em um surto epidêmico, consideramos ativo apenas um único sorotipo da doença;

(b) No período de um ano, apenas um surto epidêmico ocorre. Um indivíduo pode infectar-se, no máximo, uma vez ao ano;

(c) O modelo exibe a dinâmica de um surto epidêmico;

(d) Antes da notificação dos primeiros casos da doença, toda a população da cidade estudada é considerada suscetivel;

A dengue é uma patologia cuja notificação é compulsória [PTP98], ou seja, sempre que um indivíduo e diagnosticado com dengue, seja ela FCD ou FHD, é dever da unidade de atendimento informar a secretaria de saúde. Este rígido controle, no entanto, não impede que o número de notificações de casos de dengue subestime o número de infectados. Isso ocorre devido ao fato de que uma parcela dos infectados na FCD, que denominaremos infectados assintomáticos, apresentam sintomas mais brandos, e seus diagnósticos muitas vezes confundidos com outras doenças, como a gripe.

Trabalhos como os publicados em 2013 por Bhatt et al. [BGB $\left.{ }^{+} 13\right]$ e García et al. de 2010 [GSP $\left.{ }^{+} 10\right]$ ajudam a mostrar que o papel dos infectados assintomáticos na dinâmica da epidemia não pode ser ignorado. A relevância dos assintomáticos foi evidenciada também no trabalho de Seed et al. 2009 [SKHK09], dessa vez com o foco nas transmissões através de transfusões de sangue na Austrália.

Na tentativa de estimar o número de infectados assintomáticos, proporemos uma hipótese adicional:

Hipótese 2.3.2. Infectados assintomáticos

Denotando por $I_{s}$ o número de infectados sintomáticos, ou seja, aqueles são atendidos em uma unidade de saúde e geram uma notificação de dengue, e $I_{a}$ o número de infectados assintomáticos, existe $\mu>0$ tal que:

$$
\left\{\begin{array}{l}
I_{a}=\mu I_{s} \\
I=I_{a}+I_{s}
\end{array}\right.
$$

onde I representa o número total de infectados.

Consideramos ainda que a dinâmica de transmissão e recuperação de um infectado assintomático se dá nos mesmos moldes do que ocorre com um infectado sintomático.

Vamos ainda salientar um outro fato relevante: quando verifica-se uma rápida disseminação de uma doença é esperado de hajam intervenções externas tanto da população que se encontra em risco, quanto das autoridades sanitárias, de saúde e do governo, alterando a dinâmica da doença. Especificamente no caso da dengue, há ainda a sensibilidade da doença em relação ao regime de chuvas da região.

Por esses motivos apresentados, não espera-se que o modelo proposto ajuste de forma razoável a dinâmica da doença após um período. Vamos então focar nossos esforços na tentativa de capturar de forma satisfatória o comportamento da doença no início da disseminação da epidemia, que aqui fixaremos entre 4 a 5 semanas. Referiremos-nos à esse intervalo de tempo como período de ajuste do modelo.

\subsection{Cinética Química}

Traçaremos agora um paralelo entre a dinâmica de transmissão de doenças e as reações químicas elementares, justificando nossa abordagem de modelos epidemiológicos através da teoria de cinética 
química. As definições aqui descritas e a notação empregada derivam principalmente dos trabalhos de Gillespie [Gi108], Higham [Hig08], de Gupta [Gup13] e do livro de Wilkinson [Wil11].

Uma reação é dita elementar quando uma ou mais espécies químicas envolvidas reagem diretamente formando produtos em uma única etapa [IUP17].

Considere um processo químico envolvendo $N$ diferentes tipos de moléculas ou espécies químicas $\left\{E_{1}, E_{2}, \ldots, E_{N}\right\}$, envolvidas em $M$ reações distintas $\left\{\mathcal{R}_{1}, \mathcal{R}_{2}, \ldots, \mathcal{R}_{M}\right\}$.

Considerando as leis da física, se conhecermos a posição e velocidade de todas as partículas de cada uma das espécies, traçando suas trajetórias, verificando as colisões entre as moléculas e considerando suas respectivas resultantes, somos capazes de descrever o estado do sistema em qualquer instante de tempo [Hig08]. Entretanto, uma abordagem altamente detalhada como esta requer um elevado custo computacional, o que a torna inviável para quase todo sistema real.

Em sistemas em que as moléculas estão espalhadas uniformemente por todo o seu domínio, denotado aqui por $\Omega$, podemos utilizar uma simplificação, ignorando a informação espacial e detalhando apenas o número de moléculas de cada espécie [Hig08].

O estado do sistema em um instante $t>0$ é uma variável aleatória vetorial, denotada por $\mathbf{X}(t) \in \mathbb{R}_{+}^{N}$. Ou seja:

$$
\mathbf{X}(t)=\left[\begin{array}{c}
X_{1}(t) \\
X_{2}(t) \\
\vdots \\
X_{N}(t)
\end{array}\right]
$$

onde $X_{i}(t)$ representa uma variável aleatória escalar que representa o número de moléculas em $\Omega$ da espécie química $E_{i}$ no instante $t$ [Gup13]. Nesse trabalho, uma amostra $\mathbf{x}$ da variável aleatória $\mathbf{X}(t)$ será denotada por:

$$
\mathbf{x}=\left[\begin{array}{c}
x_{1} \\
x_{2} \\
\vdots \\
x_{N}
\end{array}\right]
$$

onde $x_{i}$ representa uma amostra para a variável aleatória $X_{i}(t)$, para $i=1, \ldots, N$.

Para sistemas bem agitados (Well-stirred systems), para cada tipo de reação $\mathcal{R}_{j}$, definimos $\boldsymbol{\nu}_{j}=\left(\nu_{1 j}, \ldots, \nu_{N j}\right)$ o vetor de troca de estados ou vetor estequiométrico, onde $\nu_{i j}$ representa a variação no número de moléculas da $i$-ésima espécie ocasionada pelo evento $\mathcal{R}_{j}$. Então, se em um intervalo $[t, t+\Delta t]$ ocorre uma reação $\mathcal{R}_{j}$, o vetor de estados passará de $\mathbf{X}(t)=\mathbf{x}$ para $\mathbf{X}(t+\Delta t)=\mathbf{x}+\boldsymbol{\nu}_{j}$

Além disso, associada à cada reação $\mathcal{R}_{j}$, dada uma constante $\theta_{j}$, definimos a função de propensidade $a_{j}\left(\mathbf{x}, \theta_{j}\right)$, chamada também de função hazard [Wil11], que possui a seguinte propriedade: a probabilidade da reação $\mathcal{R}_{j}$ ocorrer no intervalo infinitesimal $[t, t+\Delta t]$ é dado por $a_{j}\left(\mathbf{x}, \theta_{j}\right) \Delta t$. Ou seja, se $\mathbf{X}(t)=\mathbf{x}$ :

$$
P\left[\mathbf{X}(t+\Delta t)=\mathbf{x}+\boldsymbol{\nu}_{j} \mid \mathbf{X}(t)=\mathbf{x}\right]=a_{j}\left(\mathbf{x}, \theta_{j}\right) \Delta t
$$

Notemos que na expressão 2.3 o estado do sistema em $t+\Delta t$ independe de conhecimentos anteriores ao instante $t$, de forma que a propriedade Markoviana (definida no anexo A) é satisfeita e, de fato, $\mathbf{X}$ é um processo Markoviano em tempo contínuo.

Uma reação elementar pode ser classificada conforme sua molecularidade, isto é, com relação ao número de moléculas colidindo envolvidas em uma única etapa de reação. Chamamos de unimolecular, as reações envolvendo uma única entidade molecular. No caso de duas entidades moleculares, 
a reação é classificada como bimolecular. Chamamos as reações com três entidades envolvidas de termolecular. Não consideraremos as reações termoleculares devido à improbabilidade de três moléculas colidirem simultaneamente [IUP17].

Para que reação bimolecular $\mathcal{R}_{j}$ entre duas espécies químicas distintas, $E_{m}$ e $E_{n}$ ocorra, é necessário a colisão entre uma molécula de $E_{m}$ e uma de $E_{n}$. Esse encontro é proporcional ao número de moléculas de cada uma dessas espécies. Além disso, como nem toda colisão ocasiona uma reação, então a constante $\theta_{j}$ é empregada de tal forma que $\theta_{j} x_{m} x_{n} d t$ descreva a probabilidade da reação $\mathcal{R}_{j}$ ocorrer no intervalo $[t, t+d t]$ [Hig08].

Logo, para este caso a função de propensidade é dada por: $a_{j}\left(\mathbf{x}, \theta_{j}\right)=\theta_{j} x_{m} x_{n}$.

Um raciocínio análogo pode ser empregado nos demais tipos de reações elementares. Podemos então descrever as reações elementares com suas respectivas funções de propensidade [Hig08]:

(1) Unimoleculares (Primeira Ordem): Uma molécula de uma espécie química se transforma em outra:

$$
\begin{array}{rll}
E_{m} & \stackrel{\theta_{j}}{\longrightarrow} \text { produto } \\
a_{j}\left(\mathbf{x}, \theta_{j}\right) & =\theta_{j} x_{m}
\end{array}
$$

(2) Bimoleculares(Segunda Ordem): Uma molécula de uma substância $E_{m}$ e outra da substância $E_{n}$ :

$$
\begin{aligned}
E_{m}+E_{n} & \stackrel{\theta_{j}}{\longrightarrow} \text { produto com } m \neq n \\
a_{j}\left(\mathbf{x}, \theta_{j}\right) & =\theta_{j} x_{m} x_{n}
\end{aligned}
$$

(3) Bimoleculares (Dimerização): Duas moléculas de uma mesma substância:

$$
\begin{aligned}
E_{m}+E_{m} & \stackrel{\theta_{j}}{\rightarrow} \text { produto } \\
a_{j}\left(\mathbf{x}, \theta_{j}\right) & =\frac{1}{2} \theta_{j} x_{m}\left(x_{m}-1\right)
\end{aligned}
$$

Definição 2.4.1. Propensidade separável [Gup13]

Uma função de propensidade $a_{j}\left(\mathbf{x}, \theta_{j}\right)$ é dita separável se a parte paramétrica $\theta_{j}$ e o estado do sistema podem ser separados na forma:

$$
a_{j}\left(\mathbf{x}, \theta_{j}\right)=\theta_{j}^{c_{j}} g_{j}(\mathbf{x})
$$

onde $c_{j}$ é uma constante conhecida.

\subsubsection{Chemical Master Equation (CME)}

Vamos agora definir $P\left(\mathbf{X}(t)=\mathbf{x} \mid \mathbf{X}\left(t_{0}\right)=\mathbf{x}_{\mathbf{0}}\right)$ como sendo a probabilidade do sistema se apresentar no estado $\mathbf{X}(t)=\mathbf{x}$ dado um estado $\mathbf{x}_{0}$ no instante inicial $t_{0}$.

Considerando a evolução temporal, algumas propriedades de probabilidade e da definição da função de propensidade, podemos calcular a probabilidade do sistema se encontrar no estado x no instante $t+d t$ [Gil08]:

$$
\begin{aligned}
P\left(\mathbf{X}(t+d t)=\mathbf{x} \mid \mathbf{X}\left(t_{0}\right)=\mathbf{x}_{0}\right) & =P\left(\mathbf{X}(t)=\mathbf{x} \mid \mathbf{X}\left(t_{0}\right)=\mathbf{x}_{0}\right)\left[1-\sum_{j=1}^{M}\left(a_{j}\left(\mathbf{x}, \theta_{j}\right)\right) d t\right]+ \\
& +\sum_{j=1}^{M} P\left(\mathbf{X}(t)=\mathbf{x}-\boldsymbol{\nu}_{j} \mid \mathbf{X}\left(t_{0}\right)=\mathbf{x}_{0}\right)\left[a_{j}\left(\mathbf{x}-\boldsymbol{\nu}_{j}, \theta_{j}\right) d t\right]
\end{aligned}
$$


O primeiro termo do lado direito da igualdade 2.4 representa a probabilidade do sistema estar no estado $\mathbf{x}$ no instante $t$ e não ocorrer nenhuma mudança após decorrido um tempo $d t$. Já o segundo termo representa a probabilidade da mudança de estado de $\mathbf{x}_{0}$ a $\mathbf{x}$ ter ocorrido em outro instante.

Subtraindo $P\left(\mathbf{X}(t)=\mathbf{x} \mid \mathbf{X}\left(t_{0}\right)=\mathbf{x}_{0}\right)$ de ambos os lados da expressão 2.4, dividindo por $d t$ e tomando o seu limite a zero $(d t \rightarrow 0)$, obtemos a expressão da equação mestra ou Chemical Master Equation (CME):

$$
\begin{aligned}
\frac{\partial P\left(\mathbf{X}(t)=\mathbf{x} \mid \mathbf{X}\left(t_{0}\right)=\mathbf{x}_{0}\right)}{\partial t} & =\sum_{j=1}^{M}\left[a_{j}\left(\mathbf{x}-\boldsymbol{\nu}_{j}, \theta_{j}\right) P\left(\mathbf{X}(t)=\mathbf{x}-\boldsymbol{\nu}_{j} \mid \mathbf{X}\left(t_{0}\right)=\mathbf{x}_{0}\right)+\right. \\
& \left.-a_{j}\left(\mathbf{x}, \theta_{j}\right) P\left(\mathbf{X}(t)=\mathbf{x} \mid \mathbf{X}\left(t_{0}\right)=\mathbf{x}_{0}\right)\right]
\end{aligned}
$$

Notemos que o conjunto discreto dos valores que $\mathbf{x}$ pode assumir é bem vasto, e o CME 2.5 é um sistema de equações diferenciais ordinárias linear com uma equação para cada estado possível de $\mathbf{x}$ [Hig08].

\subsubsection{O algoritmo de Gillespie (SSA)}

O alto número de equações envolvidas no CME torna sua simulação computacional inviável [Hig08]. Uma alternativa é a construção de realizações numéricas de $\mathbf{X}(t)$, i.e., simulando trajetórias de $\{\mathbf{X}(t), t\}$. A ideia é reunir informações sobre o sistema através da observação de várias realizações dessas trajetórias.

Vamos considerar o cenário em que temos $\mathbf{X}(t)=\mathbf{x}$ e nenhuma reação de qualquer tipo ocorra durante um intervalo de tempo. Usando a função de propensidade, temos válida a seguinte relação:

$$
P(\mathbf{X}(t+\tau+d \tau)=\mathbf{x} \mid \mathbf{X}(t)=\mathbf{x})=P(\mathbf{X}(t+\tau)=\mathbf{x} \mid \mathbf{X}(t)=\mathbf{x})\left(1-\sum_{k=1}^{M} a_{k}\left(\mathbf{x}, \theta_{k}\right) d \tau\right)
$$

Definindo $\boldsymbol{\theta}=\left(\theta_{1}, \ldots, \theta_{M}\right)$, seja $a_{0}(\mathbf{x}, \boldsymbol{\theta})=\sum_{k=1}^{M} a_{k}\left(\mathbf{x}, \theta_{k}\right)$. Subtraindo $P(\mathbf{X}(t+\tau)=\mathbf{x} \mid \mathbf{X}(t)=$ $\mathbf{x})$ e dividindo por $d \tau$ em ambos os lados de 2.6, e tomando o limite $d \tau \rightarrow 0$, temos:

$$
\frac{d P(\mathbf{X}(t+\tau)=\mathbf{x} \mid \mathbf{X}(t)=\mathbf{x})}{d \tau}=-a_{0}(\mathbf{x}, \boldsymbol{\theta}) P(\mathbf{X}(t+\tau)=\mathbf{x} \mid \mathbf{X}(t)=\mathbf{x})
$$

Com a condição inicial $P(\mathbf{X}(t+0) \mid \mathbf{X}(t)=\mathbf{x})=1$, temos que a solução da EDO 2.7 é dada por:

$$
P(\mathbf{X}(t+\tau)=\mathbf{x} \mid \mathbf{X}(t)=\mathbf{x})=e^{-a_{0}(\mathbf{x}, \boldsymbol{\theta}) \tau}
$$

Definimos agora a função $p(\tau, j \mid \mathbf{x}, t)$ tal que $p(\tau, j \mid \mathbf{x}, t) d \tau$ represente a probabilidade de, dado $\mathbf{X}(t)=\mathbf{x}$ a próxima reação no sistema ocorra no intervalo infinitesimal $[t+\tau, t+\tau+d \tau]$ e seja a reação $\mathcal{R}_{j}$. Usando as definições de $a_{j}$, obtemos:

$$
p(\tau, j \mid \mathbf{x}, t) d \tau=P(\mathbf{X}(t+\tau)=\mathbf{x} \mid \mathbf{X}(t)=\mathbf{x}) a_{j}\left(\mathbf{x}, \theta_{j}\right) d \tau
$$

Daí segue que:

$$
p(\tau, j \mid \mathbf{x}, t)=a_{j}\left(\mathbf{x}, \theta_{j}\right) e^{-a_{0}(\mathbf{x}, \boldsymbol{\theta}) \tau}=\frac{a_{j}\left(\mathbf{x}, \theta_{j}\right)}{a_{0}(\mathbf{x}, \boldsymbol{\theta})} a_{0}(\mathbf{x}, \boldsymbol{\theta}) e^{-a_{0}(\mathbf{x}, \boldsymbol{\theta}) \tau}
$$

Isso mostra que o tempo até a próxima reação, $\tau$, é uma variável aleatória com distribuição 
exponencial com média (e desvio padrão) $\frac{1}{a_{0}(\mathbf{x}, \boldsymbol{\theta})}$ e o índice da próxima reação, $j$, é uma variável aleatória inteira com probabilidade dada por $\frac{a_{j}\left(\mathbf{x}, \theta_{j}\right)}{a_{0}(\mathbf{x}, \boldsymbol{\theta})}$ [Gil08]. Notemos que $\tau$ e $j$ são independentes, o que nos permite simular independentemente o índice da reação $(j)$ e o tempo da reação $(\tau)$. $p(\tau, j \mid \mathbf{x}, t)$ é então, a função densidade conjunta de suas variáveis aleatórias [Hig08].

Além disso, a evolução do número de reações de um determinado tipo $\mathcal{R}_{j}$ define um processo não homogêneo de Poisson de intensidade dada por $a_{j}\left(\mathbf{x}, \theta_{j}\right)$.

Então dado um estado inicial $\mathbf{X}\left(t_{0}\right)=\mathbf{x}_{\mathbf{0}}$ podemos gerar uma sequência de pares ordenados $\left\{\left(t_{i}, \mathbf{x}\left(t_{i}\right)\right)\right\}$ em que o primeiro elemento, $t_{i}$, indica o instante em que a $i$-ésima reação ocorre e $\mathbf{x}\left(t_{i}\right)$ indica o novo estado do sistema.

Podemos então, apresentar um pseudocódigo que nos permite simular nosso sistema ao longo do tempo.

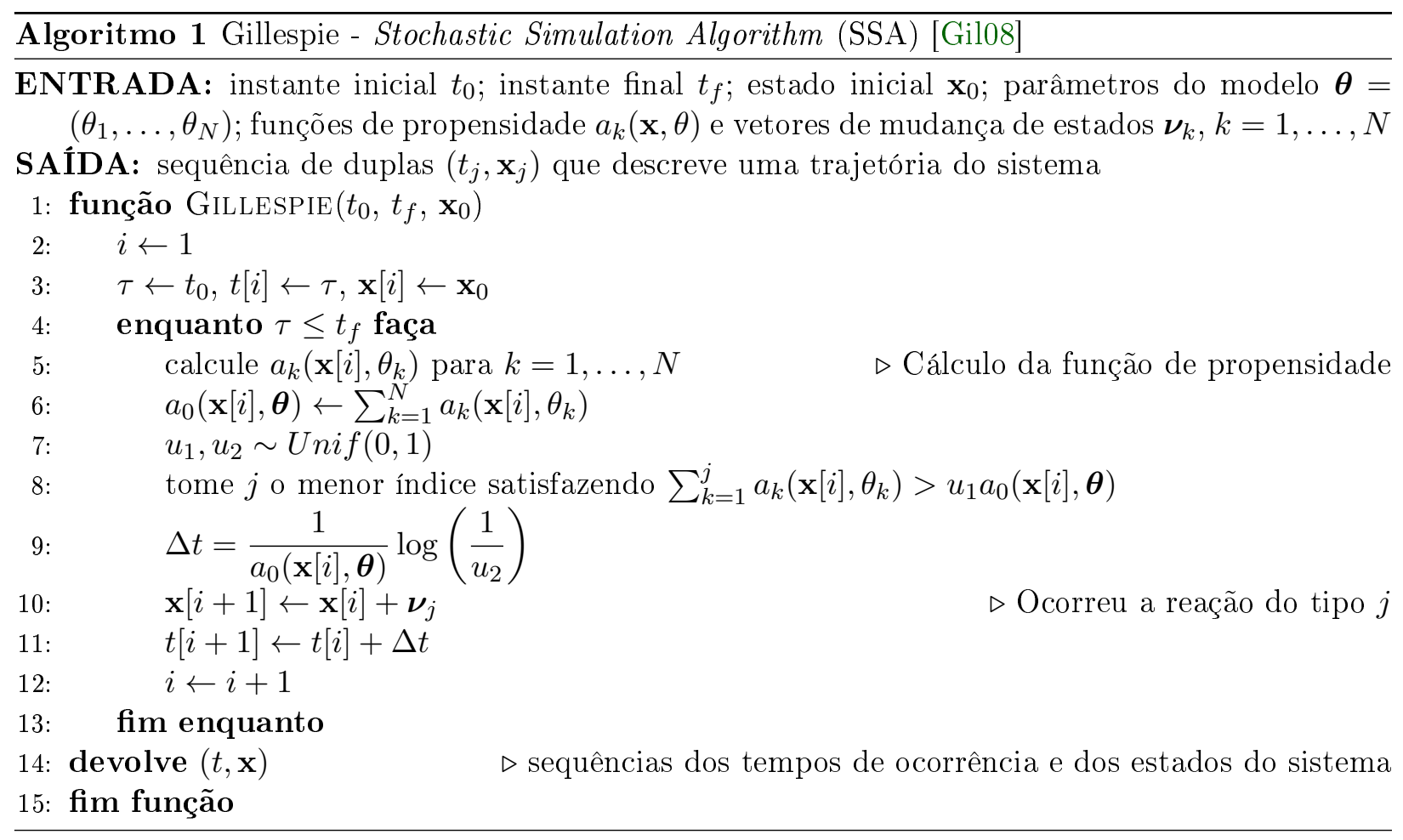

Notemos que a variável tempo até a próxima reação, $\tau$, foi gerada utilizando uma variável aleatória com distribuição Uniforme através de uma técnica conhecida como método da transformação inversa para geração de variável aleatória, cujos detalhes podem ser conferidos no livro de Florescu de 2014 [Flo14].

\subsubsection{Aplicações em epidemiologia}

O modelo proposto na seção 2.4 é válido para sistemas bem agitados (Well-stirred systems) . Analogamente, para utilizarmos esse modelo em problemas envolvendo epidemias precisamos supor a homogeneidade da população no sentido de que a probabilidade de um dado indivíduo encontrar qualquer outro é sempre igual.

Vamos dividir a população em três grupos: suscetíveis $(\mathrm{S})$, infectados (I) e recuperados $(\mathrm{R})$, que representarão as espécies químicas do sistema. Consideramos nesse modelo que todo indivíduo infectado é também infectivo, e portanto, possui potencial de transmitir a doença. Para esse sistema, as seguintes reações são consideradas: 


$$
\begin{array}{ll}
\mathcal{R}_{1}: & S+I \stackrel{\alpha}{\rightarrow} 2 I \\
\mathcal{R}_{2}: & I \stackrel{\gamma}{\rightarrow} R
\end{array}
$$

$\mathcal{R}_{1}$ representa a infecção de um indivíduo através do encontro e contato com um indivíduo infectado. Já $\mathcal{R}_{2}$ representa a recuperação de um indivíduo infectado. Temos então $\boldsymbol{\theta}=\left(\theta_{1}, \theta_{2}\right)=$ $(\alpha, \gamma)$.

Dado um estado $\mathbf{x}(t)=(S(t), I(t), R(t))$, temos associada a cada reação uma função de propensidade:

$$
\begin{aligned}
a_{1}(\mathbf{x}(t), \alpha) & =\alpha S(t) I(t) \\
a_{2}(\mathbf{x}(t), \gamma) & =\gamma I(t)
\end{aligned}
$$

Até agora estivemos preocupados em estudar o comportamento de um único sistema em um meio. No nosso caso, estamos utilizando a hipótese de que a mesma doença provoca os surtos epidêmicos de cada cidade (hipótese 2.3.1). É então natural que as medidas paramétricas entre as cidades sejam comparáveis.

Para esclarecer esse ponto, considere o seguinte exemplo: Suponha que temos três sistemas homogêneos isolados, com densidade demográfica semelhante, cuja composição é descrita abaixo:

Sistema 1: 4 indivíduos suscetíveis, 2 infectados e 2 recuperados;

Sistema 2: 4 indivíduos suscetíveis, 2 infectados e 5 recuperados;

Sistema 3: 8 indivíduos suscetíveis, 4 infectados e 4 recuperados,

onde densidade demográfica ou população relativa é definida como sendo o número de indivíduos de uma determinada região dividido pela sua área territorial.

A figura 2.2 exibe graficamente o exemplo.

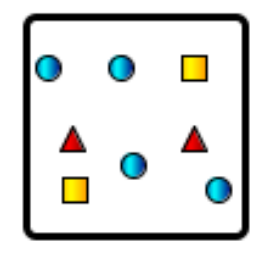

(1)

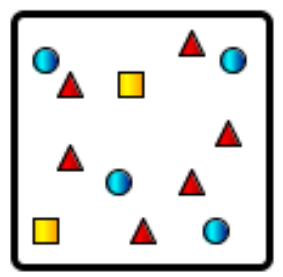

(2)

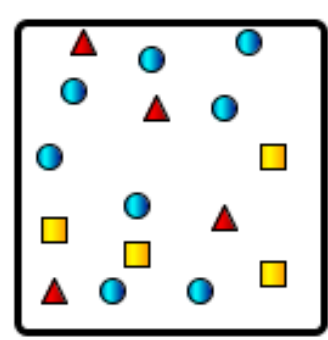

(3)

Figura 2.2: Comparação entre três sistemas homogêneos isolados com mesma densidade demográfica

Desejamos que o parâmetro $\alpha$ seja uma medida de nível de infecciosidade da doença. Como a doença é a mesma para os três sistemas do exemplo, desejamos que $\alpha$ seja parametricamente comparável entre eles. Em outras palavras, para cidades com densidade demográfica semelhante e afligidas pela mesma doença infecciosa, espera-se que o parâmetro de infecção $\alpha$ seja próximo.

Para a reação bimolecular ocorrer é necessário que haja a colisão entre duas partículas. Traduzindo esse fato para o nosso caso, para que uma infecção (reação $\mathcal{R}_{1}$ ) ocorra, é necessário que haja o contato entre um indivíduo suscetível com um infectado. A função responsável por quantificar a probabilidade de encontro entre os elementos envolvidos na reação é a função de propensidade $a_{1}()$.

Considerando $\alpha_{1}, \alpha_{2}$ e $\alpha_{3}$ os parâmetros de infecciosidade dos sistemas 1,2 e 3 , respectivamente, podemos calcular a função de propensidade para a reação de infecção de acordo com a expressão 2.11: 


$$
\left\{\begin{array}{l}
a_{1}^{1}\left(\mathbf{x}(t), \alpha_{1}\right)=8 \alpha_{1} \\
a_{1}^{2}\left(\mathbf{x}(t), \alpha_{2}\right)=8 \alpha_{2} \\
a_{1}^{3}\left(\mathbf{x}(t), \alpha_{3}\right)=32 \alpha_{3}
\end{array}\right.
$$

onde $a_{1}^{i}()$ representa a função de propensidade do $i$-ésimo sistema para a reação de infecção $\mathcal{R}_{1}$.

A probabilidade de um encontro entre um indivíduo suscetível e um infectado depende da quantidade desses elementos na população. Infelizmente, notemos que da maneira como a função de propensidade está formulada, a informação acerca do tamanho populacional do sistema fica incorporada no parâmero $\alpha_{j}$, o que é indesejável.

Então, em epidemiologia é comum uma abordagem levemente diferente da adotada em reações químicas.

Já vimos que a ocorrência de uma infecção está diretamente ligada ao encontro de um indivíduo suscetível com um infectado. Conforme sugerido por Hethcote [Het00], tomando um parâmetro $\beta$ que representa o número médio de contatos adequados (suficientes para infecção) de uma pessoa por unidade de tempo, temos que $\beta I / N$ é o número médio de contatos com infectados por unidade de tempo de um indivíduo suscetível. Daí segue que o número de novos casos da doença por unidade de tempo é representado por $\beta S I / N$.

Então, a função de propensidade para a primeira reação $\mathcal{R}_{1}$ pode ser reescrita como:

$$
a_{1}(\mathbf{x}(t), \beta)=\beta \frac{S(t) I(t)}{N}
$$

onde $N$ representa o tamanho populacional do sistema. Reescrevendo as expressões 2.13 , obtemos:

$$
\left\{\begin{array}{l}
a_{1}^{1}(\mathbf{x}(t), \beta)=\beta_{1} \\
a_{1}^{2}(\mathbf{x}(t), \beta)=\frac{2}{3} \beta_{2} \\
a_{1}^{3}(\mathbf{x}(t), \beta)=2 \beta_{3}
\end{array}\right.
$$

Tomando $\beta_{1}=\beta_{2}=\beta_{3}$, lembrando da definição da função de propensidade, notemos que a probabilidade de ocorrer uma infecção no sistema 1 é maior que no sistema 2, o que é razoável, uma vez que a proporção de indivíduos suscetíveis e infectados é maior nesse sistema, tornando o encontro suscetível-infectado mais provável.

Em 2.15 podemos observar ainda que, embora os indivíduos suscetíveis, infectados e recuperados possuam a mesmas proporções nos sistemas 1 e 3, a probabilidade de ocorrer uma infecção no sistema 3 é duas vezes maior que no sistema 1. Este fato está em conformidade com a teoria, uma vez que, o número de suscetíveis, infectados e recuperados do meio 3 é exatamente o dobro do encontrado no sistema 1. Logo, uma infecção no sistema 1 é proporcionalmente equivalente à duas infecções no sistema 3 .

Analisando agora a função de propensidade para a reação de recuperação $\mathcal{R}_{2}$, podemos notar que, como ela é uma reação de primeira ordem e independe de colisões, independe do tamanho da população. Mais especificamente, o parâmetro $\gamma$ está intimamente ligado ao tempo de recuperação da doença.

Então, dado um estado $\mathbf{X}(t)=(S(t)=S, I(t)=I, R(t)=R)$ em um instante $t$, tomando um 
intervalo infinitesimal $[t, t+\Delta t]$ e lembrando a expressão 2.3:

$$
\begin{aligned}
& P[S(t+\Delta t)=S-1, I(t+\Delta t)=I+1, R(t+\Delta t)=R \mid S(t)=S, I(t)=I, R(t)=R]=\beta \frac{S I}{N} \Delta t \\
& P[S(t+\Delta t)=S, I(t+\Delta t)=I-1, R(t+\Delta t)=R+1 \mid S(t)=S, I(t)=I, R(t)=R]=\gamma I \Delta t \quad(2.16) \\
& P[S(t+\Delta t)=S, I(t+\Delta t)=I, R(t+\Delta t)=R(t) \mid S(t)=S, I(t)=I, R(t)=R]=1-\left[\beta \frac{S}{N}+\gamma\right] I \Delta t
\end{aligned}
$$

Agora que construímos a função de propensidade, o que nos separa de executar o algoritmo de Gillespie é o fato de desconhecermos os parâmetros $\beta$ e $\gamma$. As formas de estimar esses parâmetros é o foco do capítulo a seguir. 


\section{Capítulo 3}

\section{Estimação de parâmetros}

Nesse capítulo, discutiremos algumas metodologias para obter informações que sejam revertidas em estimativas para os parâmetros do modelo a partir de dados obtidos da doença. O primeiro passo nesse sentido é compreender profundamente a natureza dos dados disponíveis.

\subsection{Os dados}

Nossa base de dados foi elaborada através com os dados disponibilizados no site do Ministério da Saúde [SIN16] com o número de notificações de novos casos de dengue por semana em cada cidade, o que nos fornece o número de infectados sintomáticos $I_{s}$ na semana.

Amparados nos dados obtidos no sistema SINAN do Ministério da Saúde [SIN16] para o estado do Rio de Janeiro elaboramos a base de dados de notificações semanais de novos casos de dengue por cidade. Esses dados semanais compreendem 82 municípios do estado do Rio de Janeiro, no período de 200001 a 201401, onde a semana é referenciada no formato $A A A A S S$, onde os primeiros quatro dígitos $A A A A$ correspondem ao ano e os dois últimos $S S$ representam a semana.

Infelizmente a proporção de notificações na base com discriminação do sorotipo corresponde a menos de $5 \%$ de todos os registros de modo que não consideramos razoável elaborar um sistema de equações detalhando a dinâmica de cada sorotipo baseado nesses dados. Além disso, observando a característica sazonal da doença, que costuma se manifestar principalmente no verão, optamos então o modelo é aplicado em cada período de um ano epidemiológico (conforme a hipótese 2.3.1).

A figura 3.1 apresenta o exemplo de um gráfico com o número de notificações de casos de dengue de uma cidade registradas ao longo de um ano epidemiológico. Por ser um período de baixa incidência de dengue, foi tomado a quadragésima semana do ano como referência para a quebra de ano. Dessa forma, tomando, por exemplo o ano epidemiológico de 2012-2013, podemos notar na figura 3.1 que ele compreende da semana 40 do ano 2012 à semana 39 do ano 2013. 


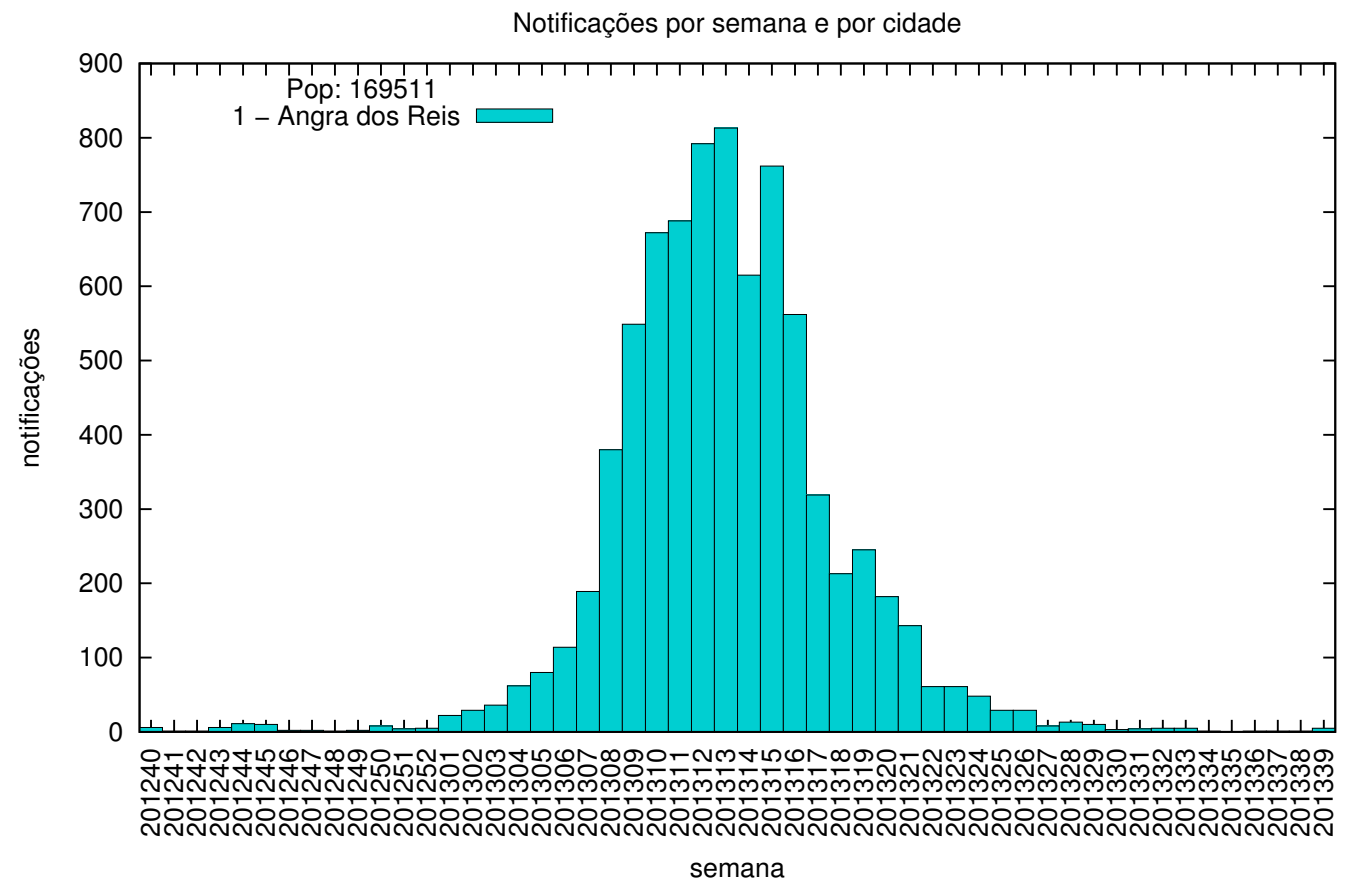

Figura 3.1: Notificações de casos de dengue na cidade de Angra dos Reis no período 2012-2013

\subsection{Inferência através de dados completos}

Antes da iniciação às técnicas de estimação, vamos discutir brevemente o conceito de verossimilhança. Em uma rápida consulta ao dicionário Michaelis [MIC09] observamos que verossimilhança é um substantivo feminino que indica a qualidade do que parece verdadeiro, do que não contraria a verdade.

Em estatística, definimos a função de verossimilhança $L$ de um conjunto de parâmetros $\theta$, dada alguma observação $x$ como a probabilidade de ocorrência da observação $x$ dados os valores dos parâmetros $\theta$.

Em 1912, o biólogo geneticista e estatístico inglês sir Ronald Aylmer Fisher empregou em sua obra On an absolute criterion for fitting frequency curves [Fis97] a função de verossimilhança, onde sugere que os valores de $\theta$ que maximizam a probabilidade de $x$ seriam boas estimativas para o parâmetro $\theta$. A ideia foi retomada em seu artigo de 1922 [Fis22], onde foi proposto o método de máxima verossimilhança para encontrar estimativas pontuais de parâmetros, o estimador de máxima verossimilhança [Edw74, Ald97].

Em nosso problema, desejamos, através dos dados disponíveis, estimar os parâmetros $\boldsymbol{\theta}=$ $\left(\theta_{1}, \theta_{2}\right)=(\beta, \gamma)$.

Primeiramente, vamos considerar um caso idealizado em que se conhece a trajetória completa de todos os componentes do vetor de estados do sistema em um intervalo finito [0,T], ou seja, conhecemos o valor de $\mathbf{X}(t)$, para todo $t$ em $[0, T]$. Notemos que dados com esse formato também podem ser obtidos através da realização de uma simulação do algoritmo de Gillespie no intervalo $[0, T]$ [Wil11].

Conforme descrito na seção 2.4.3, nesse sistema são admitidos dois tipos de reação:

- Tipo 1: um indivíduo suscetível infecta-se após contato com um infectado;

- Tipo 2: um indivíduo infectado recupera-se da enfermidade.

Então, dada uma trajetória, representemos por $r_{1}$ e $r_{2}$ o número de reações observadas do tipo 1 e 2 , respectivamente, no intervalo $[0, T]$. Vamos ainda denotar por $n=r_{1}+r_{2}$, o número total de reações ocorridas nesse intervalo. 
Seja $t_{i}$ o instante da $i$-ésima ocorrência de uma reação no intervalo $[0, T]$. Obtemos então uma sequência $\left(t_{1}, t_{2}, \ldots, t_{n}\right)$ com os instantes de cada reação ocorrida no sistema. Por conveniência, vamos ainda definir: $t_{0}=0$ e $t_{n+1}=T$.

A função de verossimilhança pode ser deduzida rigorosamente através da metodologia de análise de sobrevivência [HJ02]. Podemos observar os tempos de espera como tempo de falha, em que dois eventos de falha são possíveis: uma infecção ou uma recuperação [HJ02]. Vamos, no entanto, adotar uma abordagem simplificada, conforme detalhado em Wilkinson [Wil11], utilizando os termos da probabilidade conjunta de um evento e seu respectivo tempo de reação.

Dada então uma amostra $\mathbf{x}(t)=(S(t), I(t), R(t))$ para $\boldsymbol{X}(t)$ conhecida para todo $t \in[0, T]$, notamos que essa trajetória fica completamente definida pelo estado do sistema no início do intervalo, $\boldsymbol{X}(0)=\mathbf{x}_{0}$, e todos os pares $\left(t_{i}, \xi_{i}\right) \in[0, T] \times\{1,2\}$ com $i=1, \ldots, n$, onde o primeiro elemento da dupla representa o instante de tempo e o segundo, o tipo da $i$-ésima reação no intervalo. É claro que para uma amostra $\mathbf{x}(t)$ ser viável então $S(t), I(t), R(t)$ devem ser inteiros não negativos e devem satisfazer $N=S(t)+I(t)+R(t)$, para todo $t \in[0, T]$, onde $N$ é o número total de indivíduos.

Então, a verossimilhança para um evento $\xi_{i}$ com seu respectivo tempo de espera é dado por:

$$
\begin{aligned}
L_{\mathbf{x}_{i}}(\boldsymbol{\theta}) & =a_{0}\left(\mathbf{x}\left(t_{i-1}\right), \boldsymbol{\theta}\right) \exp \left\{-a_{0}\left(\mathbf{x}\left(t_{i-1}\right), \boldsymbol{\theta}\right)\left[t_{i}-t_{i-1}\right]\right\} \times \frac{a_{\xi_{i}}\left(\mathbf{x}\left(t_{i-1}\right), \theta_{\xi_{i}}\right)}{a_{0}\left(\mathbf{x}\left(t_{i-1}\right), \boldsymbol{\theta}\right)} \\
& =\exp \left\{-a_{0}\left(\mathbf{x}\left(t_{i-1}\right), \boldsymbol{\theta}\right)\left[t_{i}-t_{i-1}\right]\right\} \times a_{\xi_{i}}\left(\mathbf{x}\left(t_{i-1}\right), \theta_{\xi_{i}}\right)
\end{aligned}
$$

Além disso, a probabilidade de que nenhum evento ocorra após a $n$-ésima reação (a última do intervalo $[0, T])$ é dada por:

$$
\exp \left\{-a_{0}\left(\mathbf{x}\left(t_{n}\right), \boldsymbol{\theta}\right)\left[T-t_{n}\right]\right\}
$$

Utilizando as expressões em 3.1 e 3.2 e admitindo a independência entre os tempos de falha, a função de verossimilhança completa é dada observando todas as infecções e recuperações que se deram no intervalo $[0, T]$ [HJ02, Wil11]:

$$
\begin{aligned}
L(\boldsymbol{\theta}, \mathbf{x}) & =\pi(\mathbf{x} \mid \boldsymbol{\theta}) \\
& =\prod_{i=1}^{n} L_{\mathbf{x}_{i}}(\boldsymbol{\theta}) \times \exp \left\{-a_{0}\left(\mathbf{x}\left(t_{n}\right), \boldsymbol{\theta}\right)\left[T-t_{n}\right]\right\} \\
& =\left(\prod_{i=1}^{n} a_{\xi_{i}}\left(\mathbf{x}\left(t_{i-1}\right), \theta_{\xi_{i}}\right)\right)\left(\prod_{i=1}^{n+1} \exp \left\{-a_{0}\left(\mathbf{x}\left(t_{i-1}\right), \boldsymbol{\theta}\right)\left[t_{i}-t_{i-1}\right]\right\}\right) \\
& =\left(\prod_{i=1}^{n} a_{\xi_{i}}\left(\mathbf{x}\left(t_{i-1}\right), \theta_{\xi_{i}}\right)\right) \exp \left(-\sum_{i=0}^{n} a_{0}\left(\mathbf{x}\left(t_{i-1}\right), \boldsymbol{\theta}\right)\left[t_{i}-t_{i-1}\right]\right)
\end{aligned}
$$

Notemos agora que as funções de propensidade para este problema, definidas em 2.11 são separáveis (ver definição2.4.1). Podemos então escrever a expressão 3.3 na forma: 


$$
\begin{aligned}
L(\boldsymbol{\theta}, \mathbf{x}) & =\left(\prod_{i=1}^{n} \theta_{\xi_{i}} g_{\xi_{i}}\left(\mathbf{x}\left(t_{i-1}\right)\right)\right) \exp \left(-\int_{0}^{T} \sum_{j=1}^{2} \theta_{j} g_{j}(\mathbf{x}(t)) d t\right) \\
& =\prod_{i=1}^{n} g_{\xi_{i}}\left(\mathbf{x}\left(t_{i-1}\right)\right) \prod_{j=1}^{2} \theta_{j}^{r_{j}} \exp \left(-\theta_{j} \int_{0}^{T} g_{j}(\mathbf{x}(t)) d t\right) \\
& =\beta^{r_{1}} \gamma^{r_{2}}\left[\prod_{i=1}^{r_{1}} \frac{S\left(t_{i-1}\right) I\left(t_{i-1}\right)}{N}\right]\left[\prod_{i=1}^{r_{2}} I\left(t_{i-1}\right)\right] \exp \left(-\int_{0}^{T} \beta \frac{S(t) I(t)}{N}-\gamma I(t) d t\right)
\end{aligned}
$$

Diferenciando a expressão obtida em relação à $\beta$ e $\gamma$ e igualando a zero, obtemos os estimadores de máxima verossimilhança:

$$
\hat{\beta}=\frac{r_{1}}{\int_{0}^{T} \frac{S(t) I(t)}{N} d t}, \quad \hat{\gamma}=\frac{r_{2}}{\int_{0}^{T} I(t) d t}
$$

Denotando as seguintes quantidades:

$$
g_{\text {prod }}=\prod_{i=1}^{n} g_{\xi_{i}}\left(\mathbf{x}\left(t_{i-1}\right)\right), \quad G_{j}=\int_{0}^{T} g_{j}(\mathbf{x}(t)) d t
$$

podemos reescrever a equação 3.4 :

$$
L(\boldsymbol{\theta}, \mathbf{x})=\pi(\mathbf{x} \mid \boldsymbol{\theta})=g_{\text {prod }} \prod_{j=1}^{2} \theta_{j}^{r_{j}} e^{-\theta_{j} G_{j}}
$$

Como $g_{j}(\mathbf{x}(t))$ é não negativa para $j=1,2$, o que implica em $G_{i}$ também não negativa, então notemos que, conforme observado por Gupta [Gup13], a expressão encontrada para $L(\boldsymbol{\theta}, \mathbf{x})$ se assemelha (exceto por constantes) à função densidade de probabilidade da distribuição gama. Esse fato justifica o frequente emprego de uma distribuição gama à priori.

Definimos então as distribuições a priori:

$$
\begin{aligned}
\theta_{j} & \sim \operatorname{Gama}\left(a_{j}, b_{j}\right), \quad j=1,2 \\
\pi\left(\theta_{j}\right) & =\frac{b_{j}^{a_{j}}}{\Gamma\left(a_{j}\right)} \theta_{j}^{a_{j}-1} e^{-b_{j} \theta_{j}}, \quad j=1,2 \\
\pi(\boldsymbol{\theta}) & =\prod_{j=1}^{2} \pi\left(\theta_{j}\right)=\pi\left(\theta_{1}\right) \pi\left(\theta_{2}\right)
\end{aligned}
$$

Dizemos então que as expressões em 3.6 definem a priori conjugada à verossimilhança [Gup13]. Vamos agora usar a fórmula de Bayes para encontrar a distribuição a posteriori: 


$$
\begin{aligned}
\pi(\boldsymbol{\theta} \mid \mathbf{x}) & =\frac{\pi(\mathbf{x} \mid \boldsymbol{\theta}) \pi(\boldsymbol{\theta})}{\pi(\mathbf{x})} \propto \pi(\mathbf{x} \mid \boldsymbol{\theta}) \pi(\boldsymbol{\theta}) \\
& =g_{\text {prod }} \prod_{i=1}^{2} \theta_{i}^{r_{i}} e^{-\theta_{i} G_{i}} \frac{b_{i}^{a_{i}}}{\Gamma\left(a_{i}\right)} \theta_{i}^{a_{i}-1} e^{-b_{i} \theta_{i}} \\
& \propto \prod_{i=1}^{2} \theta_{i}^{r_{i}+a_{i}-1} e^{-\theta_{i}\left(b_{i}+G_{i}\right)}
\end{aligned}
$$

Como a distribuição a posteriori $\pi(\boldsymbol{\theta} \mid \mathbf{x})$ deve integrar 1 em seu domínio, obtemos:

$$
\begin{aligned}
& \pi(\boldsymbol{\theta} \mid \mathbf{x})=\prod_{i=1}^{2} \frac{\left(b_{i}+G_{i}\right)^{a_{i}+r_{i}}}{\Gamma\left(a_{i}+r_{i}\right)} \theta_{i}^{a_{i}+r_{i}-1} e^{-\theta_{i}\left(b i+G_{i}\right)} \\
& \pi(\boldsymbol{\theta} \mid \mathbf{x})=\prod_{i=1}^{2} \pi\left(\theta_{i} \mid \mathbf{x}\right) \\
& \pi\left(\theta_{i} \mid \mathbf{x}\right)=\operatorname{Gama}\left(a_{i}+r_{i}, b_{i}+G_{i}\right)=\operatorname{Gama}\left(a_{i}+r_{i}, b_{i}+\int_{0}^{T} g_{i}(\mathbf{x}(t)) d t\right), \quad i=1,2
\end{aligned}
$$

onde $\pi\left(\theta_{i} \mid \mathbf{x}\right)$ é chamado de distribuição a posteriori marginal.

Portanto, se a trajetória completa do vetor de estados ao longo de um intervalo de tempo $[0, T]$ está disponível, os parâmetros podem ser estimados usando o estimador de máxima verossimilhança ou através da abordagem Bayesiana.

\subsection{Construção da base de dados discreta}

Como já descrito, o número de notificações registradas é acumulada e armazenada de forma semanal, ou seja, temos informação em apenas um número finito e igualmente espaçados de pontos no tempo. Faz-se portanto necessário tentar uma abordagem diferente da apresentada previamente.

Estamos interessados em estudar a dinâmica da doença no sistema no início do surto. Para isso, vamos definir o período de ajuste, que determina o número de semanas observadas no modelo.

A primeira dificuldade reside no fato de que é difícil definir o início da epidemia uma vez que notificações de casos de dengue são efetuadas durante praticamente o ano todo, como pode ser visto nos exemplos retratados na figura 3.2.

Notemos que durante um período a infecção se apresenta sob um regime letárgico em que se verifica uma pequena oscilação do número de notificações, o que atesta a presença do agente infeccioso, entretanto sem o potencial de se transformar em uma epidemia. Em algum momento esse regime é rompido e o número de infectados começa a crescer. A identificação desse instante é fundamental para o estudo da dinâmica da epidemia.

Através de observações em gráficos de notificações, como os obtidos na figura 3.1, determinamos o início do surto epidêmico, isto é, o instante em que o número de infectados torna-se relevante para que haja um crescimento da doença. Identificamos esse instante como $t=t_{0}$.

Definiremos então o período de ajuste de tamanho $T+1$ formado pela semana $t=t_{0}$ e as $T$ semanas subsequentes. Renomeando o instante inicial $t_{0}$ como a semana 0 podemos, sem perda de generalidade, denotar o intervalo $\left[t_{0}, t_{0}+T\right]$ pelo intervalo de mesmo comprimento $[0, T]$.

Infelizmente o número exato de indivíduos suscetíveis, infectados e recuperados (o estado do sistema) em cada semana do período de ajuste não está disponível de forma direta. Ela pode ser obtida indiretamente através dos dados de notificações semanais, encontrada no sistema no Ministério da Saúde [SIN16]. 


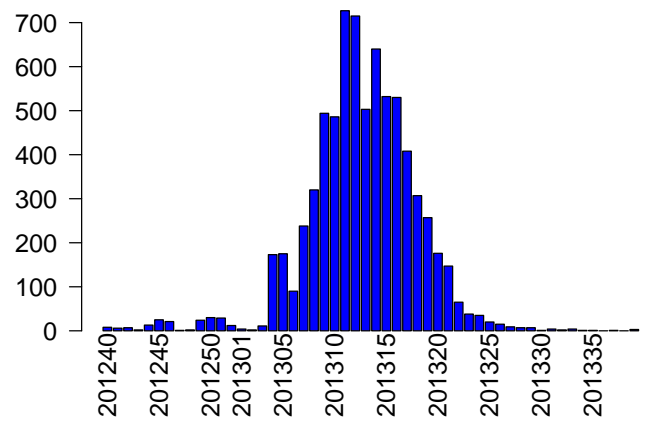

(a) Cabo Frio

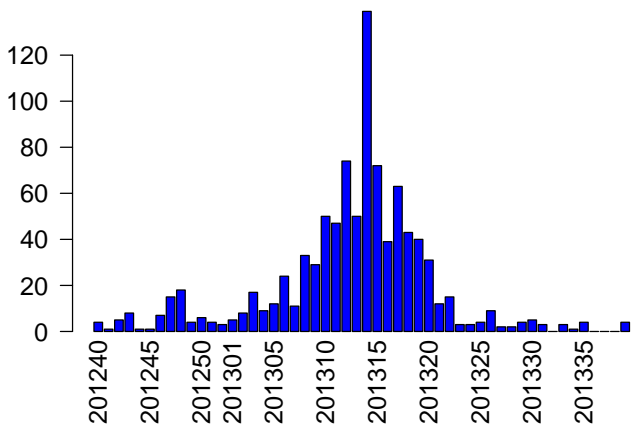

(c) Nova Iguaçu

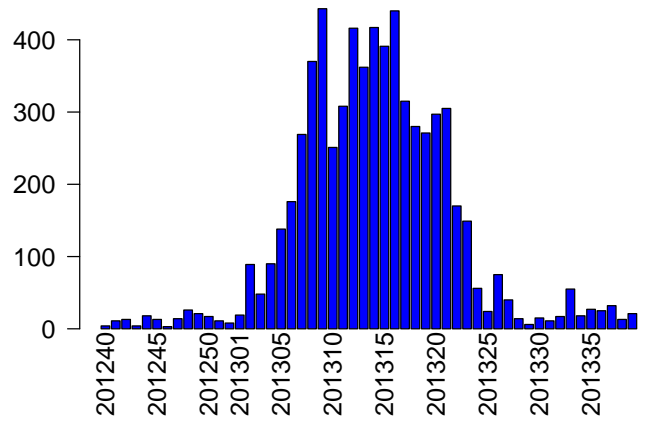

(b) Duque de Caxias

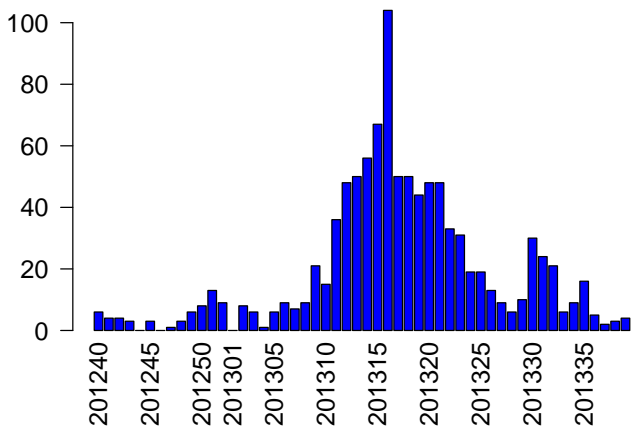

(d) São João de Meriti

Figura 3.2: Notificações em cidades do estado do Rio de Janeiro no período 2012-2013

O número de notificações de uma cidade ao longo do tempo é armazenada em um vetor Not. Denotaremos o número registrado de casos da doença na $j$-ésima semana $N o t[j]$.

Dada uma semana $j$, o número de notificações $N o t[j]$ fornece uma estimativa para o número de infecções sintomáticas da doença ocorridas entre as semanas $[j-1, j]$. Utilizando a relação definida na hipótese 2.3.2, obtemos uma projeção do número de novos infectados para cada semana:

$$
I_{\text {proj }}[j]=(1+\mu) \operatorname{Not}[j]
$$

onde $I_{\text {proj }}[j]$ representa o número de novas infecções projetadas ocorridas entre as semanas $[j-1, j]$ e $\mu>0$ é um parâmetro fixado.

Um grande problema observado nos dados de notificação de dengue é a grande oscilação, muitas vezes inesperada no número de registros. Ela pode ser ocasionada por diversos fatores, sejam eles associados à doença, ou não, no caso de displicência no registro das notificações, por exemplo. Tais oscilações prejudicam a acurácia de estimadores, sobretudo os baseados em funções de verossimilhança. Com a finalidade de atenuar tais ruídos sem comprometer a tendência assinalada nos dados, foi utilizada a suavização por médias móveis simples (MMS) [MT06].

Utilizando um período de três semanas para a média móvel simples, obtemos uma fórmula alternativa a 3.10 :

$$
I_{\text {proj } j}[j]=\left\{\begin{array}{l}
\operatorname{round}\left((1+\mu) \frac{N o t[j-2]+N o t[j-1]+N o t[j]}{3}\right), \quad \text { se } j \geq 3 \\
\operatorname{round}((1+\mu) N o t[j]), \quad \text { caso contrário }
\end{array}\right.
$$


onde $\operatorname{round}()$ é função de arredondamento, lembrando que o número de infectados é um inteiro positivo.

A figura 3.3 apresenta o efeito obtido pela suavização através da média móvel simples de três semanas.

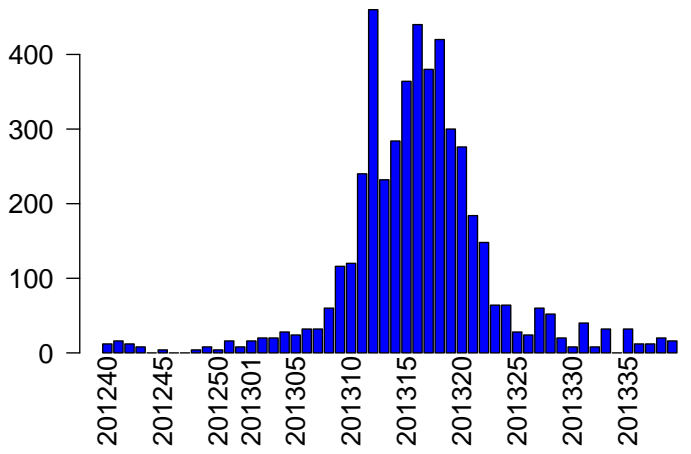

(a) Infectados projetados em Belford Roxo sem suavização

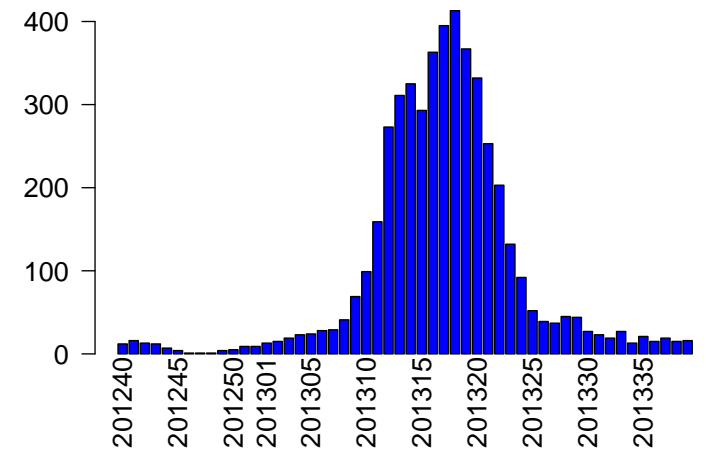

(b) Infectados projetados em Belford Roxo suavizados

Figura 3.3: Efeito da suavização por médias móveis simples no número de infectados projetados

Baseados na projeção semanal do número de novos infectados $I_{\text {proj }}$, podemos construir a sequência (vetorial) $\mathbf{x}_{0}, \mathbf{x}_{1}, \ldots, \mathbf{x}_{T}$ dos estados do sistema ao longo do período de ajuste através da regra descrita a seguir.

Dada a semana inicial $t_{0}$ escolhida com base em observações de $I_{p r o j}$, então:

- Para a primeira semana do período de ajuste (semana 0):

$$
\begin{aligned}
S_{0} & =N-I_{\text {proj }}\left[t_{0}\right] ; \\
I_{0} & =I_{\text {proj }}\left[t_{0}\right] ; \\
R_{0} & =0
\end{aligned}
$$

onde $N$ representa o tamanho populacional da região estudada.

- Para as demais semanas, $j=1, \ldots, T$ :

$$
\begin{aligned}
S_{j} & =S_{j-1}-I_{\text {proj }}\left[t_{0}+j\right] \\
I_{j} & =I_{\text {proj }}\left[t_{0}+j\right] \\
R_{j} & =R_{j-1}+I_{j-1}
\end{aligned}
$$

Notemos que nessa abordagem, consideramos não haver infecção anterior à semana $0, \operatorname{logo}$ a população é formada apenas por indivíduos suscetíveis, conforme a hipótese 2.3.1. É nesse momento (semana 0) em que os primeiros $I_{\text {proj }}\left[t_{0}\right]$ indivíduos se infectam e a dinâmica da infecção se inicia no modelo, conforme observado nas equações 3.12.

Infelizmente inexiste um sistema de notificação para a recuperação dos indivíduos infectados, e como já discutido no seção 2.2, estima-se que um indivíduo leve de 4 até 10 ou 12 dias para a recuperação. Nesse trabalho admitiremos que o indivíduo infectado leve, no máximo, uma semana (7 dias) para se reestabelecer. Desse modo, espera-se que um indivíduo infectado na $j$-ésima semana esteja recuperado na $(j+1)$-ésima semana. Notemos que esse fato foi considerado na construção da sequência $\mathbf{x}_{0}, \ldots, \mathbf{x}_{T}$ através das expressões em 3.13 . 


\subsection{Inferência através de observações em tempo discreto}

Construída a sequência $\left\{\mathbf{x}_{j}, j=0, \ldots, T\right\}$ que descreve a evolução semana a semana do sistema estudado, conforme documentado na seção 3.3, vamos apresentar a teoria de inferência através de observações em tempo discreto.

Representaremos a variável aleatória que denota o estado do sistema ao longo do intervalo $[0, T]$ por $\mathbf{X}=\{\mathbf{X}(t), t \in[0, T]\}$, onde $\mathbf{X}(t)=(\mathbf{S}(t), \mathbf{I}(t), \mathbf{R}(t))$. Já uma amostra (trajetória) de $\mathbf{X}$ será denotada por $\mathbf{x}=\{\mathbf{x}(t), t \in[0, T]\}$ com $\mathbf{x}(t)=(S(t), I(t), R(t))$.

Uma trajetória $\mathbf{x}$ é dita viável quando as seguintes condições são satisfeitas:

- $\mathbf{x}(t)=\mathbf{X}(t)=\mathbf{x}_{t}, t=0, \ldots, T$

- $S(t) \geq 0, I(t) \geq 0, R(t) \geq 0, t \in[0, T]$

- $S(t)+I(t)+R(t)=N$, onde $N$ representa o tamanho populacional da região estudada.

Notemos que o problema de gerar uma trajetória dados os parâmetros $\boldsymbol{\theta}=(\beta, \gamma)$ e um ponto inicial $\mathbf{x}_{t}$ pode ser resolvida diretamente através da simulação do algoritmo de Gillespie (ver algoritmo 1). Entretanto, quando a condição de ponto final $\mathbf{x}_{t+1}$ é inserida, o problema se torna muito mais complexo e uma nova estratégia deve ser adotada.

Vamos construir um algoritmo MCMC para gerar subtrajetórias condicionadas aos estados nos pontos inicial e final observados e aos parâmetros $(\beta$ e $\gamma)$ para cada intervalo $[t, t+1], t \in 0, \ldots, T-1$. A união de todas as subtrajetórias dá origem à uma trajetória completa. A iteração é completada pela amostragem dos parâmetros condicionada à trajetória completa.

Gostaríamos de gerar uma amostra diretamente da distribuição $(\mathbf{x} \mid \boldsymbol{\theta}, \mathbf{x}(0), \ldots, \mathbf{x}(T))$. Entretanto, diante da dificuldade de tal tarefa, iremos proceder conforme sugerido por Wilkinson [Wil11], selecionando as amostras $\mathbf{x}$ através do procedimento de atualização de Metropolis-Hastings com distribuição alvo $\pi(\mathbf{x} \mid \boldsymbol{\theta}, \mathbf{x}(0), \ldots, \mathbf{x}(T))$.

Notemos que conhecendo-se os estados $\mathbf{x}_{j}$ e $\mathbf{x}_{j+1}$ correspondentes, respectivamente, ao ponto inicial e final de um subintervalo do período de ajuste, ficam completamente determinadas o número de reações do tipo 1 e 2 ocorridas no período. Então, para determinar uma trajetória nesse subintervalo basta determinar a sequência e o instante no tempo em que se dão essas reações. Lembramos ainda que as relações da ocorrência das reações são determinadas pelas funções de propensidade, que dependem do estado do sistema e, portanto são conhecidas no ponto inicial e final de cada subintervalo. A ideia é, utilizando essa informação, aproximar o processo reativo para cada tipo de reação através de um processo não homogêneo de Poisson em que a taxa, varia linearmente entre as propensidades inicial e final em um procedimento semelhante ao block updating method apresentado no trabalho de Boys, Wilkinson, Kirkwood [BWK08].

Apresentaremos agora alguns resultados úteis da teoria de processos estocásticos que complementam a teoria básica apresentada na seção A.1.

Proposição 3.4.1. Considere um processo de Poisson homogêneo com taxa $\lambda$ em um intervalo finito $[0, T]$. Seja $R \sim$ Poisson $(\lambda T)$ o número de eventos. Então, condicionado a $R$, os tempos de ocorrência dos eventos são variáveis aleatórias com distribuição Unif $(0, T)$.

A demonstração desse fato será omitida, podendo ser consultada no trabalho de Wilkinson [Wil11].

A proposição 3.4.1 estabelece uma maneira de simular um processo homogêneo de Poisson condicionado ao número de eventos. O resultado a seguir, apresentado no trabalho de Boys, Wilkinson e Kirkwood [BWK08], estabelece uma relação entre os tempos dos eventos de um processo homogêneo e um processo não homogêneo com a taxa como função linear no tempo.

Proposição 3.4.2. Sejam $\mathbf{X}$ um processo homogêneo de Poisson em um intervalo $[j, j+1]$ com taxa $\mu=\frac{a_{j}+a_{j+1}}{2}$ e $\mathbf{Y}$ um processo não homogêneo de Poisson com taxa dada por

$$
\lambda(t)=(j+1-t) a_{j}+(t-j) a_{j+1}, \quad a_{j} \neq a_{j+1}, \quad a_{j}, a_{j+1}>0 .
$$


Então, uma realização do processo $\mathbf{Y}$ pode ser obtida a partir de uma realização do processo $\mathbf{X}$, através da transformação de tempo:

$$
t=j+\frac{\sqrt{a_{j}^{2}+\left[a_{j+1}^{2}-a_{j}^{2}\right](s-j)}-a_{j}}{a_{j+1}-a_{j}},
$$

para todos os tempos de eventos do processo $\mathbf{X}$ (aqui, os tempos do processo homogêneo $\mathbf{X}$ são denotadados pela variável s e os tempos do processo não homogêneo $\mathbf{Y}$ é dada pela variável $t$ ).

Demonstração. Definimos a função acumulada da taxa de $\mathbf{X}$ por $M(s)=\frac{\left(a_{j}+a_{j+1}\right)(s-j)}{2}$. Da mesma forma para $\mathbf{Y}$ temos:

$$
\Lambda(t)=\int_{j}^{t}(1+j-w) a_{j}+(w-j) a_{j+1} d w=a_{j}(t-j)+\left(a_{j+1}-a_{j}\right) \frac{(t-j)^{2}}{2}
$$

Notemos que:

$$
\begin{aligned}
M(j) & =\Lambda(j)=0 \\
M(j+1) & =\Lambda(j+1)=\frac{a_{j}+a_{j+1}}{2}
\end{aligned}
$$

Fazendo agora $M(s)=\Lambda(t)$, temos:

$$
\begin{aligned}
& a_{j}(t-j)+\left(a_{j+1}-a_{j}\right) \frac{(t-j)^{2}}{2}-\frac{\left(a_{j}+a_{j+1}\right)(s-j)}{2}=0 \Leftrightarrow \\
& t^{2} \frac{\left(a_{j+1}-a_{j}\right)}{2}+t\left[a_{j}-j\left(a_{j+1}-a_{j}\right)\right]+\frac{j^{2}\left(a_{j+1}-a_{j}\right)}{2}-j a_{j}-\frac{\left(a_{j}+a_{j+1}\right)}{2}(s-j)=0
\end{aligned}
$$

Resolvendo a equação e tomando a raiz positiva:

$$
t=j+\frac{\sqrt{a_{j}^{2}+\left(a_{j+1}^{2}-a_{j}^{2}\right)(s-j)}-a_{j}}{a_{j+1}-a_{j}}
$$

Portanto, todo processo de Poisson não homogêneo com taxa linearizada em um subintervalo $[j, j+1] \subseteq[0, T]$ pode ser aproximado por um processo de Poisson homogêneo, conforme descrito na proposição 3.4.2, através da transformação do tempo descrito na equação 3.14. Apresentamos abaixo um pseudocódigo (ver algoritmo 3) exemplificando a metodologia.

Utilizamos a informação contida nos vetores de instantes de infecções e recuperações ocorridas em $[j, j+1]$ para construir a evolução do estado do sistema ao longo do intervalo. Como já mencionado no capítulo 2, a evolução do sistema no decorrer do tempo pode ser descrito por uma sequência de pares $\left(t_{n}, \xi_{n}\right)$ onde o primeiro elemento representa o instante e o segundo o tipo de reação ocorrido. Vamos denotar por $\mathbf{x}\left(t_{n}, \xi_{n}\right)$ a evolução do estado do sistema ao longo do tempo, dada uma sequência de pares $\left\{\left(t_{n}, \xi_{n}\right)\right\}$. O pseudocódigo (algoritmo 3 ) descreve o procedimento.

Notemos que durante a execução do algoritmo 3, devemos verificar a condição: $I>0$ pois, em caso contrário, a infecção se encerra. Se, eventualmente, em algum momento for observado $I=0$, a trajetória deve ser descartada e novas sequências de tempo devem ser geradas a partir do algoritmo 2 . 


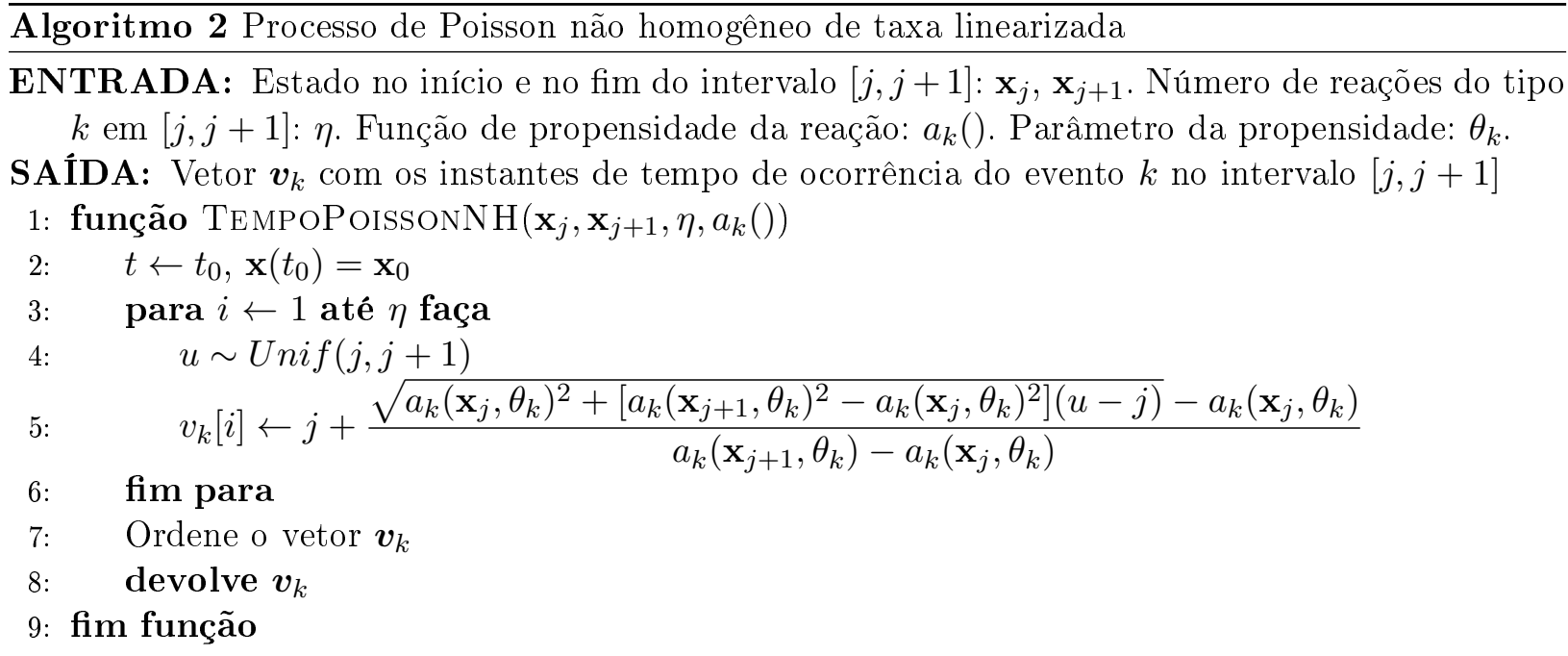

$\overline{\text { Algoritmo } 3 \text { Geração de trajetória através de processo de Poisson não homogêneo de taxa linea- }}$ rizada

ENTRADA: Estado no início do intervalo $[j, j+1]: \mathbf{x}_{j}$. Número de infecções e recuperações ocorridos no período: $\eta_{1}, \eta_{2}$. Vetores representando os instantes de infecção e recuperação gerados pelo algoritmo 2: $\boldsymbol{v}_{1}, \boldsymbol{v}_{2}$

SAÎDA: vetores $\boldsymbol{t}$ e $\boldsymbol{\xi}$ que descrevem o instante de ocorrência e tipo (1 para infecção e 2 para recuperação) do evento

1: função Trajetoria $\left(\mathbf{x}_{j}, \eta_{1}, \eta_{2}, \boldsymbol{v}_{1}, \boldsymbol{v}_{2}\right)$

2: $\quad I \leftarrow I_{j}$

3: $\quad i_{1} \leftarrow 1, i_{2} \leftarrow 1, k \leftarrow 1$

4: $\quad$ enquanto $i_{1} \leq \eta_{1}, i_{2} \leq \eta_{2}$ e $I>0$ faça

$$
\begin{aligned}
& I \leftarrow I+1, i_{1} \leftarrow i_{1}+1 \\
& \text { senão }
\end{aligned}
$$$$
t[k] \leftarrow v_{1}\left(i_{1}\right), \xi[k] \leftarrow 1
$$

$$
\begin{aligned}
& t[k] \leftarrow v_{2}\left(i_{2}\right), \xi[k] \leftarrow 2 \\
& I \leftarrow I-1, i_{2} \leftarrow i_{2}+1
\end{aligned}
$$

\section{fim se}

$k \leftarrow k+1$

\section{fim enquanto}

enquanto $i_{1} \leq \eta_{1}$ faça

$$
\begin{aligned}
& t[k] \leftarrow v_{1}\left(i_{1}\right), \xi[k] \leftarrow 1 \\
& i_{1} \leftarrow i_{1}+1, k \leftarrow k+1
\end{aligned}
$$

\section{fim enquanto}

$$
\text { enquanto } i_{2} \leq \eta_{2} \text { e } I>0 \text { faça }
$$

$t[k] \leftarrow v_{2}\left(i_{2}\right), \xi[k] \leftarrow 2$

$I \leftarrow I-1, i_{2} \leftarrow i_{2}+1, k \leftarrow k+1$

\section{fim enquanto}

se $I \leq 0$ então devolve ERRO

\section{fim se}

$$
\text { devolve } \mathbf{x}(\boldsymbol{t}, \boldsymbol{\xi})=(\boldsymbol{t}, \boldsymbol{\xi})
$$

\section{: fim função}

$\triangleright$ Verificação de viabilidade $\triangleright$ Novos vetores de tempo $\boldsymbol{v}_{1}$ e $\boldsymbol{v}_{2}$ devem ser gerados

$\triangleright$ Devolve o par $(\boldsymbol{t}, \boldsymbol{\xi})$ que define uma trajetória $\mathbf{x}(\boldsymbol{t}, \boldsymbol{\xi})$

Lembramos que $n=r_{1}+r_{2}$ define o número de eventos (infecções e recuperações) ocorridos no intervalo $[0, T]$. Antes de prosseguir, vamos definir a sequência não negativa $\left\{n_{j}\right\}$, com $j=$ 
$0,1, \ldots, T$, onde $n_{j}$ representa o número de reações ocorridas até a $j$-ésima semana. Temos então que: $0=n_{0}<n_{1}<\ldots<n_{T}=n$.

Sejam então $\mathbf{x}_{[0,1]}, \mathbf{x}_{[1,2]} \ldots, \mathbf{x}_{[T-1, T]}$ as subtrajetórias construídas através do algoritmo 3 , em que $\mathbf{x}_{[j, j+1]}$ refere-se à subtrajetória no intervalo $[j, j+1]$. Notemos que a união de todas essas trajetórias definem uma trajetória do sistema no intervalo $[0, T]$, que denotamos por $\mathbf{x}$.

Considere agora uma trajetória inicial $\mathbf{x}=\left\{\mathbf{x}_{[0,1]}, \mathbf{x}_{[1,2]} \ldots, \mathbf{x}_{[T-1, T]}\right\}$, e uma trajetória alternativa $\mathbf{y}=\left\{\mathbf{y}_{[0,1]}, \mathbf{y}_{[1,2]}, \ldots, \mathbf{y}_{[T-1, T]}\right\}$. Uma nova trajetória é formada através da metodologia de Metropolis-Hastings para cada subtrajetória. Ou seja, para cada subintervalo $[j, j+1]$, calcula-se a probabilidade de aceitação $A$, segundo o método de Metropolis-Hastings (que será detalhado adiante) e aceita-se a subtrajetória $\mathbf{x}_{[j, j+1]}$ ou $\mathbf{y}_{[j, j+1]}$ segundo uma regra baseada em $A$.

Antes de apresentar o cálculo da probabilidade de aceitação do Método de Metropolis-Hastings, vamos definir a verossimilhança do modelo definido pelo processo de Poisson não homogêneo com taxa linearizada em cada subintervalo.

Proposição 3.4.3. A verossimilhança para uma trajetória $\mathbf{x}$ em um intervalo $[j, j+1] \subseteq[0, T]$ sob aproximação de um processo não homogêneo de Poisson é dado por:

$$
L_{A_{j}}(\boldsymbol{\theta}, \mathbf{x})=\left[\prod_{i=n_{j}+1}^{n_{(j+1)}} \lambda_{\xi_{i}}\left(t_{i}\right)\right] \exp \left(-\frac{1}{2}\left[a_{0}(\mathbf{x}(j), \boldsymbol{\theta})+a_{0}(\mathbf{x}(j+1), \boldsymbol{\theta})\right]\right)
$$

onde:

$$
\lambda_{k}(t)=(j+1-t) a_{k}\left(\mathbf{x}(j), \theta_{k}\right)+(t-j) a_{k}\left(\mathbf{x}(j+1), \theta_{k}\right), \quad t \in[j, j+1]
$$

$\operatorname{com} k=1,2$.

Demonstração. Vamos proceder de forma semelhante à apresentada por Wilkinson [Wil11], primeiramente definindo:

$$
\begin{aligned}
\Lambda_{k}(t) & =\int_{j}^{t} \lambda_{k}(s) d s, \quad k=1,2, \quad t \in[j, j+1] \\
\lambda_{0}(t) & =\lambda_{1}(t)+\lambda_{2}(t), \quad \Lambda_{0}(t)=\Lambda_{1}(t)+\Lambda_{2}(t)
\end{aligned}
$$

Então, para um $i$-ésimo evento tal que: $t_{i} \in[j, j+1]$, temos que a verossimilhança é dado por:

$$
\lambda_{\xi_{i}}\left(t_{i}\right) \exp \left(-\left[\Lambda_{0}\left(t_{i}\right)-\Lambda_{0}\left(t_{i-1}\right)\right]\right)
$$

A probabilidade de não ocorrer nenhuma reação após a última registrada no intervalo $[j, j+1]$ ocorrer é dada por:

$$
\exp \left(-\left[\Lambda_{0}(j+1)-\Lambda_{0}\left(t_{n_{(j+1)}}\right)\right]\right) .
$$

Combinando todos os eventos do período, obtemos: 


$$
\begin{aligned}
L_{A_{j}}(\boldsymbol{\theta}, \mathbf{x}) & =\pi_{A}(\mathbf{x} \mid \boldsymbol{\theta})=\left[\prod_{i=n_{j}+1}^{n_{(j+1)}} \lambda_{\xi_{i}}\left(t_{i}\right) \exp \left(-\left[\Lambda_{0}\left(t_{i}\right)-\Lambda_{0}\left(t_{i-1}\right)\right]\right)\right] \times \exp \left(-\left[\Lambda_{0}(j+1)-\Lambda_{0}\left(t_{n_{(j+1)}}\right)\right]\right) \\
& =\left[\prod_{i=n_{j}+1}^{n_{(j+1)}} \lambda_{\xi_{i}}\left(t_{i}\right)\right] \exp \left(-\sum_{i=n_{j}+1}^{n_{(j+1)}}\left[\Lambda_{0}\left(t_{i}\right)-\Lambda_{0}\left(t_{i-1}\right)\right]\right) \times \exp \left(-\left[\Lambda_{0}(j+1)-\Lambda_{0}\left(t_{n_{(j+1)}}\right)\right]\right) \\
& =\prod_{i=n_{j}+1}^{n_{(j+1)}} \lambda_{\xi_{i}}\left(t_{i}\right) \exp \left(-\Lambda_{0}(j+1)\right) \\
& =\prod_{i=n_{j}+1}^{n_{(j+1)}} \lambda_{\xi_{i}}\left(t_{i}\right) \exp \left(-\left[\Lambda_{1}(j+1)+\Lambda_{2}(j+1)\right]\right) \\
& =\prod_{i=n_{j}+1}^{n_{(j+1)}} \lambda_{\xi_{i}}\left(t_{i}\right) \exp \left(-\left[\frac{a_{1}(\mathbf{x}(j), \beta)+a_{1}(\mathbf{x}(j+1), \beta)}{2}+\frac{a_{2}(\mathbf{x}(j), \gamma)+a_{2}(\mathbf{x}(j+1), \gamma)}{2}\right]\right) \\
& =\prod_{i=n_{j}+1}^{n_{(j+1)}} \lambda_{\xi_{i}}\left(t_{i}\right) \exp \left(-\frac{1}{2}\left[a_{0}(\mathbf{x}(j), \boldsymbol{\theta})+a_{0}(\mathbf{x}(j+1), \boldsymbol{\theta})\right]\right)
\end{aligned}
$$

Considere agora $L_{j}(\boldsymbol{\theta}, \mathbf{x})$ a verossimilhança sobre dados completos (ver as expressões em 3.4) restrito ao intervalo $[j, j+1]$ :

$$
L_{j}(\boldsymbol{\theta}, \mathbf{x})=\left(\prod_{i=n_{j}+1}^{n_{(j+1)}} a_{\xi_{i}}\left(\mathbf{x}\left(t_{i-1}\right), \theta_{\xi_{i}}\right)\right) \exp \left(-\int_{j}^{j+1} a_{0}(\mathbf{x}(t), \boldsymbol{\theta}) d t\right)
$$

Obtemos então a razão de verossimilhanças:

$$
\begin{aligned}
\frac{L_{j}(\boldsymbol{\theta}, \mathbf{x})}{L_{A_{j}}(\boldsymbol{\theta}, \mathbf{x})}= & \left(\prod_{i=n_{j}+1}^{n_{(j+1)}} \frac{a_{\xi_{i}}\left(\mathbf{x}\left(t_{i-1}\right), \theta_{\xi_{i}}\right)}{\lambda_{\xi_{i}}\left(t_{i}\right)}\right) \\
& \times \exp \left(\frac{a_{0}(\mathbf{x}(j), \boldsymbol{\theta})+a_{0}(\mathbf{x}(j+1), \boldsymbol{\theta})}{2}-\int_{j}^{j+1} a_{0}(\mathbf{x}(t), \boldsymbol{\theta}) d t\right)
\end{aligned}
$$

Notemos que essa razão, expressa em 3.17, quantifica o grau de confinidade da aproximação obtida pelo processo de Poisson não homogêneo com taxa linearizada em relação ao processo de salto Markoviano que representa o processo "real".

Então, em cada iteração do método de Metropolis-Hastings temos uma trajetória (inicial) x. É proposta uma trajetória alternativa $\mathbf{y}$, em que cada subtrajetória $\mathbf{y}_{[j, j+1]}$ deve ser aceita como substituto de $\mathbf{x}_{[j, j+1]}$, com probabilidade $\min \left\{1, A_{j}\right\}$, onde:

$$
A_{j}=\frac{L_{j}(\boldsymbol{\theta}, \mathbf{y})}{L_{A_{j}}(\boldsymbol{\theta}, \mathbf{y})} / \frac{L_{j}(\boldsymbol{\theta}, \mathbf{x})}{L_{A_{j}}(\boldsymbol{\theta}, \mathbf{x})}
$$

Após a obtenção da nova trajetória $\mathbf{x}$ através do mecanismo descrito acima, são obtidos os novos valores para os parâmetros $\beta$ e $\gamma$ através da distribuição a posteriori no intervalo completo $[0, T]$ (ver expressão em 3.9). O código em 4 descreve o procedimento adotado.

A figura 3.4 apresenta a trajetória para a cidade de Angra dos Reis, obtida através de um passo 


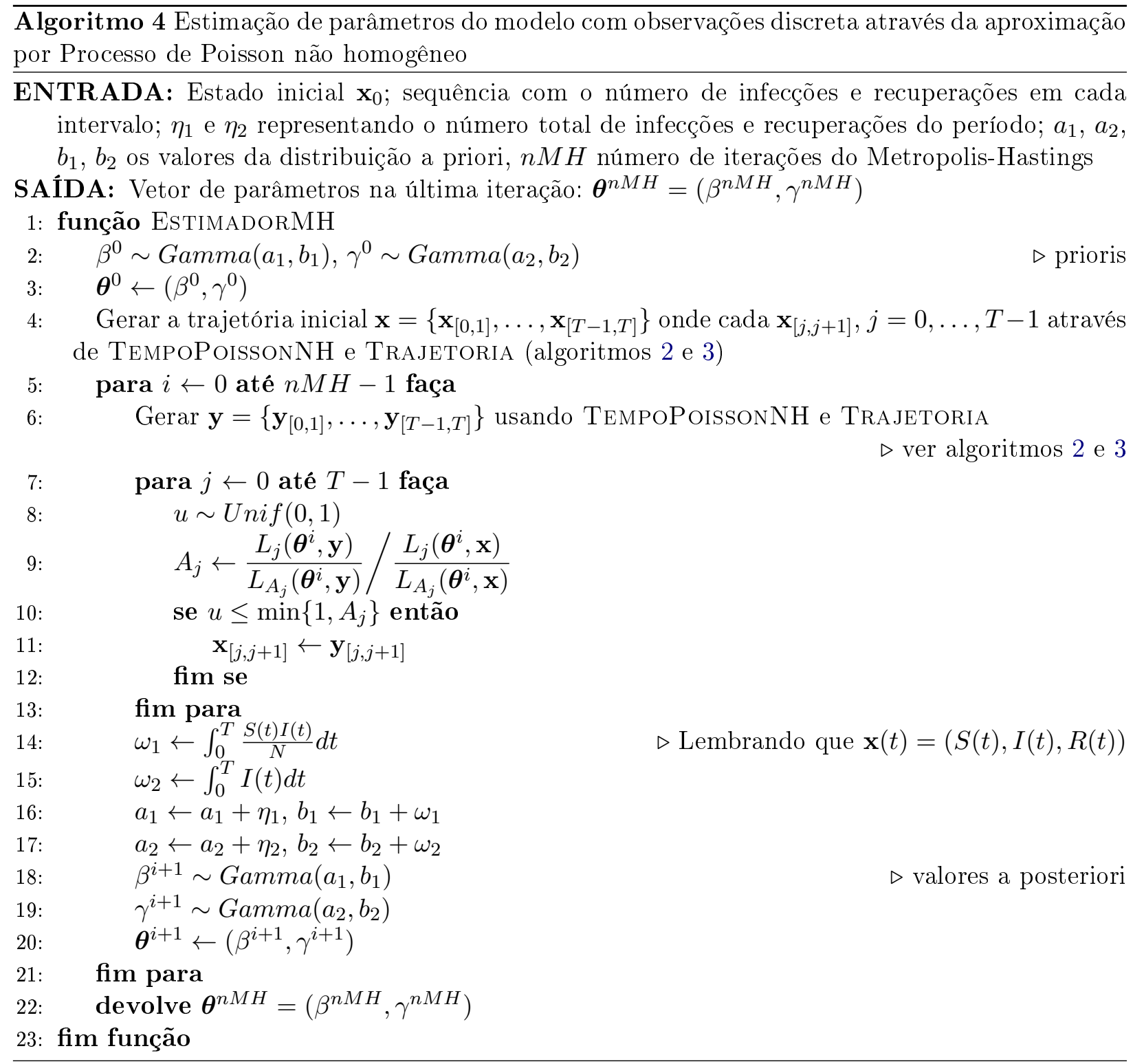

do Método de Metropolis-Hastings, descrito pelo algoritmo 4. O exemplo retrata o surto epidêmico ocorrido no ano 2012-2013. Definimos um período de ajuste de cinco semanas e fixamos 201301 como a semana inicial da infecção, i.e, a semana 201301 é referenciada na figura como a semana 0.

Em 3.4a a trajetória inicial $\mathbf{x}=\left\{\mathbf{x}_{[0,1]}, \ldots, \mathbf{x}_{[4,5]}\right\}$ é representada em azul e em rosa temos a trajetória alternativa $\mathbf{y}=\left\{\mathbf{y}_{[0,1]}, \ldots, \mathbf{y}_{[4,5]}\right\}$. Ambas as trajetórias são geradas através da aproximação por Processos de Poisson não homogêneos (algoritmos 2 e 3). Podemos observar ainda que, por construção, os pontos inicial e final de cada intervalo são coincidentes. Atualizamos então a trajetória resultante através do cálculo da probabilidade de aceitação de Metropolis-Hastings para cada segmento $\mathbf{x}_{[j, j+1]}, j=0, \ldots, 4$.

Na figura $3.4 \mathrm{~b}$ observamos a trajetória resultante $\tilde{\mathbf{x}}=\left\{\tilde{\mathbf{x}}_{[0,1]}, \ldots, \tilde{\mathbf{x}}_{[4,5]}\right\}$ representada em amarelo. Notemos que $\tilde{\mathbf{x}}$ mantém da trajetória inicial apenas o trecho entre os instantes 0 e 1, ou seja, $\tilde{\mathbf{x}}=\left\{\mathbf{x}_{[0,1]}, \mathbf{y}_{[1,2]}, \mathbf{y}_{[2,3]}, \mathbf{y}_{[3,4]}, \mathbf{y}_{[4,5]}\right\}$. Fazemos então $\mathbf{x}=\tilde{\mathbf{x}}$, e um novo passo do Metropolis-Hastings pode ser executado.

Antes de prosseguirmos, vamos ressaltar um fato relevante: em estatística, a qualidade do estimador em geral depende da quantidade de informação disponível. A repetição do mesmo evento, como o lançamento de uma moeda, por exemplo, nos fornece informações acerca da natureza do processo que nos permitem, nesse caso decidir se a moeda é viciada ou não.

Infelizmente no caso de epidemias é impossível replicação do evento estudado, em outras pala- 


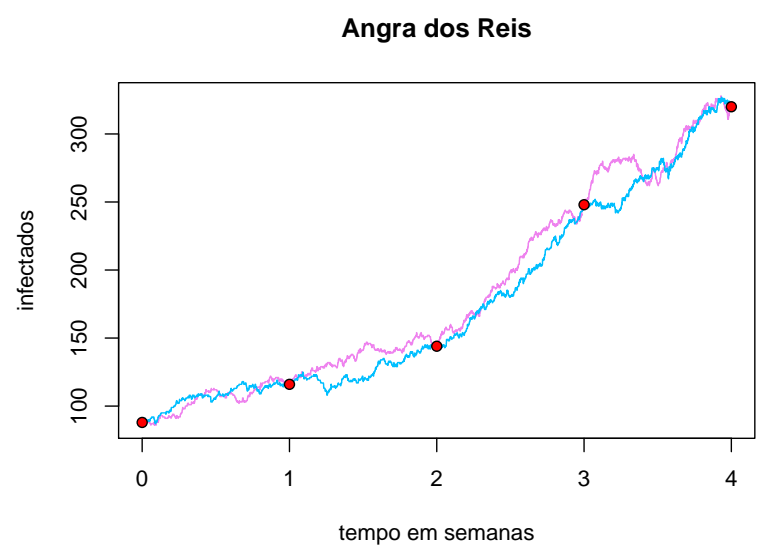

(a) Trajetória inicial $\mathbf{x}$ em azul e trajetória alternativa y em rosa

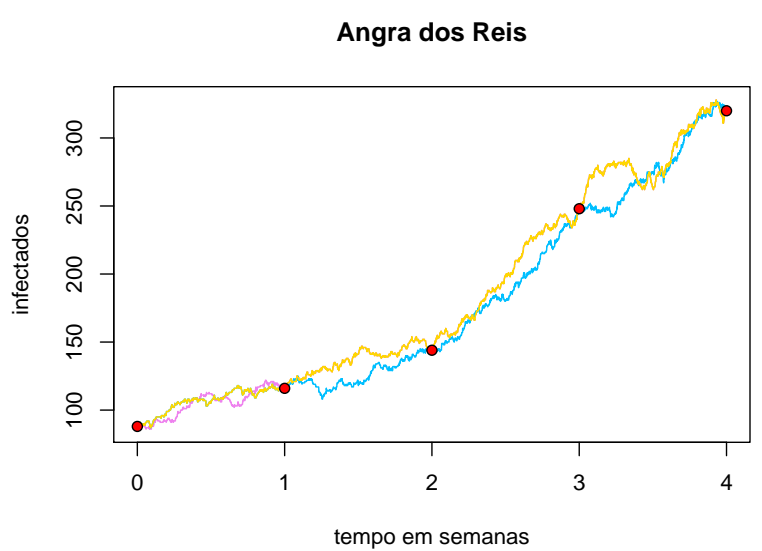

(b) Trajetória inicial $\mathbf{x}$ em azul, trajetória alternativa $\mathbf{y}$ em rosa e a resultante em amarelo

Figura 3.4: Exemplo de construção de trajetória através de um passo do método de Metropolis-Hastings

vras, não podemos reiniciar a infecção e observar os seus desdobramentos.

Por outro lado, segundo a hipótese 2.3.1 considerada na construção do modelo, assumimos que o mesmo agente infeccioso é o responsável pela epidemia deflagrada em todas as cidades. Assim sendo, é razoável supor que, para cidades com perfil demográfico semelhante os parâmetros de infecção e recuperação devem ser próximos. Podemos então realizar a inferência a partir dos dados de um grupo de cidades com perfil demográfico semelhante através de uma simples modificação do algoritmo 4 .

Selecionando então $M$ cidades com características semelhantes e fixando uma semana inicial para cada uma delas, podemos obter os parâmetros do modelo através do algoritmo 5 .

A principal vantagem da abordagem apresentada no algoritmo 5 consiste no fato de que, ao contrário do método tradicional (proposto no algoritmo 4) que extrai informações da trajetória de apenas uma cidade para inferir parâmetros, utilizamos informações de $M$ cidades. 


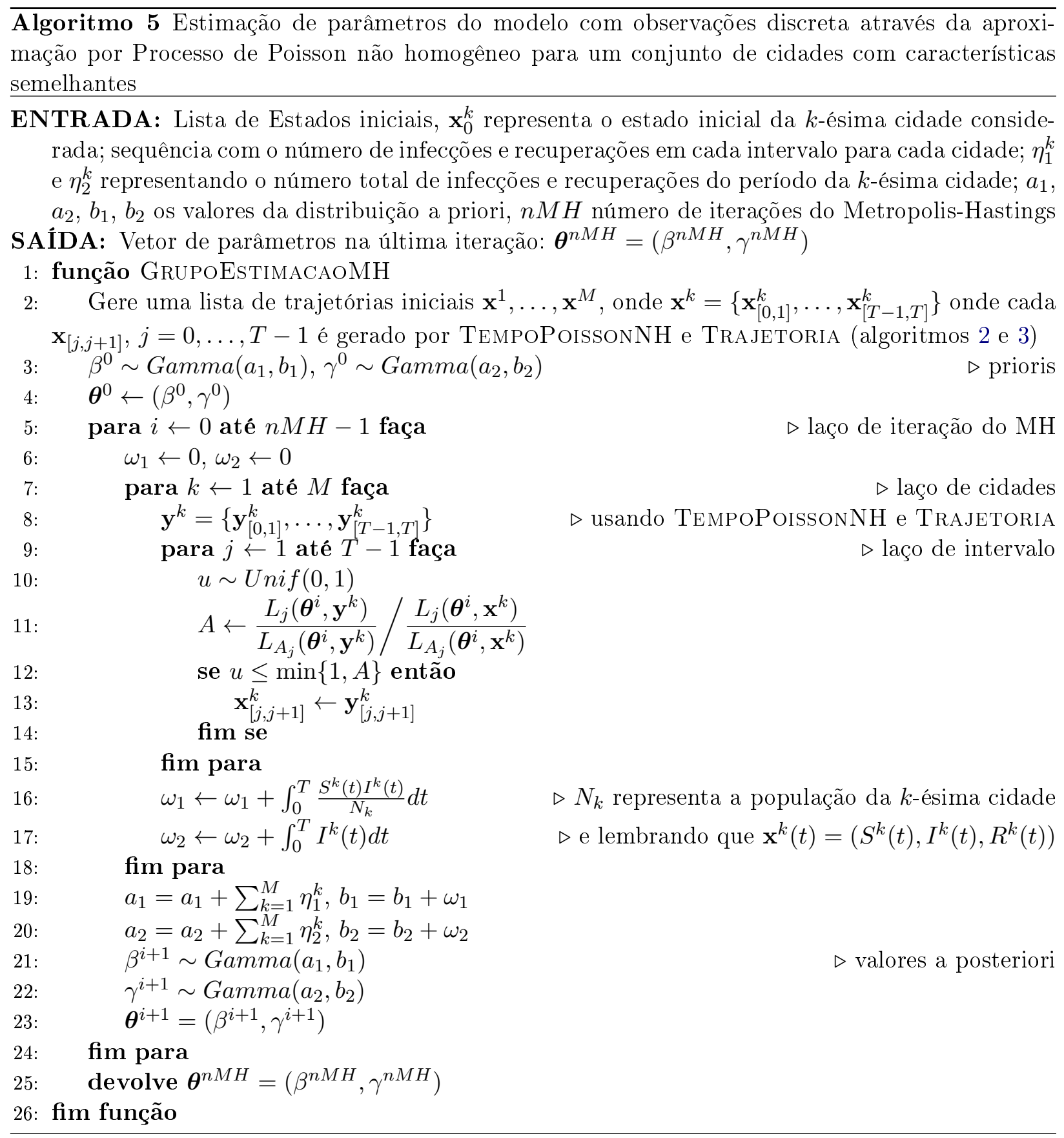




\section{Capítulo 4}

\section{Resultados Obtidos}

De posse do modelo de Gillespie, que descreve a evolução do sistema estocasticamente e das ferramentas descritas no capítulo 3 para a estimação dos parâmetros necessários, estamos aptos a realizar as primeiras simulações. O objetivo desse capítulo é apresentá-los, discutindo brevemente os resultados obtidos.

A densidade demográfica é um fator que possivelmente impacta nos parâmetros do modelo. A aglomeração de pessoas em uma determinada área propicia um número maior de contatos entre as pessoas. Por esse motivo, para a estimação de parâmetros, a característica escolhida para agrupar as cidades foi a densidade demográfica.

\subsection{Estimando parâmetros para grupos de cidades selecionados atra- vés do perfil demográfico}

Nessa abordagem foram construídos três grupos de acordo com o perfil demográfico das cidades. Estimativas populacionais e demográficas aqui adotadas foram baseadas no Censo de 2010, disponível na página do IBGE [IBG10].

\subsubsection{Grupo 1: Cidades com densidade demográfica superior à $1000 \mathrm{hab} / \mathrm{km}^{2}$}

Primeiramente foram selecionados os municípios com densidade demográfica superior à 1000 $h a b / \mathrm{km}^{2}$.

Notemos que em algumas localidades, apesar de haver o registro de ocorrência da doença, o número de infectados não evolui para um surto epidêmico. A figura 4.1 , por exemplo, retrata o número de notificações no município de Queimados. Notemos que o número de casos oscila durante 6 a 8 semanas, sempre abaixo de 10 notificações até atingir o seu máximo de 30 na semana 201312. Após o ponto de máximo, observa-se observa-se uma rápida redução até a aparente extinção da doença no período.

Observando os gráficos de notificação de casos de dengue para cada uma dessas cidades, podemos selecionar aquelas em que a infecção passou do regime letárgico (sem um número expressivo de notificações verificadas) para o regime de crescimento acelerado de infectados. Essas cidades selecionadas serão reunidas no grupo 1.

A tabela 4.1 apresenta as cidades com maior densidade

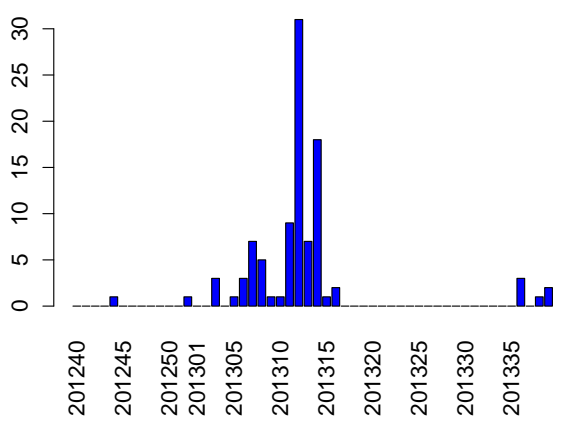

Figura 4.1: Notificações do município de Queimados. Notamos que a infecção não evolui para um regime epidêmico demográfica no estado do Rio de Janeiro.

Analisando os gráficos de novas infecções projetadas (conforme descrito na seção 3.3) do grupo 1 na figura 4.2, selecionamos para cada cidade a semana inicial da infecção, ou seja, o momento 


\begin{tabular}{lccc}
\hline Cidade & Área $\left(\mathrm{km}^{2}\right)$ & População & densidade demográfica $\left(\mathrm{hab} / \mathrm{km}^{2}\right)$ \\
\hline \hline São João de Meriti & 35.216 & 458673 & 13024.56 \\
Nilópolis & 19.393 & 157425 & 8117.62 \\
Belford Roxo & 77.815 & 469332 & 6031.38 \\
Rio de Janeiro & 1200.279 & 6320446 & 5265.81 \\
Mesquita & 39.062 & 168376 & 4310.48 \\
São Gonçalo & 247.709 & 999728 & 4035.9 \\
Niterói & 133.916 & 487562 & 3640.8 \\
Duque de Caxias & 467.619 & 855048 & 1828.51 \\
Queimados & 75.695 & 137962 & 1822.6 \\
Nova Iguaçu & 521.247 & 796257 & 1527.6 \\
Volta Redonda & 182.483 & 257803 & 1412.75 \\
Japeri & 81.871 & 95492 & 1166.37 \\
\hline
\end{tabular}

Tabela 4.1: Municipios com densidade demográfica superior a $1000 \mathrm{hab} / \mathrm{km}^{2}$ segundo o Censo 2010 [IBG10]. Em destaque os municipios do grupo 1

em que o regime letárgico da doença é quebrado. Tomando as observações da semana inicial e das quatro semanas subsequentes, obtemos para cada município o período de ajuste de cinco semanas, apresentado na figura 4.2 .

Para o grupo 1, composto pelas oito cidades citadas anteriormente, definindo o período de ajuste de cinco semanas e fixando o parâmetro $\mu=3$ que relaciona o número de infectados sintomáticos e assintomáticos $\left(I_{\text {proj }}=(1+\mu) I_{s}\right)$, conforme a hipótese 2.3.2 proposta, vamos analisar os resultados obtidos. A informação completa sobre a configuração do cenário de estimação de parâmetros está disponível na tabela 4.2 .

\begin{tabular}{c|c|r}
\hline descrição & parâmetro & valor \\
\hline \hline parâmetro que relaciona infectados sintomáticos e assintomáticos & $\mu$ & 3 \\
\hline \multirow{3}{*}{ parâmetros para a distribuição a priori } & $a_{1}$ & 1.01 \\
\cline { 2 - 3 } & $a_{2}$ & 1.01 \\
\cline { 2 - 3 } & $b_{1}$ & 1 \\
\cline { 2 - 3 } & $b_{2}$ & 1 \\
\hline número de iterações do Metropolis-Hastings & $n M H$ & 100 \\
\hline número de semanas observadas no ajuste & $n$ SemAjuste & 5 \\
\hline
\end{tabular}

Tabela 4.2: Configuração do cenário para estimação de parâmetros do grupo 1

Apresentamos na figura 4.3 a evolução das estimativas do parâmetros do modelo ao longo das 100 iterações programadas do passo de Metropolis-Hastings. Na figura 4.3a temos as estimativas para $\beta$ e em 4.3b, as estimativas pra $\gamma$. Em ambas as figuras, no quadro em destaque, observamos as últimas 30 iterações para cada parâmetro.

Notamos que, a partir do ponto determinada pela distribuição a priori, há uma rápida convergência para valores nas proximidades de $(\beta, \gamma)=(1.13,0.88)$, mantendo-se nessas cercanias com relativa estabilidade até o final da execução.

Conforme podemos observar na tabela 4.2, foram fixados valores iguais para as distribuições à priori para ambos os parâmetros do modelo. Tais valores foram selecionados visando aumentar a probabilidade da estimativa a priori se manter no intervalo $[0,2]$.

A figura 4.4a apresenta a distribuição a priori para os parâmetros. Já as distribuições para $\beta$ e $\gamma$ podem ser conferidas nas figuras $4.4 \mathrm{~b}$ e $4.4 \mathrm{c}$, respectivamente.

Com base na distribuição a posteriori, podemos encontrar um intervalo de confiança para cada um dos parâmetros. Adotando o nível de confiança de 95\%, obtemos: 


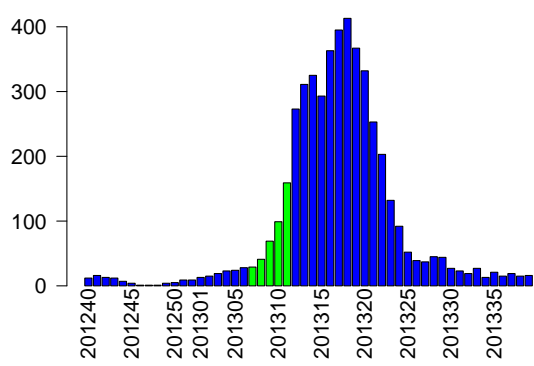

(a) Belford Roxo

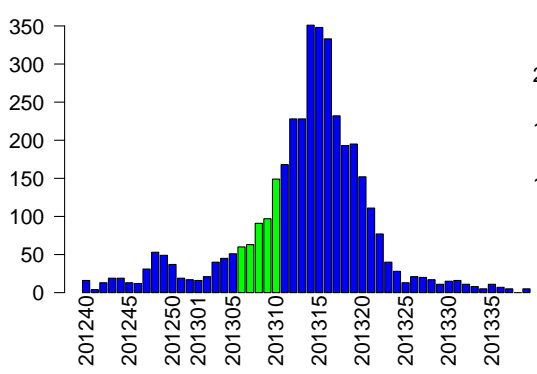

(d) Nova Iguaçu

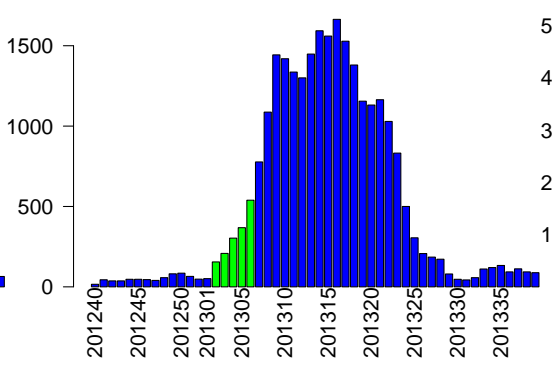

(b) Duque de Caxias

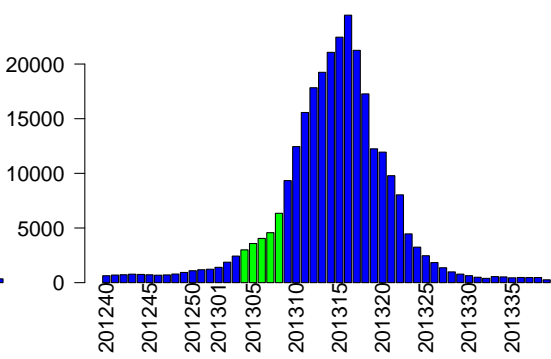

(e) Rio de Janeiro

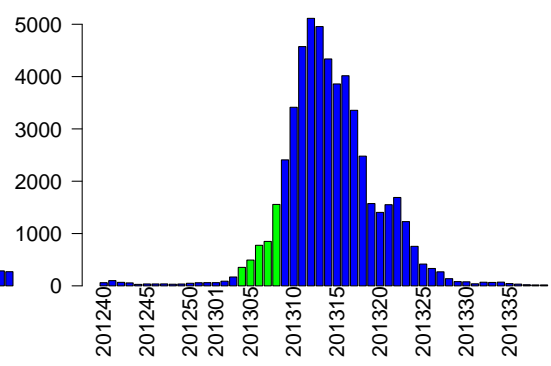

(c) Niterói

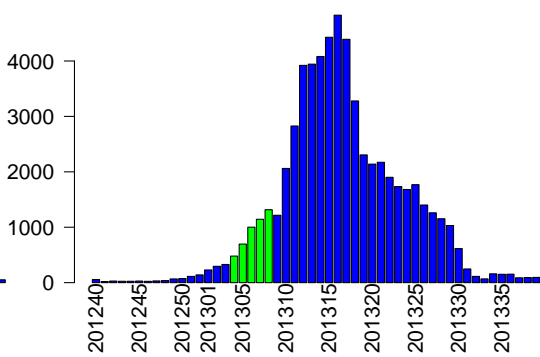

(f) São Gonçalo

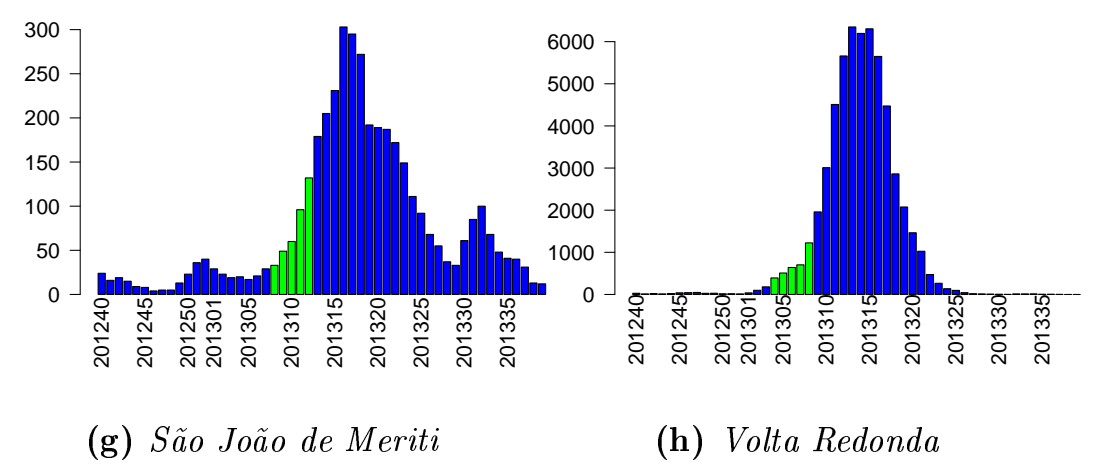

Figura 4.2: Número de infectados projetados (suavizados) para a dengue por semana nas cidades do grupo 1 no período 2012-2013. As semanas do periodo ajuste escolhidas são destacadas na cor verde

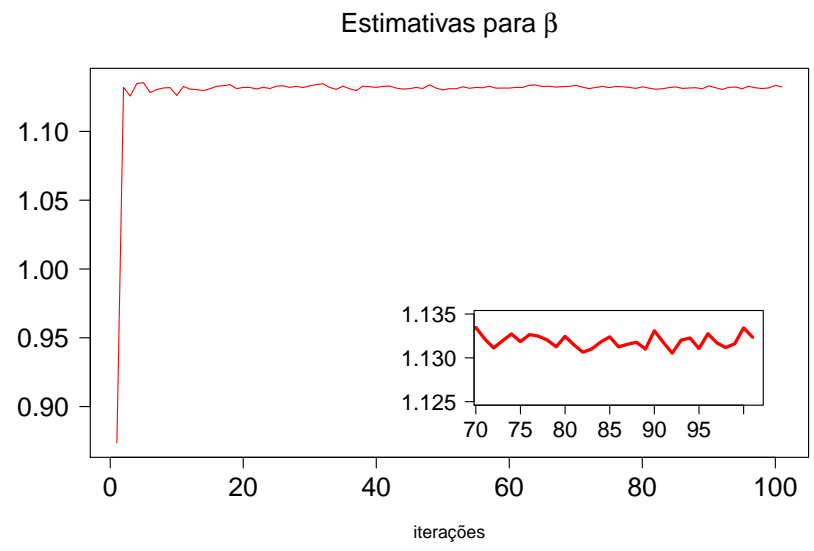

(a) Estimativas para $\beta$ para o Grupo 1
Estimativas para $\gamma$

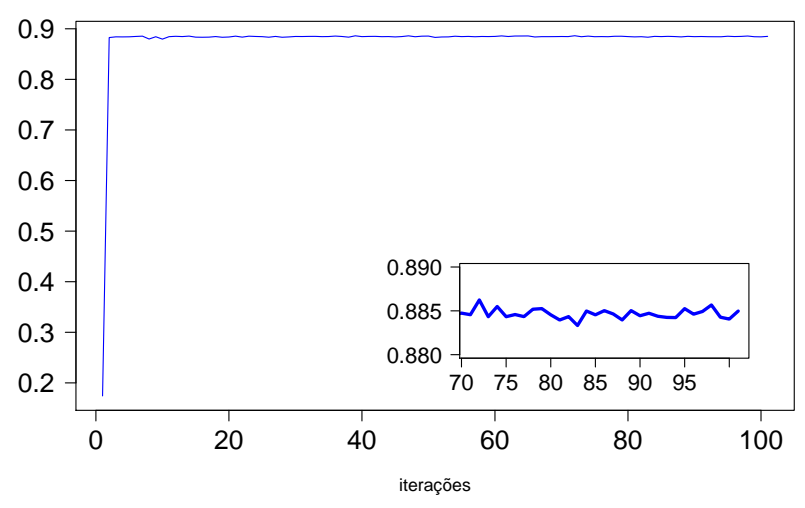

(b) Estimativas para $\gamma$ para o Grupo 1

Figura 4.3: Evolução das estimativas ao longo das iterações de Metropolis-Hastings para os parâmetros para o Grupo 1. Em destaque as últimas 30 iterações do Metropolis-Hastings 
Priori de $\beta$ e $\gamma$

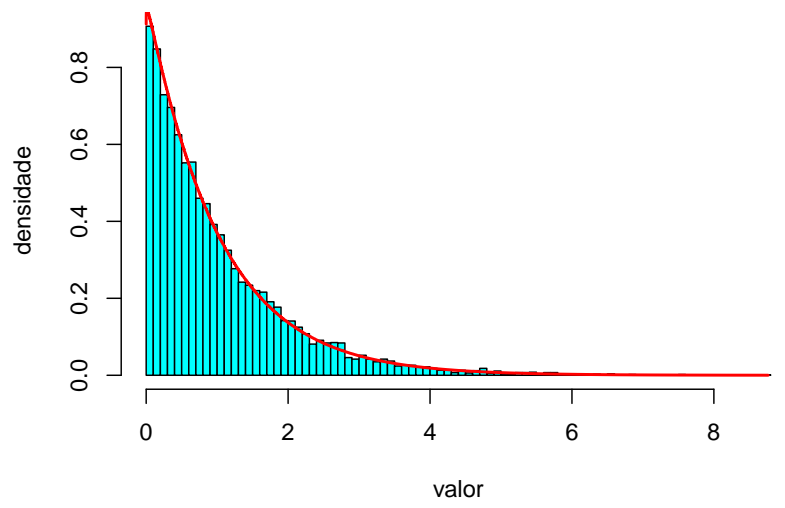

(a) Distribuição a priori para $\beta$ e $\gamma$

Posteriori de $\beta$

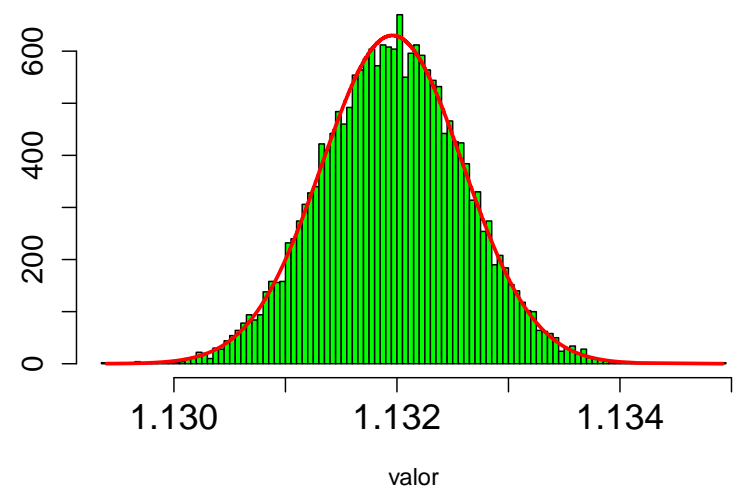

(b) Distribuição a posteriori para $\beta$ para as cidades do Grupo 1
Posteriori de $\gamma$

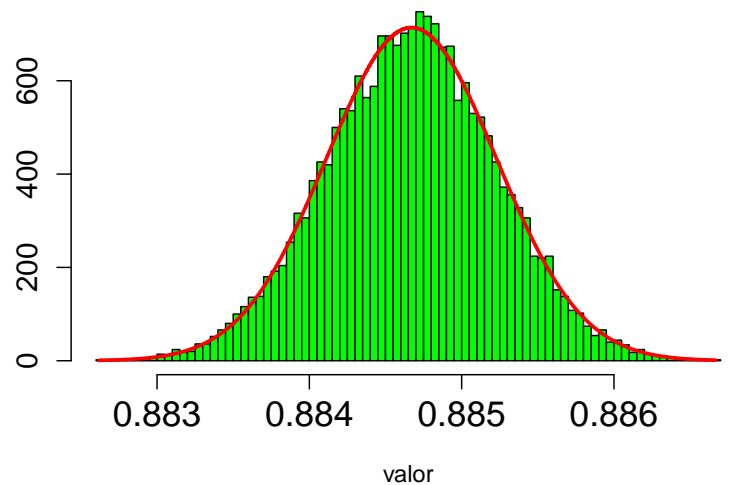

(c) Distribuição a posteriori para $\gamma$ para as cidades do Grupo 1

Figura 4.4: Distribuições a priori e posteriori para os parâmetros do modelo no Grupo 1

$$
\begin{aligned}
I C(\beta, 0.95) & =[1.130719,1.133201] \\
I C(\gamma, 0.95) & =[0.8835754,0.8857661]
\end{aligned}
$$

Tomando valores nesse intervalo como estimadores para nossos parâmetros, tornamos-nos aptos a realizar simulações do algoritmo de Gillespie. Os estimadores escolhidos em nossas simulações são: $\hat{\beta}_{g_{1}}=1.132$ e $\hat{\gamma}_{g_{1}}=0.8847$.

Até o presente momento, o procedimento adotado consistiu em determinar um instante em que o sistema entra em um regime de crescimento do número de infecções para uma cidade (ou um grupo de cidades) e dessa forma definir o período de ajuste com a finalidade de extrair estimativas para os parâmetros do modelo. Vamos agora exibir uma possível aplicação do modelo. A ideia é, utilizando os parâmetros estimados no período de ajuste, observar qual é a projeção fornecida pelo modelo para as semanas seguintes ao período de ajuste.

Por tratar-se de um modelo estocástico, somente a repetição recorrente do algoritmo pode fornecer a tendência do sistema ao longo do tempo. Por esse motivo, foram geradas cem trajetórias descrevendo o cenário futuro após a última semana de ajuste.

A informação fornecida por essas simulações foi resumida segundo uma metodologia semelhante à conhecida como boxplot funcional, cujos detalhes podem ser obtidos no artigo de Sun [SG09]. 


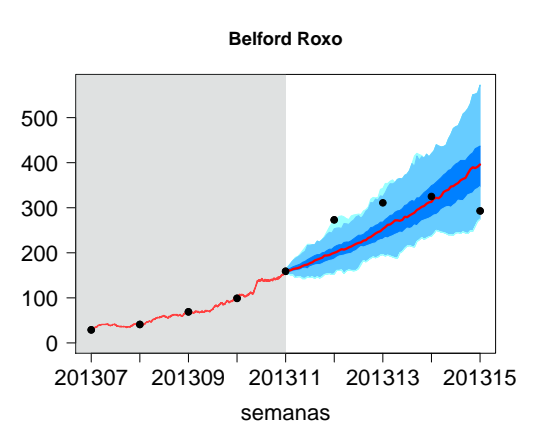

(a) Belford Roxo

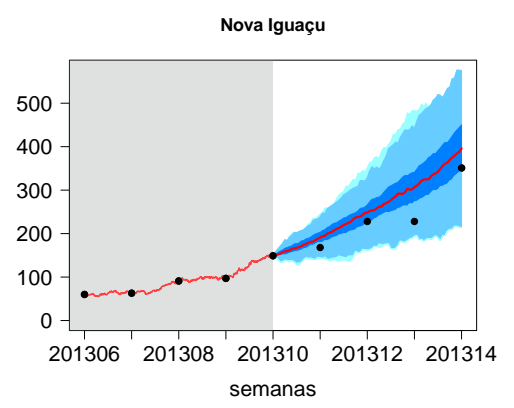

(d) Nova Iguaçu

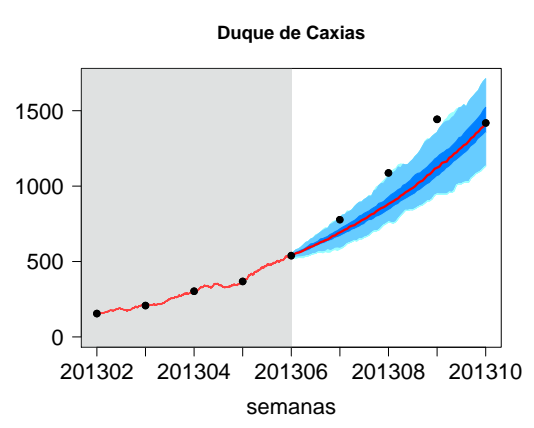

(b) Duque de Caxias

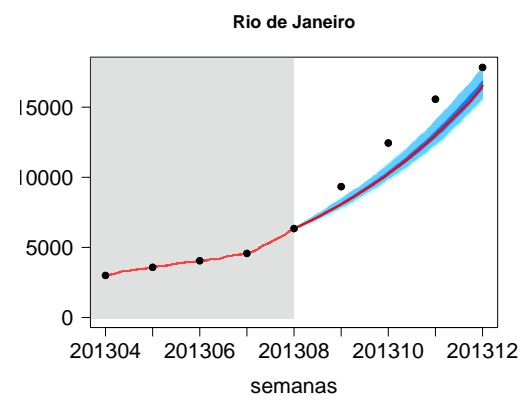

(e) Rio de Janeiro

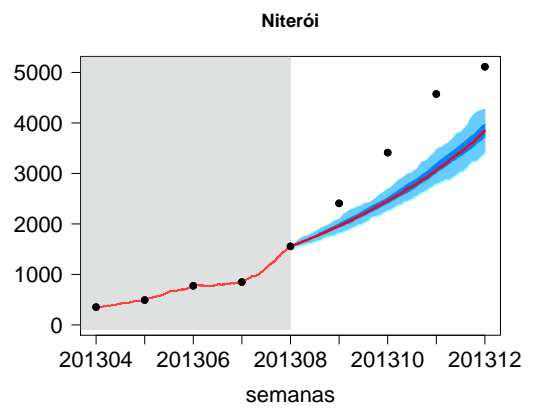

(c) Niterói

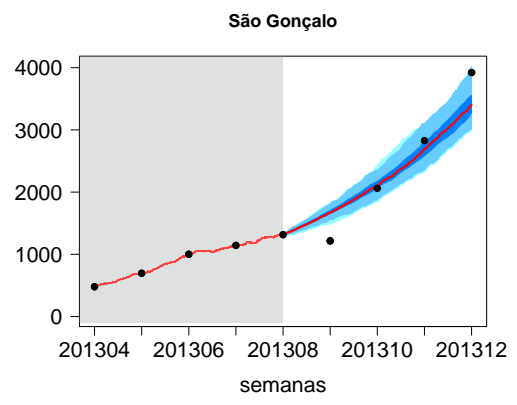

(f) São Gonçalo

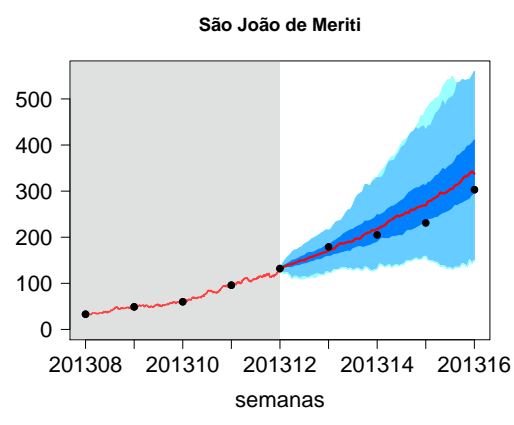

(g) São João de Meriti

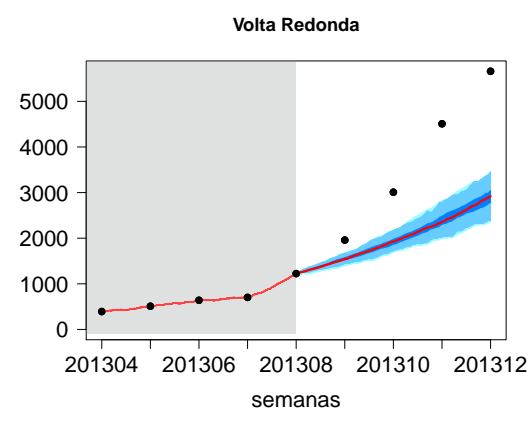

(h) Volta Redonda

Figura 4.5: Projeções geradas pela simulação do algoritmo de Gillespie para as cidades do grupo 1 para as semanas seguintes ao periodo de ajuste. Os parâmetros adotados foram: $\hat{\beta}_{g_{1}}=1.132$ e $\hat{\gamma}_{g_{1}}=0.8847$

A figura 4.5 apresenta a projeção para as quatro semanas seguintes ao período de ajuste. Cada gráfico pode ser decomposto em duas partes: o período de ajuste é identificado com o fundo em cinza. Nesse setor, a curva em vermelho representa a última trajetória obtida pelo método de MetropolisHastins (a curva utilizada na estimação de parâmetros segundo a distribuição a posteriori).

Já para a segunda parte, são apresentadas sob a forma de boxplots funcionais o resumo das informações obtidas pela simulação de cem trajetórias descrevendo cenários futuros através do algoritmo de Gillespie. Graficamente, esse resumo pode ser decomposto na curva em vermelho e faixas com diferentes tonalidades de azul. A trajetória em vermelho representa a evolução da mediana ao longo do tempo, a faixa em azul mais escuro que envolve a curva vermelha, representa o intervalo entre quartis de um boxplot. A próxima camada imediatamente externa, representada por uma tonalidade levemente mais clara que a anterior, representa as caudas do boxplot: os limitantes inferior e superior dessa faixa são dados por $Q_{1}-1.5\left(Q_{3}-Q_{1}\right)$ e $Q_{3}+1.5\left(Q_{3}-Q_{1}\right)$, respectivamente. Aqui, $Q_{1}$ e $Q_{3}$ representam os primeiro e terceiro quartis. Por fim, na tonalidade mais clara de azul utilizada, temos a representação dos chamados outliers, pontos externos à camada restrita pelas caudas do boxplot.

Analisando agora os dados obtidos através das simulações, notemos primeiramente que as cida- 
des em que a infecção atinge um número maior de pessoas, possuem menor variabilidade entre as trajetórias simuladas, sobretudo nas cidades de Rio de Janeiro, Niterói, São Gonçalo e Volta Redonda. Isso pode ser explicado analisando as expressões das funções de propensidade: quanto menor o número de infectados do sistema, maior a sensibilidade em relação à um evento de infecção.

Notemos que para a cidade de São Gonçalo (figura 4.5f) e Duque de Caxias (figura 4.5b) as projeções das trajetóras futuras ajustam de forma satisfatória o comportamento das próximas quatro semanas. Já para a cidade do Rio de Janeiro (figura 4.5e), embora o número de infectados seja subestimado, e os pontos observados estejam fora das faixas projetadas pelo boxplot funcional, temos que ao menos o comportamento do sistema é capturado pelo modelo.

Já para as cidades de Belford Roxo (figura 4.5a), Nova Iguaçu (figura 4.5d) e São João de Meriti (figura $4.5 \mathrm{~g}$ ), temos uma grande variabilidade das trajetórias simuladas, entretanto, a projeção pode ser considerada satisfatória.

Por outro lado, notamos que a previsão obtida para as cidades Niterói e Volta Redonda (figuras $4.5 \mathrm{c}$ e $4.5 \mathrm{~h}$, respectivamente) subestimam consideravelmente os pontos posteriormente observados.

\subsubsection{Grupo 2: Cidades com densidade demográfica entre 200 e $1000 \mathrm{hab} / \mathrm{km}^{2}$}

Apresentamos agora na tabela 4.3 as cidades que possuem densidade demográfica entre 200 e 1000 habitantes por $\mathrm{km}^{2}$. Novamente filtramos as cidades sem relevância epidemiológica, apresentando uma quantidade irrisória de infectados, como por exemplo, a cidade de Paracambi, que durante o período de estudo apresentou apenas duas notificações de casos de dengue. Vamos ainda destacar o caso das cidades de Maricá e São Pedro da Aldeia que, embora apresentem uma quantidade significativa de infectados possuem um comportamento atípico na evolução do número de notificações.

\begin{tabular}{lccc}
\hline Cidade & Área $\left(\mathrm{km}^{2}\right)$ & População & densidade demográfica $\left(\mathrm{hab} / \mathrm{km}^{2}\right)$ \\
\hline \hline Magé & 388.496 & 227322 & 585.13 \\
Itaboraí & 430.373 & 218008 & 506.56 \\
Rio das Ostras & 229.043 & 105676 & 461.38 \\
Cabo Frio & 410.415 & 186227 & 453.75 \\
Iguaba Grande & 51.945 & 22851 & 439.91 \\
Itaguaí & 275.867 & 109091 & 395.45 \\
Armação dos Búzios & 70.278 & 27560 & 392.16 \\
Petrópolis & 795.798 & 295917 & 371.85 \\
Maricá & 362.571 & 127461 & 351.55 \\
Porto Real & 50.748 & 16592 & 326.95 \\
Barra Mansa & 547.226 & 177813 & 324.94 \\
Pinheiral & 76.53 & 22719 & 296.86 \\
Seropédica & 283.762 & 78186 & 275.53 \\
São Pedro da Aldeia & 332.792 & 87875 & 264.05 \\
Paracambi & 179.68 & 47124 & 262.27 \\
Três Rios & 326.135 & 77432 & 237.42 \\
Teresópolis & 770.601 & 163746 & 212.49 \\
Tanguá & 145.503 & 30732 & 211.21 \\
Saquarema & 353.566 & 74234 & 209.96 \\
Angra dos Reis & 825.088 & 169511 & 205.45 \\
\hline
\end{tabular}

Tabela 4.3: Municípios com densidade demográfica entre 200 e $1000 \mathrm{hab} / \mathrm{km}^{2}$ segundo o Censo 2010 [IBG10]. Em destaque os municípios do grupo 2

A figura 4.6 apresenta o número de infectados da cidade de Maricá ao longo do período de estudo. Podemos observar que o número de notificações oscila do início do período analisado na 
semana 201240 até 201249 sem potencial de evoluir para um regime epidêmico. Após um breve momento de aparente inatividade em humanos do agente infeccioso, notamos seu reaparecimento na semana 201301 e um rápido crescimento até um patamar superior ao observado anteriormente (entre 100 e 200 infectados), mas ao contrário do esperado, o número de notificações mantém-se com pequenas oscilações até a semana 201307.

Nas três semanas subsequentes notamos uma explosão no número de infectados atingindo o ápice em 201310, quando presenciamos a barreira das 600 infecções ser rom-

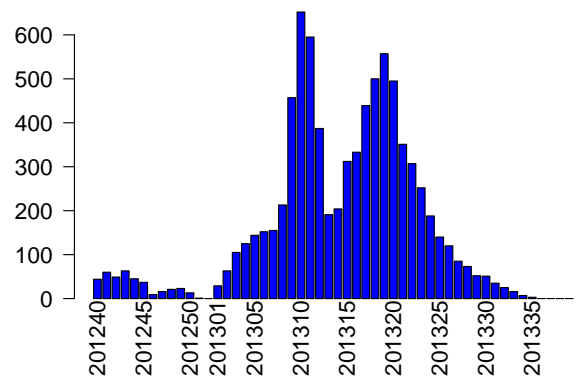

Figura 4.6: Comportamento atípico no número de infectados no município de $M a-$ ricá

considerar as cidades de Maricá e São Pedro da Aldeia (que apresenta comportamento semelhante) na inferência dos parâmetros.

A evolução do número de infectados projetados pelas notificações registradas em cada uma das cidades selecionadas no grupo 2 estão apresentadas na figura 4.7 .

A evolução das estimativas dos parâmetros ao longo dos 100 passos de Metropolis-Hastings pode ser conferida na figura 4.8 .

Utilizamos nesse grupo a mesma distribuição a priori adotada no grupo 1, cuja densidade pode ser observada na figura 4.4a. A distribuição a posteriori obtida pode ser conferida observando a figura 4.9

Através das distribuições a posteriori para $\beta$ e $\gamma$, propomos intervalos de confiança para os parâmetros:

$$
\begin{aligned}
I C(\beta, 0.95) & =[1.174446,1.179089] \\
I C(\gamma, 0.95) & =[0.8541983,0.8581431]
\end{aligned}
$$

Adotando valores centrais nesses intervalos como estimativas para os parâmetros, $\hat{\beta}_{g_{2}}=1.177$ e $\hat{\gamma}_{g_{2}}=0.8567$, podemos simular o comportamento dos sistemas nas cidades do grupo 2 nas semanas seguintes ao período de ajuste. A figura 4.10 apresenta os dados resumidos dos cenários projetados pelo modelo para as próximas semanas.

Notemos que o modelo ajusta o comportamento futuro das cidades de Cabo Frio e Saquarema (figuras $4.10 \mathrm{c}$ e $4.10 \mathrm{~h}$ ) de forma bastante precisa. Observamos ainda que a mediana das trajetórias simuladas se aproxima do número de infectados nas quatro primeiras semanas que sucedem o período de ajuste em Itaguaí (figura 4.10e) e nas três semanas em Magé (figura 4.10f). Após esse período a precisão é comprometida pela interrupção do crescimento do número de infectados observados nas duas cidades. Essa queda do número de infectados também é observada nas cidades Barra Mansa, Seropédica e Teresópolis (figuras 4.10b, 4.10i e 4.10j, respectivamente). Notamos que, apesar da característica oscilatória das observações nessas cidades, a maioria dos pontos está confinada nas faixas determinadas pelo boxplot funcional.

Já em Angra dos Reis observamos que o número de infecções é superestimado durante as três 


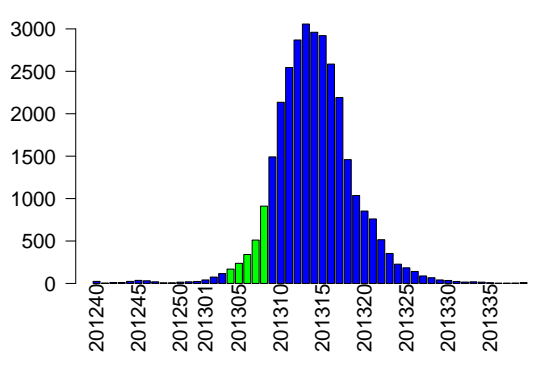

(a) Angra dos Reis

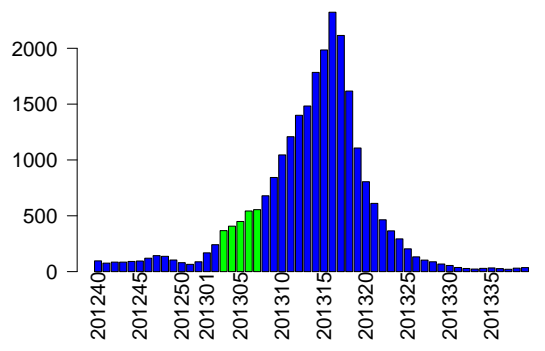

(d) Itaborai

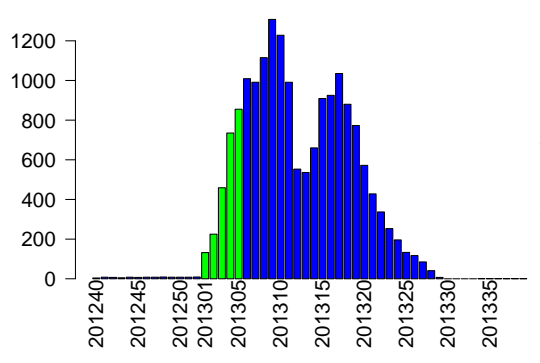

(g) Rio das Ostras

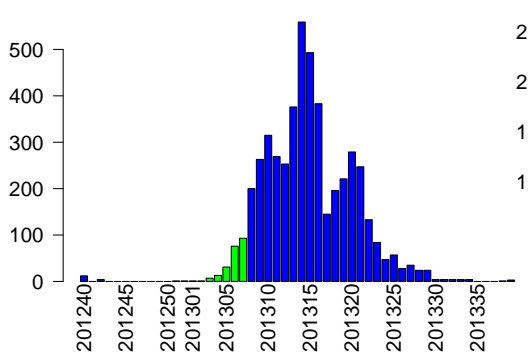

(b) Barra Mansa

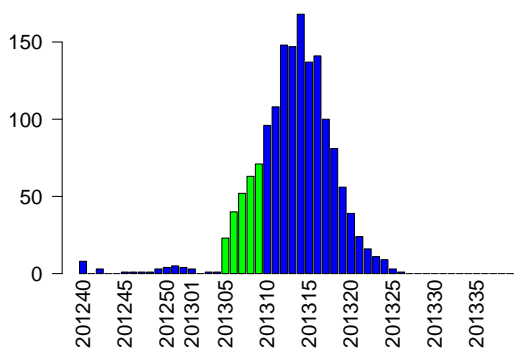

(e) Itaguaí

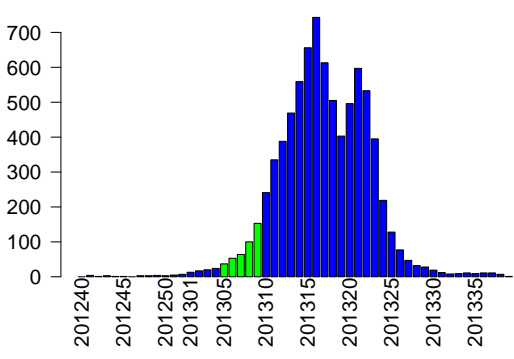

(h) Saquarema

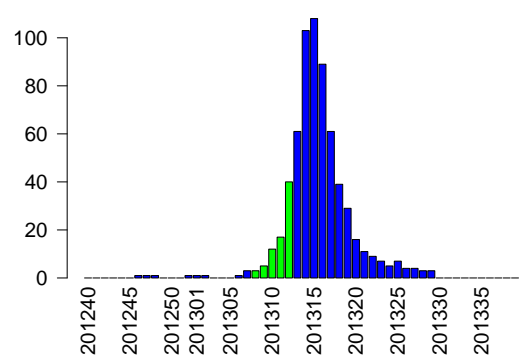

(j) Teresópolis

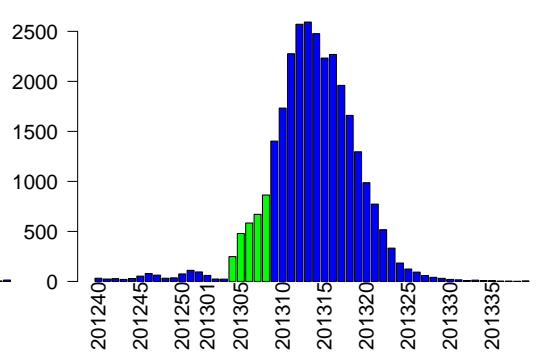

(c) Cabo Frio

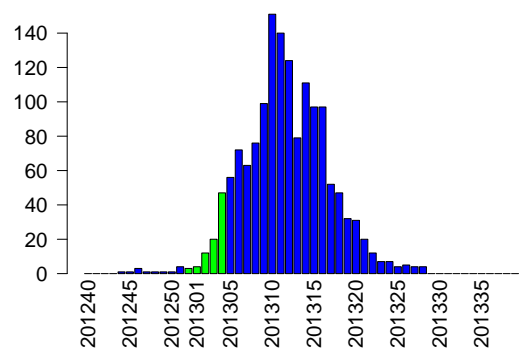

(f) Magé

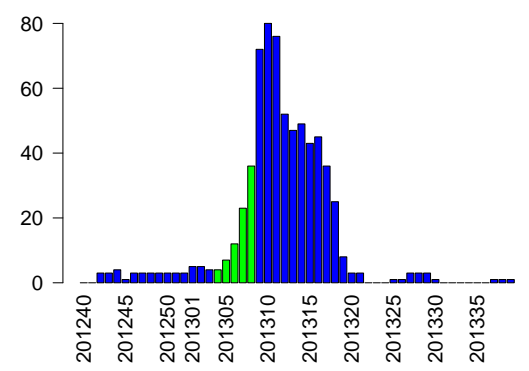

(i) Seropédica

Figura 4.7: Número de infectados projetados (suavizados) para a dengue por semana nas cidades do grupo 2 no período 2012-2013. As semanas do período ajuste escolhidas são destacadas na cor verde

semanas subsequentes ao período de ajuste, mas notamos que a tendência de crescimento é, de certa forma capturada, culminando na convergência da previsão até a observação na semana 201312. Em Itaboraí, pelo contrário, observamos uma subestimação dos infectados. A tendência de crescimento do sistema é capturado, porém sem a mesma precisão do caso de Angra dos Reis.

O único caso em que as previsões divergem significativamente das observações é na cidade de Rio das Ostras, como pode ser observado na figura 4.10g. Observando a série de infectados de Rio das Ostras (figura $4.7 \mathrm{~g}$ ) notamos que a doença demonstra um crescimento acelerado logo nas primeiras semanas, perdendo força logo após o período de ajuste. Concluímos então que a dinâmica da doença 
Estimativas para $\beta$

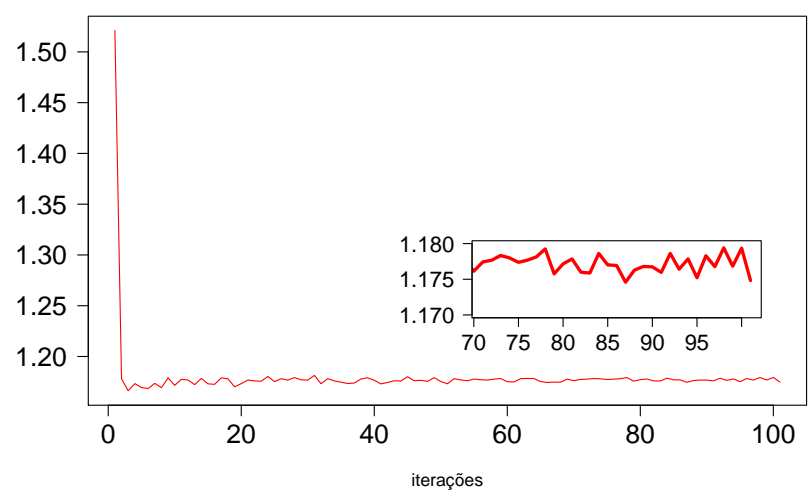

(a) Estimativas para $\beta$ para o Grupo 2

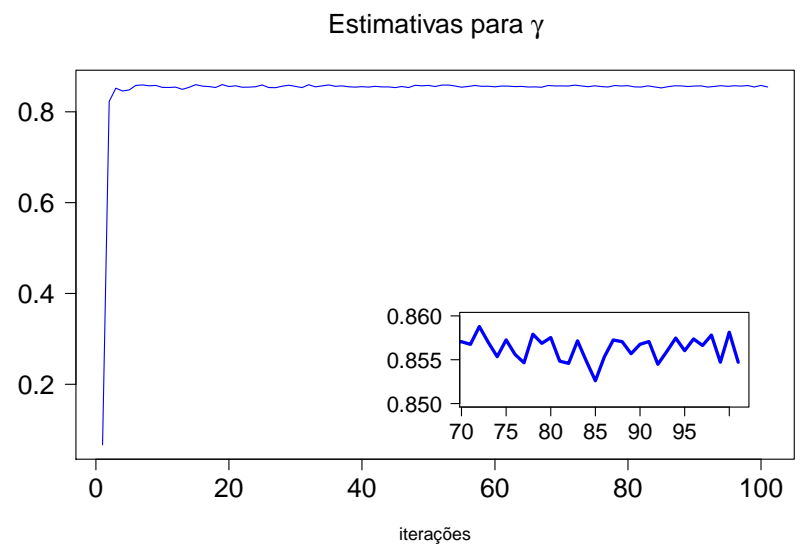

(b) Estimativas para $\gamma$ para o Grupo 2

Figura 4.8: Estimativas para os parâmetros para o Grupo 2. Em destaque as últimas 30 iterações do Metropolis-Hastings

Posteriori de $\beta$

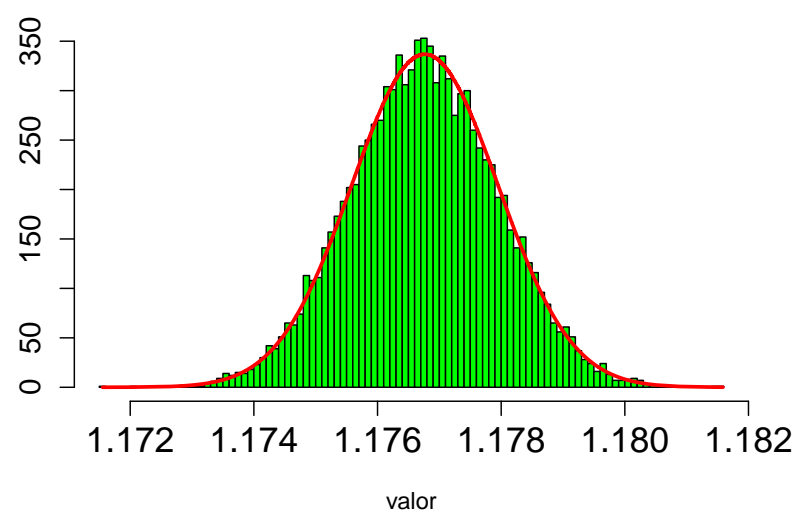

(a) Distribuição a posteriori para $\beta$ para as cidades do Grupo 2
Posteriori de $\gamma$

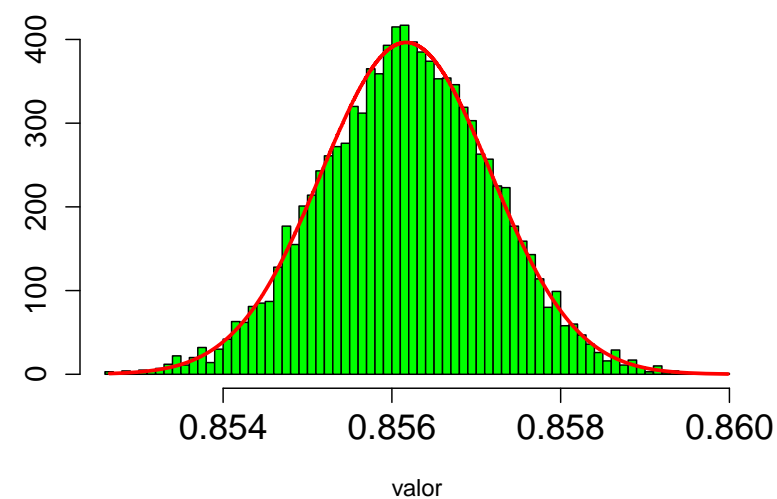

(b) Distribuição a posteriori para $\gamma$ para as cidades do Grupo 2

Figura 4.9: Distribuições a priori e posteriori para os parâmetros do modelo no Grupo 2

sofreu uma alteração nesse período, o que pode explicar a divergência da previsão. 


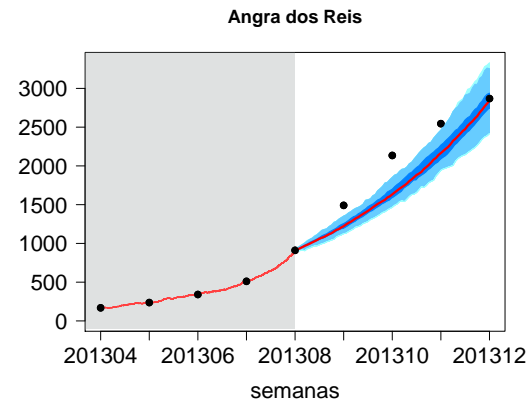

(a) Angra dos Reis

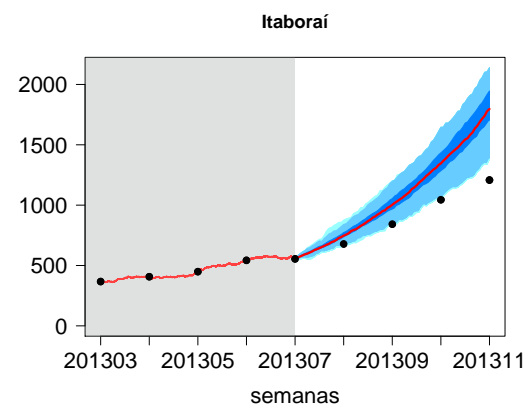

(d) Itaborai

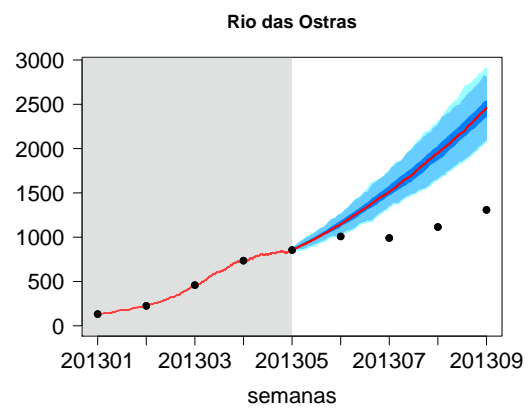

(g) Rio das Ostras

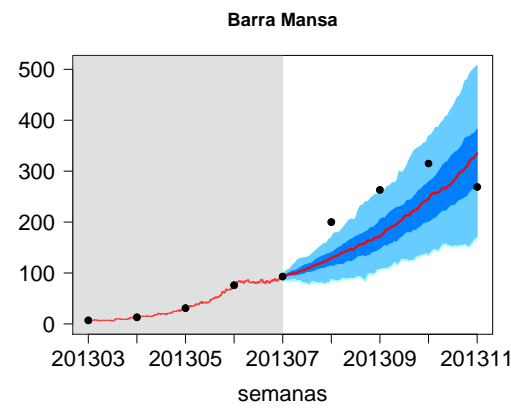

(b) Barra Mansa

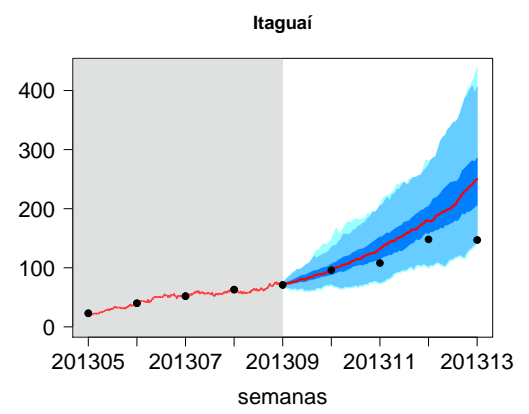

(e) Itaguai

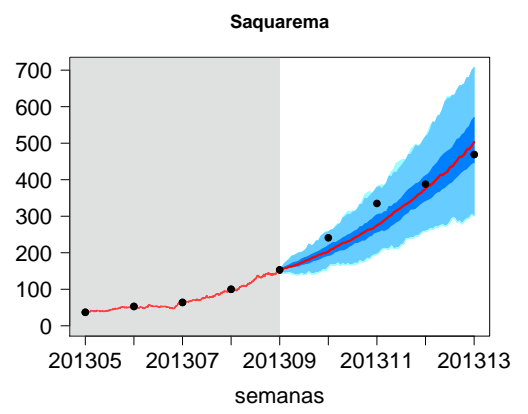

(h) Saquarema

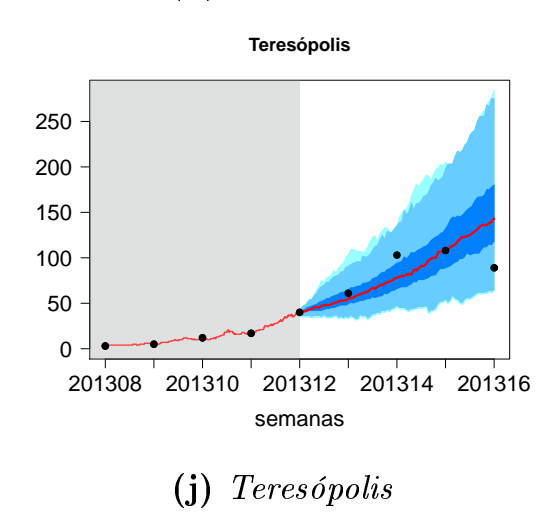

(j) Teresópolis

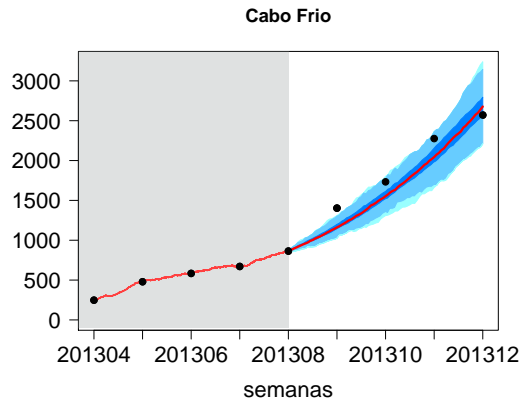

(c) Cabo Frio

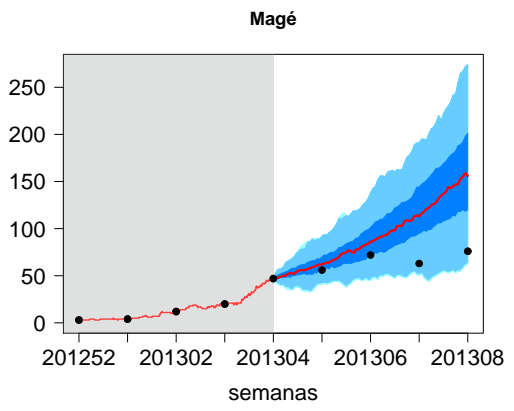

(f) Magé

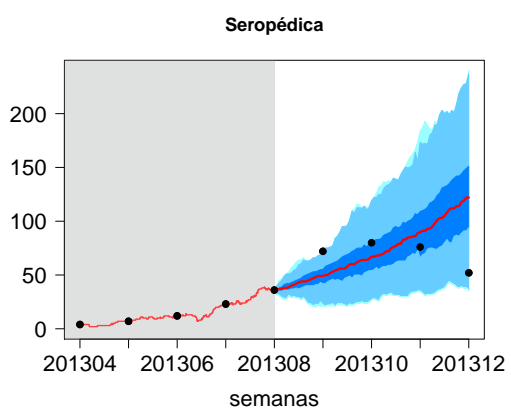

(i) Seropédica

Figura 4.10: Projeções geradas pela simulação do algoritmo de Gillespie para as cidades do grupo 2 para as semanas seguintes ao periodo de ajuste. Os parâmetros adotados foram: $\hat{\beta}_{g_{2}}=1.177$ e $\hat{\gamma}_{g_{2}}=0.8567$

\subsubsection{Grupo 3: Cidades com densidade demográfica abaixo de $200 \mathrm{hab} / \mathrm{km}^{2}$}

Vamos finalmente analisar o último grupo, composto pelos municípios com densidade demográfica abaixo de $200 \mathrm{hab} / \mathrm{km}^{2}$. As cidades cuja infecção se mostrou relevante e foram, por este motivo, escolhidas para integrar o grupo 3 estão destacadas na tabela 4.4 . 


\begin{tabular}{|c|c|c|c|}
\hline Cidade & Área $\left(k^{2}\right)$ & População & densidade demográfica $\left(h a b / \mathrm{km}^{2}\right)$ \\
\hline Nova Friburgo & 933.414 & 182082 & 195.07 \\
\hline Mendes & 97.035 & 17935 & 184.83 \\
\hline Araruama & 638.023 & 112008 & 175.55 \\
\hline Arraial do Cabo & 160.286 & 27715 & 172.91 \\
\hline Macaé & 1216.845 & 206728 & 169.89 \\
\hline Barra do Piraí & 578.965 & 94778 & 163.7 \\
\hline Guapimirim & 360.766 & 51483 & 142.7 \\
\hline Rio Bonito & 456.455 & 55551 & 121.7 \\
\hline Itatiaia & 245.146 & 28783 & 117.41 \\
\hline Campos dos Goytacazes & 4026.712 & 463731 & 115.16 \\
\hline Resende & 1095.254 & 119769 & 109.35 \\
\hline Aperibé & 94.635 & 10213 & 107.92 \\
\hline Mangaratiba & 353.083 & 36456 & 103.25 \\
\hline Areal & 110.919 & 11423 & 102.99 \\
\hline Engenheiro Paulo de Frontin & 132.936 & 13237 & 99.57 \\
\hline São José do Vale do Rio Preto & 220.432 & 20251 & 91.87 \\
\hline Itaperuna & 1105.341 & 95841 & 86.71 \\
\hline Miguel Pereira & 289.183 & 24642 & 85.21 \\
\hline Paty do Alferes & 318.801 & 26359 & 82.68 \\
\hline Casimiro de Abreu & 460.773 & 35347 & 76.71 \\
\hline Comendador Levy Gasparian & 106.888 & 8180 & 76.53 \\
\hline São João da Barra & 455.044 & 32747 & 71.96 \\
\hline Macuco & 77.719 & 5269 & 67.8 \\
\hline Santo Antônio de Pádua & 603.355 & 40589 & 67.27 \\
\hline Bom Jardim & 384.639 & 25333 & 65.86 \\
\hline Vassouras & 538.134 & 34410 & 63.94 \\
\hline Conceição de Macabu & 347.272 & 21211 & 61.08 \\
\hline Bom Jesus do Itabapoana & 598.824 & 35411 & 59.13 \\
\hline Porciúncula & 302.024 & 17760 & 58.8 \\
\hline Cachoeiras de Macacu & 953.801 & 54273 & 56.9 \\
\hline Valença & 1304.813 & 71843 & 55.06 \\
\hline Carmo & 322.415 & 17434 & 54.07 \\
\hline Itaocara & 431.335 & 22899 & 53.09 \\
\hline Piraí & 505.374 & 26314 & 52.07 \\
\hline Italva & 293.82 & 14063 & 47.86 \\
\hline Quatis & 286.093 & 12793 & 44.72 \\
\hline Paraty & 925.053 & 37533 & 40.57 \\
\hline Natividade & 386.74 & 15082 & 39 \\
\hline São Francisco de Itabapoana & 1122.437 & 41354 & 36.84 \\
\hline São Fidélis & 1031.558 & 37543 & 36.39 \\
\hline Sapucaia & 541.239 & 17525 & 32.38 \\
\hline Laje do Muriaé & 249.974 & 7487 & 29.95 \\
\hline Quissamã & 712.852 & 20242 & 28.4 \\
\hline São José de Ubá & 250.28 & 7003 & 27.98 \\
\hline Cantagalo & 749.278 & 19830 & 26.47 \\
\hline Cambuci & 561.698 & 14827 & 26.4 \\
\hline Silva Jardim & 937.547 & 21349 & 22.77 \\
\hline São Sebastião do Alto & 397.897 & 8895 & 22.36 \\
\hline Rio Claro & 840.59 & 17425 & 20.73 \\
\hline Rio das Flores & 478.312 & 8561 & 17.9 \\
\hline
\end{tabular}

Tabela 4.4: Municípios com densidade demográfica abaixo de $200 \mathrm{hab} / \mathrm{km}^{2}$ segundo o Censo 2010 [IBG10]. Em destaque os municipios do grupo 3

Os gráficos representando o número de infectados ao longo do tempo (já suavizados por aplicação de média móvel de três semanas) nas cidades do grupo 3 são apresentados na figura 4.11.

A seleção das cidades que compõem o grupo 3 foi executada através da avaliação do número de notificações de casos de dengue no período que compreende a semana 40 de 2012 até a semana 39 de 2013. Notemos, no entanto, um fato interessante: ao tomarmos a tabela 4.4 e selecionarmos 


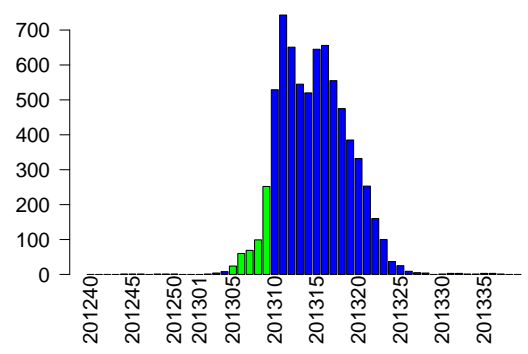

(a) Araruama

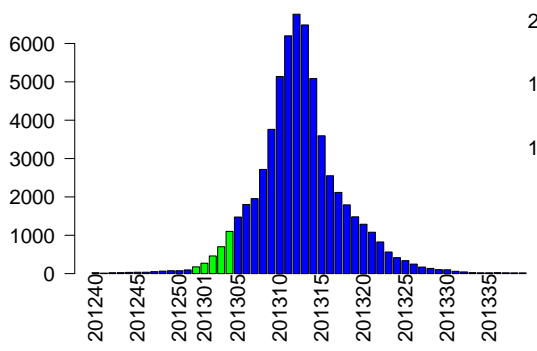

(d) Campos dos Goytacazes

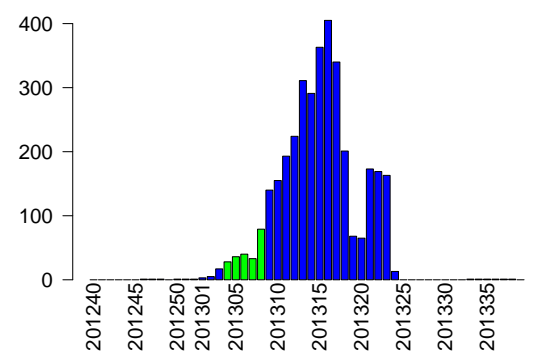

(g) Nova Friburgo

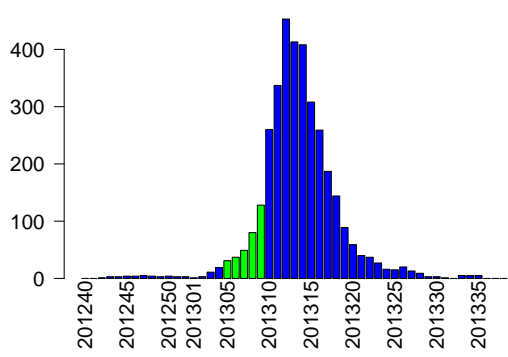

(b) Barra do Piraí

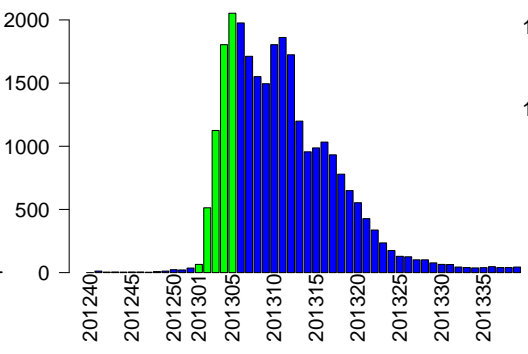

(e) Itaperuna

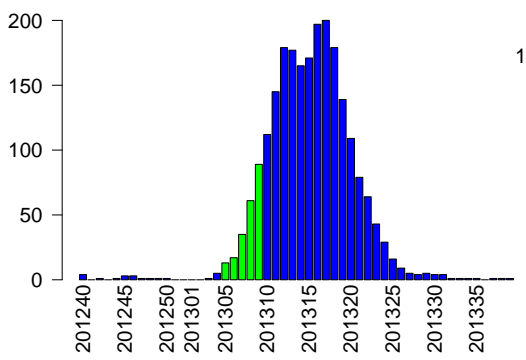

(h) Resende

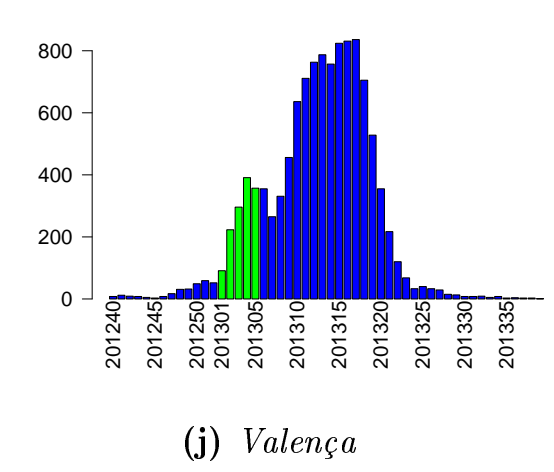

(j) Valença

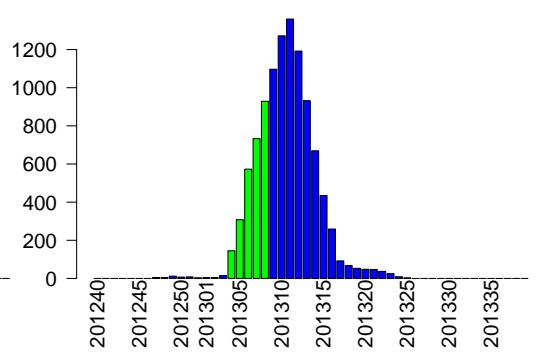

(c) Cachoeiras de Macacu

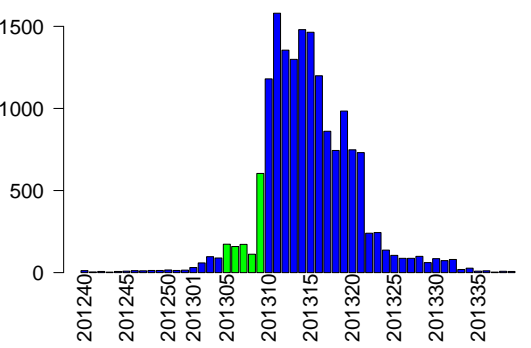

(f) Macaé

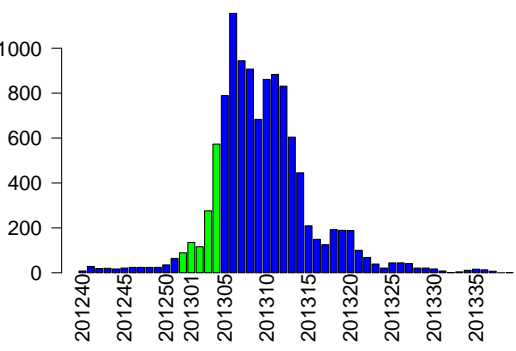

(i) Rio Bonito

Figura 4.11: Número de infectados projetados (suavizados) para a dengue por semana nas cidades do grupo 3 no periodo 2012-2013. As semanas do período ajuste escolhidas são destacadas na cor verde

os municípios que possuem população superior a 54000 habitantes, obteremos as mesmas cidades escolhidas para compor o grupo 3. Esse fato revela que há uma profunda relação entre o tamanho populacional e a ocorrência de um surto notável de dengue.

Por esse motivo, apesar da extensa lista de municípios apresentada na tabela 4.4, temos selecionados no grupo 3 apenas dez cidades: Araruama, Barra do Piraí, Cachoeiras de Macacu, Campos dos Goytacazes, Itaperuna, Macaé, Nova Friburgo, Resende, Rio Bonito e Valença.

Utilizando as informações do período de ajuste para estimar os parâmetros, observamos agora, na figura 4.12, a rápida convergência dos valores das estimativas dos parâmetros ao longo das 
iterações do método de Metropolis-Hastings.

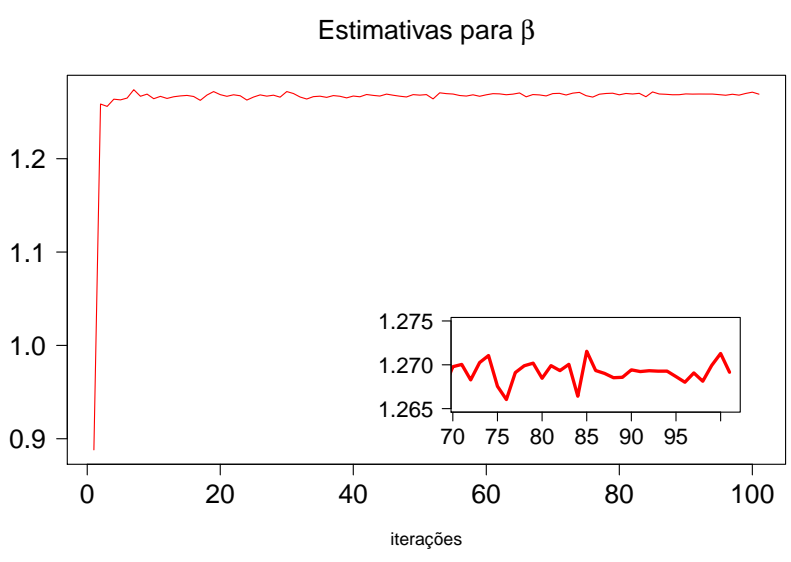

(a) Estimativas para $\beta$ para o Grupo 3

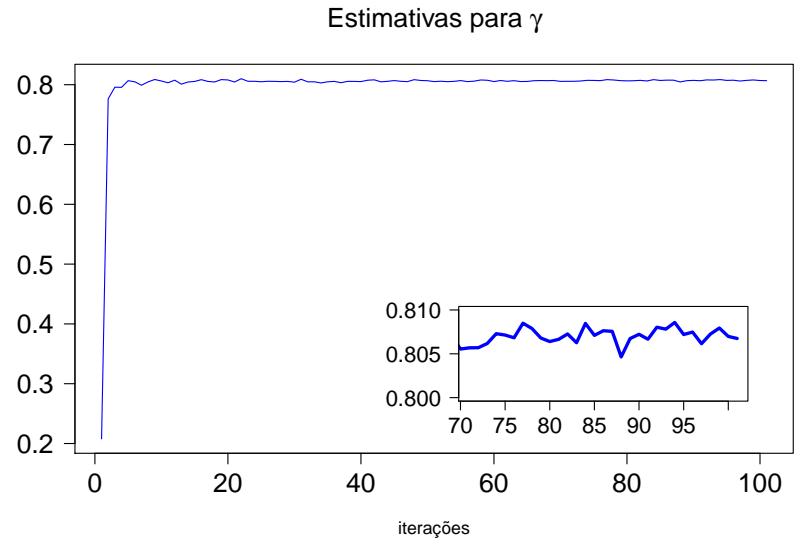

(b) Estimativas para $\gamma$ para o Grupo 3

Figura 4.12: Estimativas para os parâmetros para o Grupo 3. Em destaque as últimas 30 iterações do Metropolis-Hastings

Utilizando a mesma distribuição a priori dos grupos anteriores (que pode ser conferida na figura 4.4a), obtemos a distribuição a posteriori dos parâmetros, exibida na figura 4.13.

Posteriori de $\beta$

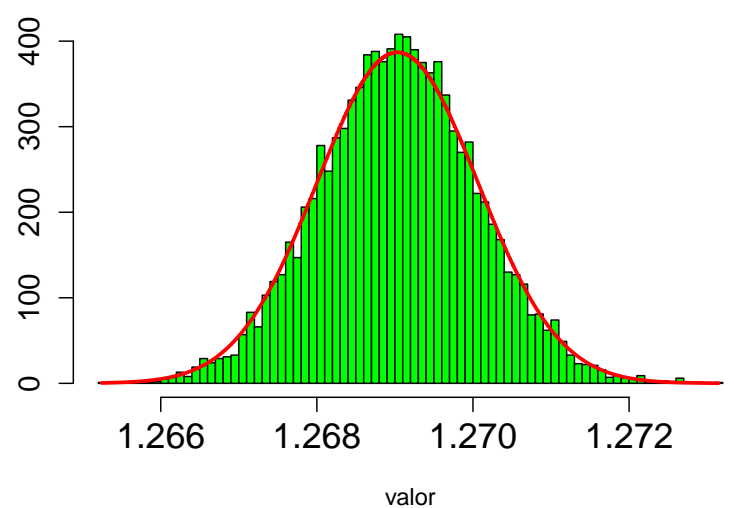

(a) Distribuição a posteriori para $\beta$ para as cidades do Grupo 3
Posteriori de $\gamma$

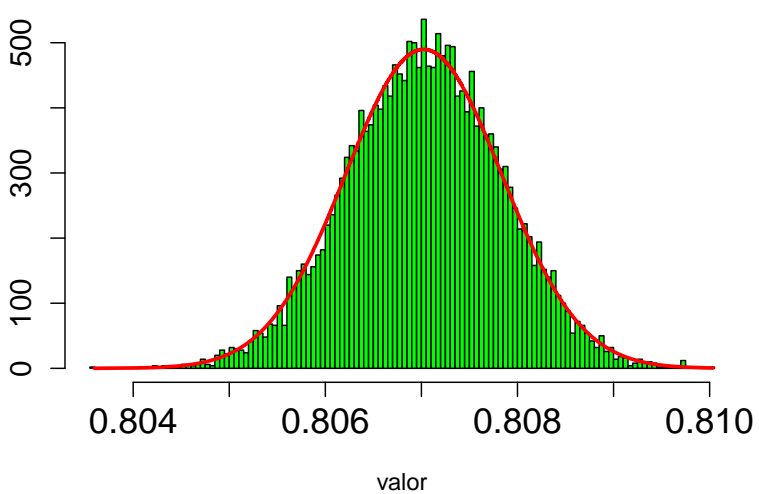

(b) Distribuição a posteriori para $\gamma$ para as cidades do Grupo 3

Figura 4.13: Distribuições a priori e posteriori para os parâmetros do modelo no Grupo 3

Através da distribuição a posteriori, definimos um intervalo de confiança para os parâmetros:

$$
\begin{aligned}
I C(\beta, 0.95) & =[1.267014,1.271055] \\
I C(\gamma, 0.95) & =[0.805428,0.8086205]
\end{aligned}
$$

Baseados nos intervalos de confiança dos parâmetros, definimos os estimadores: $\hat{\beta}_{g_{3}}=1.269$ e $\hat{\gamma}_{g_{3}}=0.807$. Assim como procedemos nos grupos anteriores, vamos tentar agora extrapolar o comportamento do sistema para as semanas seguintes ao período de ajuste. O resumo das trajetórias simuladas são apresentadas na figura 4.14 .

Analisando os resultados obtidos, notamos que o modelo encontra uma dificuldade maior em tentar prever o comportamento futuro. Em muitos casos a tendência crescente é interrompida, ocasionando em uma divergência entre a previsão do modelo e o valor observado. 


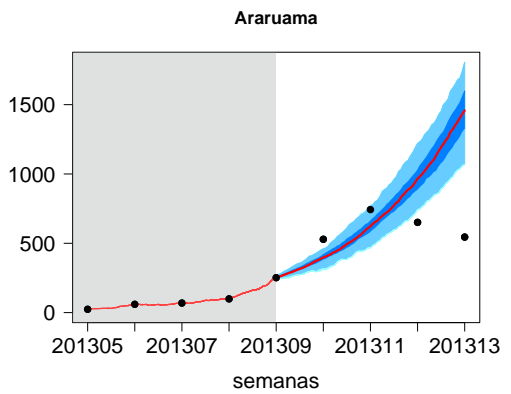

(a) Araruama

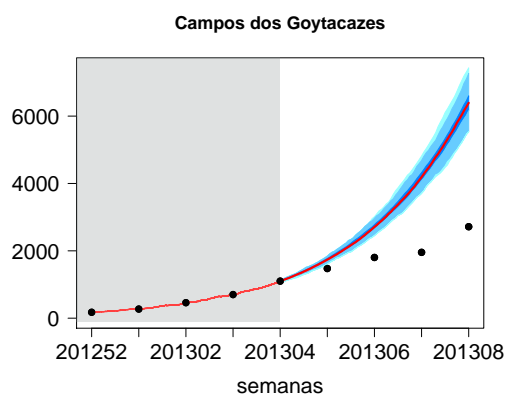

(d) Campos dos Goytacazes

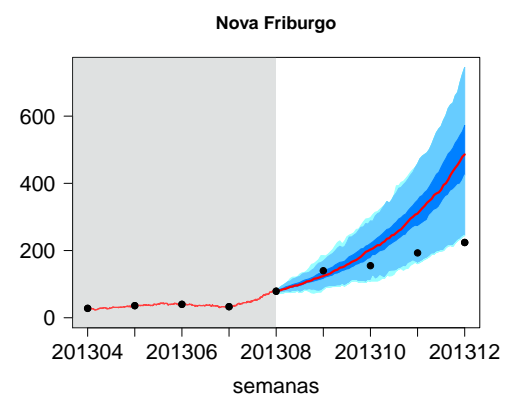

(g) Nova Friburgo

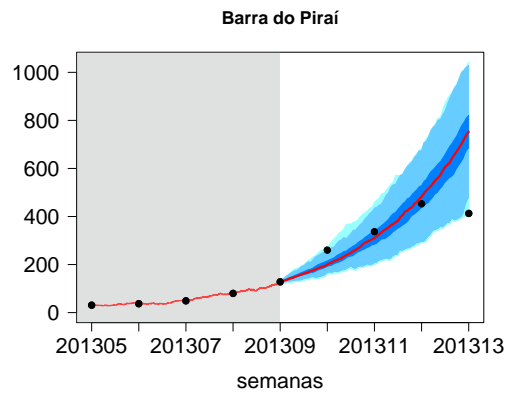

(b) Barra de Piraí

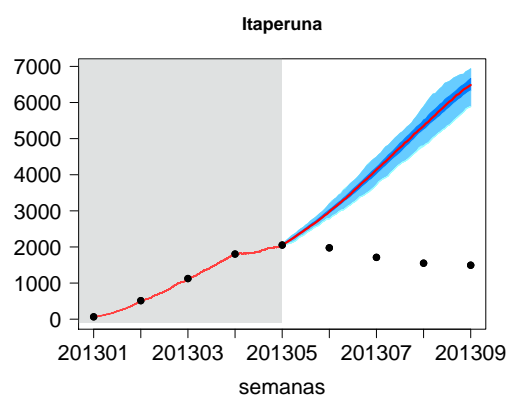

(e) Itaperuna

Resende

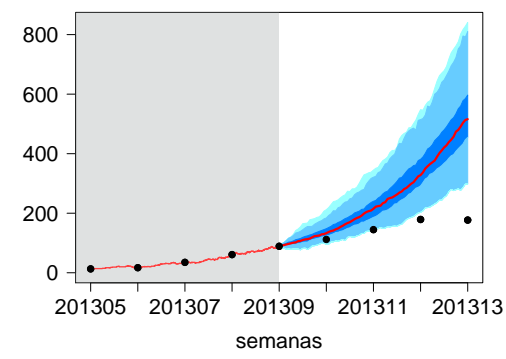

(h) Resende

Valença

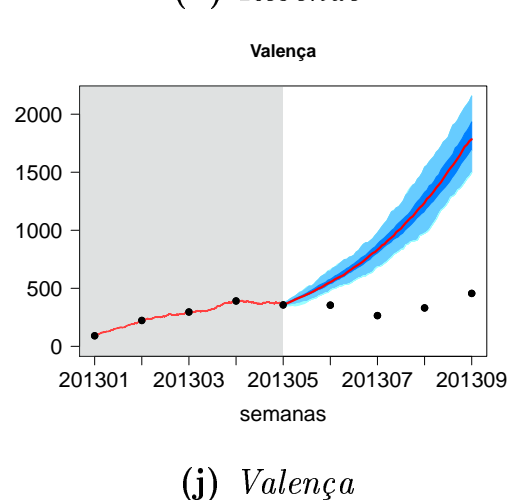

(j) Valença

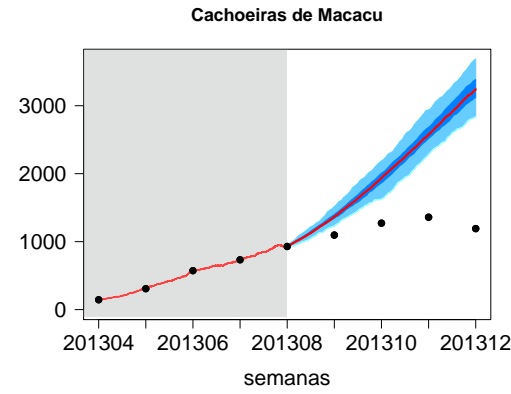

(c) Cachoeiras de Macacu

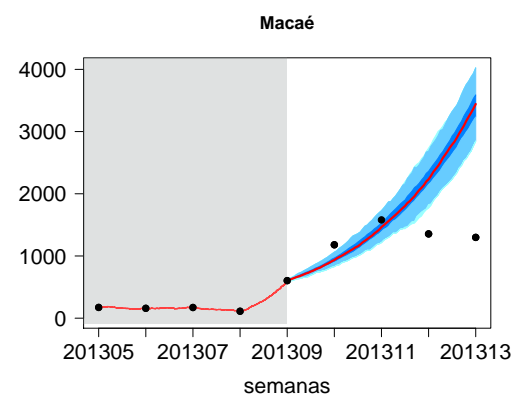

(f) Macaé

Rio Bonito

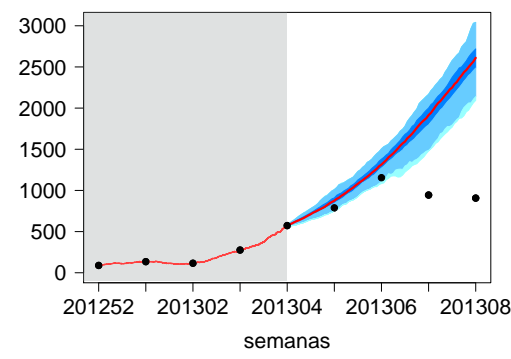

(i) Rio Bonito

Figura 4.14: Projeções geradas pela simulação do algoritmo de Gillespie para as cidades do grupo 3 para as semanas seguintes ao periodo de ajuste. Os parâmetros adotados foram: $\hat{\beta}_{g_{3}}=1.269$ e $\hat{\gamma}_{g_{3}}=0.807$

Um dos melhores ajustes pode ser observado nas cidade de Barra do Piraí (figura 4.14b) em que o modelo captura de forma bastante acurada o comportamento das três semanas seguintes ao período de ajuste. Podemos destacar ainda os gráficos obtidos para os municípios de Araruama, Macaé e Rio Bonito (figuras 4.14a, 4.14f e 4.14i, respectivamente) em que é obtida uma previsão satisfatória para as duas primeiras semanas apenas. Em todas essas cidades, notamos que há uma quebra no regime de crescimento do número de infectados, que não é capturado pelo modelo. 
Já para as cidades de Nova Friburgo e Resende, embora tenhamos um regime crescente do número de infecções, notamos que a projeção obtida pelo modelo para o número de infectados se aproxima de forma razoável apenas nas duas primeiras semanas do período de previsão. Após esse período o modelo superestima o número de infectados. Outra cidade cujo modelo superestima as infecções é Campos dos Goytacazes. De fato, essa cidade aparenta possuir uma dinâmica diferente das observadas em outras cidades.

Ao analisarmos a figura 4.11e observamos que o pico do número de infectados no período 201240 a 201339 da cidade de Itaperuna coincide com a última semana do período de ajuste. Nas semanas seguintes, observa-se que o número de infectados apresenta pequenas oscilações e começa a ter trajetória decrescente após algumas semanas.

Em Cachoeiras de Macacu (figura 4.11c), notamos que a doença apresenta um crescimento acelerado: da semana inicial até o pico da curva de infectados são decorridos apenas sete semanas. É bastante provável então, que no período de previsão do modelo o regime de crescimento acelerado que caracteriza o início de infecção (e é o foco do nosso estudo) já tenha sido alterado. Essa é uma hipótese para a superestimação observada nas simulações.

Por fim, temos a cidade de Valença. Recorrendo novamente à série de infectados (figura 4.11j) notemos que após o período de ajuste, ocorre uma interrupção no regime crescente de infecções: o número de infectados oscila entre 300 a 350 infectados, para depois retomar o ritmo de crescimento.

Antes de terminar essa seção, vamos resumir as informações obtidas nos testes. A tabela apresenta os parâmetros estimados para cada grupo:

\begin{tabular}{c|c|c|c|c}
\hline Grupo & número de cidades & densidade demográfica & $\hat{\beta}$ & $\hat{\gamma}$ \\
\hline 1 & 8 & acima de $1000 \mathrm{hab} / \mathrm{km}^{2}$ & 1.132 & 0.8847 \\
2 & 10 & entre 200 e $1000 \mathrm{hab} / \mathrm{km}^{2}$ & 1.177 & 0.8567 \\
3 & 10 & abaixo de $200 \mathrm{hab} / \mathrm{km}^{2}$ & 1.269 & 0.807 \\
\hline
\end{tabular}

Tabela 4.5: Resumo dos estimadores encontrados

A figura 4.15 exibe a localização das cidades de cada grupo.

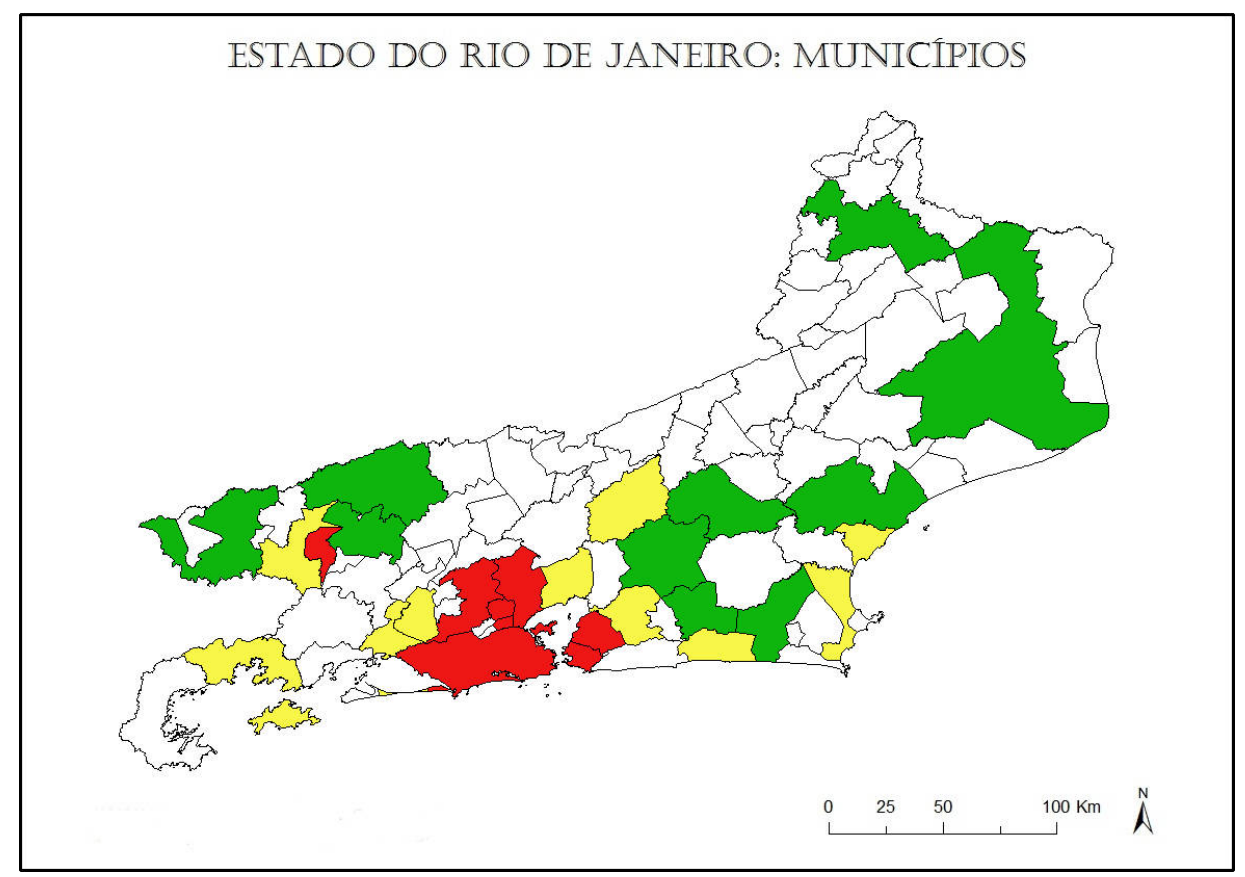

Figura 4.15: Localização das cidades de cada grupo no estado do Rio de Janeiro. As cidades do grupo 1, 2 e 3 são identificadas pelas cores vermelho, amarelo e verde, respectivamente

Podemos notar que, com exceção à Volta Redonda, as demais cidades do grupo 1 são todas pertencentes à região Metropolitana do Rio de Janeiro, que é tipicamente a região mais populosa 
do estado.

Já os demais grupos possuem as cidades distribuídas de forma mais dispersa ao longo do estado. Podemos, no entanto observar que as cidades do grupo 2 concentram-se mais na faixa centro sul do estado, com destaque para as proximidades das cidades pertencentes ao grupo 1 .

Notamos que o grupo 3, por sua vez, pode ser subdividido em três subgrupos: a região nortenoroeste do estado representado pelas cidades de Campos de Goytacazes, Itaperuna e Macaé, a região fronteiriça entre as regiões dos Lagos e Serrana (Araruama, Cachoeiras de Macacu, Nova Friburgo e Rio Bonito) e o terceiro subgrupo em que as cidades se localizam na região do Médio Paraíba, representadas pelos municípios de Barra do Piraí, Resende e Valença.

Uma discussão que pode ser levantada refere-se à relação entre a qualidade das previsões obtidas e a localização das cidades estudadas em cada grupo. A maior precisão obtida nos ajustes do grupo 1 pode ter sido facilitado pela proximidade geográfica dos municípios do grupo.

Já a dificuldade encontrada em capturar o comportamento das cidades do grupo 3 pode ser decorrente da comparação de três regiões relativamente distantes.

\subsubsection{Testando cenários alternativos}

Como já discutimos, pelo fato de possuirmos apenas o número de notificações semanais de novos casos de dengue, é difícil mensurar a contribuição exercida pelos infectados assintomáticos na dinâmica da doença. A hipótese assumida é a de que para cada notificação (infectado sintomático), hajam três outros casos omitidos em nossa base de dados $(\mu=3)$. Diante da incerteza desse cenário, foram estimados parâmetros para o modelo com diferentes valores de $\mu$. Os resultados obtidos estão resumidos na tabela.

\begin{tabular}{|c|cc|c|c|c|c|}
\multicolumn{1}{l|}{} & \multicolumn{2}{|c|}{ grupo 1 } & \multicolumn{2}{c|}{ grupo 2 } & \multicolumn{2}{c|}{ grupo 3 } \\
& $\hat{\beta}$ & $\hat{\gamma}$ & $\hat{\beta}$ & $\hat{\gamma}$ & $\hat{\beta}$ & $\hat{\gamma}$ \\
\hline$\mu=0$ & 1.131 & 0.8859 & 1.179 & 0.8633 & 1.259 & 0.8120 \\
$\mu=1$ & 1.13 & 0.8847 & 1.176 & 0.8588 & 1.257 & 0.8075 \\
$\mu=2$ & 1.131 & 0.8848 & 1.177 & 0.8578 & 1.266 & 0.8088 \\
$\mu=3$ & 1.132 & 0.8847 & 1.177 & 0.8567 & 1.269 & 0.807 \\
$\mu=4$ & 1.132 & 0.8839 & 1.18 & 0.8566 & 1.279 & 0.8095 \\
$\mu=5$ & 1.132 & 0.8838 & 1.18 & 0.855 & 1.281 & 0.8068 \\
$\mu=10$ & 1.137 & 0.8847 & 1.19 & 0.854 & 1.312 & 0.806 \\
\hline
\end{tabular}

Tabela 4.6: Resumo dos estimadores encontrados para cada valor de $\mu$

Notamos que a variação de $\mu$ praticamente não influencia a estimativa dos parâmetros do modelo. Já analisamos as previsões exibidas para o cenário com $\mu=3 \mathrm{e}$, diante da variação praticamente insignificante dos parâmetros estimados para $\mu \neq 3$, omitiremos os gráficos desses cenários.

Um outro ponto de atenção é a de que, até o presente momento, consideramos a população inteiramente suscetível até o instante inicial $t_{0}$. Entretanto, em doenças como a dengue no estado do Rio de Janeiro, esse cenário é pouco factível com a realidade: em geral há uma parcela da população que, por já ter adquirido previamente a infecção, é imune ao agente infeccioso. Diante da dificuldade de quantificar a proporção dos indivíduos imunes (recuperados) na população total, foram simulados alguns cenários adicionais.

Definimos a quantidade $p_{\text {sus }} \in[0,1]$ que representa a proporção da população que é suscetível antes do instante inicial da infecção $t_{0}$. Baseados nas observações de infectados obtidas no período de ajuste (que independe de $p_{\text {sus }}$ ), inferimos os parâmetros do modelo. A tabela 4.7 reúne os valores dos estimadores para diferentes valores de $p_{\text {sus }}$. Aqui foram fixados cenários com $\mu=3$.

Notamos que o valor estimado para $\beta$ é inversamente proporcional ao parâmetro de controle $p_{\text {sus }}$. Esse comportamento é previsível se observarmos a função de propensidade. Relembrando as funções de propensidade: 


\begin{tabular}{|c|ccc|cc|cc|}
\multicolumn{1}{c|}{} & \multicolumn{2}{|c|}{ grupo 1 } & \multicolumn{2}{c|}{ grupo 2 } & \multicolumn{2}{c|}{ grupo 3 } \\
\multicolumn{1}{c|}{} & $\hat{\beta}$ & $\hat{\gamma}$ & $\hat{\beta}$ & $\hat{\gamma}$ & $\hat{\beta}$ & $\hat{\gamma}$ \\
\hline$p_{\text {sus }}=1$ & 1.132 & 0.8847 & 1.177 & 0.8567 & 1.269 & 0.807 \\
$p_{\text {sus }}=0.8$ & 1.415 & 0.8839 & 1.473 & 0.8559 & 1.594 & 0.8073 \\
$p_{\text {sus }}=0.6$ & 1.89 & 0.8845 & 1.972 & 0.8565 & 2.142 & 0.8066 \\
$p_{\text {sus }}=0.4$ & 2.84 & 0.8841 & 2.982 & 0.8574 & 3.268 & 0.8071 \\
\hline
\end{tabular}

Tabela 4.7: Resumo dos estimadores encontrados para cada valor de $p_{\text {sus }}$

$$
\begin{aligned}
& a_{1}(\mathbf{X}(t), \beta)=\beta g_{1}(\mathbf{X}(t))=\beta \frac{S(t) I(t)}{N} \\
& a_{2}(\mathbf{X}(t), \gamma)=\gamma g_{2}(\mathbf{X}(t))=\gamma I(t)
\end{aligned}
$$

A redução de $p_{\text {sus }}$ implica na redução do número de indivíduos suscetíveis, e a consequente diminuição do valor de $g_{1}(\mathbf{X}(t))$. A queda de $g_{1}(\mathbf{X}(t))$ deve ser compensada pelo aumento do parâmetro $\beta$ com a finalidade de manter inalteradas os valores de $a_{1}(\mathbf{X}(t), \beta)$ e $a_{2}(\mathbf{X}(t), \gamma)$ uma vez que, por definição, essas funções regem as leis de transição de estados.

Por outro lado, observamos na tabela 4.7 que a estimativa de $\gamma$ permanece inalterada para diferentes valores de $p_{\text {sus }}$, conforme o esperado, já que a função $a_{2}(\mathbf{X}(t), \gamma)$ independe dos sintomáticos.

Observando a figura 4.16, notemos que à curto prazo, a diminuição do número de suscetíveis praticamente não afeta a dinâmica do sistema.

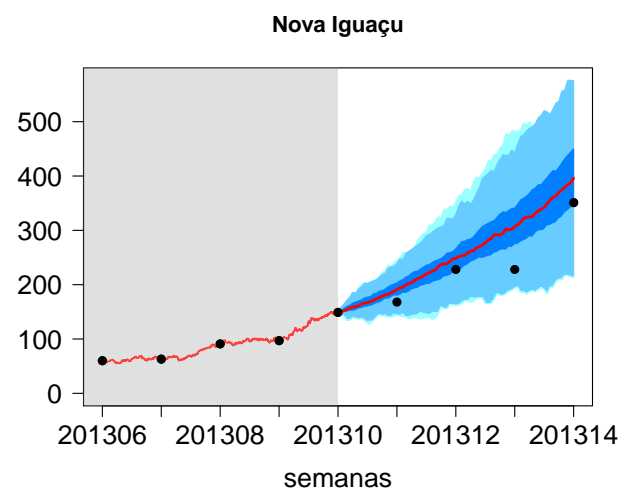

(a) Nova Iguaçu $\left(p_{\text {sus }}=1\right)$

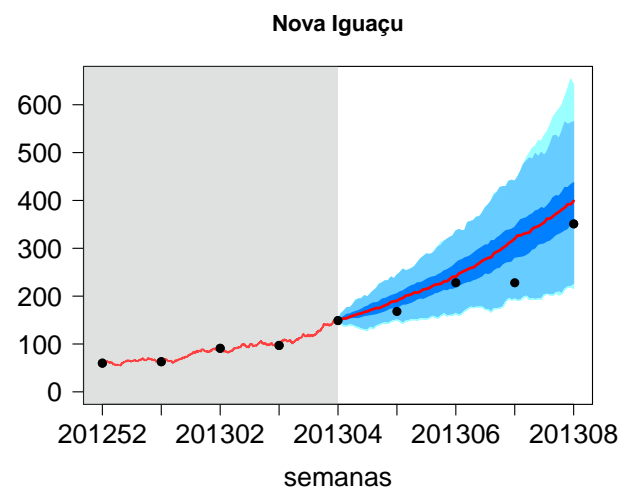

(c) Nova Iguaçu $\left(p_{\text {sus }}=0.6\right)$

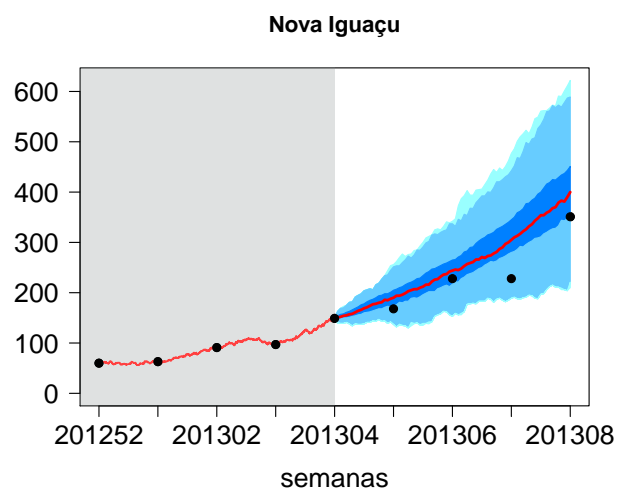

(b) Nova Iguaçu $\left(p_{\text {sus }}=0.8\right)$

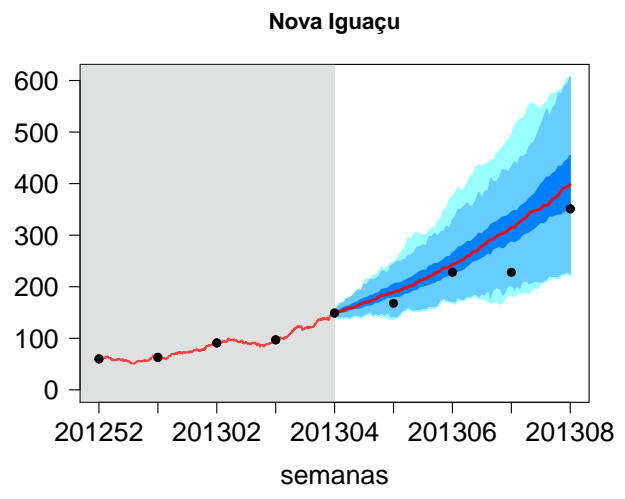

(d) Nova Iguaçu $\left(p_{\text {sus }}=0.4\right)$

Figura 4.16: Projeções geradas pela simulação do algoritmo de Gillespie para diferentes valores de $p_{\text {sus }}$ para a cidade de Nova Iguaçu para as semanas seguintes ao período de ajuste 
Vamos agora efetuar um outro teste, ampliando o período de previsão do modelo. Aqui, entretanto não temos a pretensão de que as curvas simuladas ajustam-se aos dados observados, desejamos discutir o efeito a longo prazo da variação do parâmetro $p_{\text {sus }}$ no sistema.

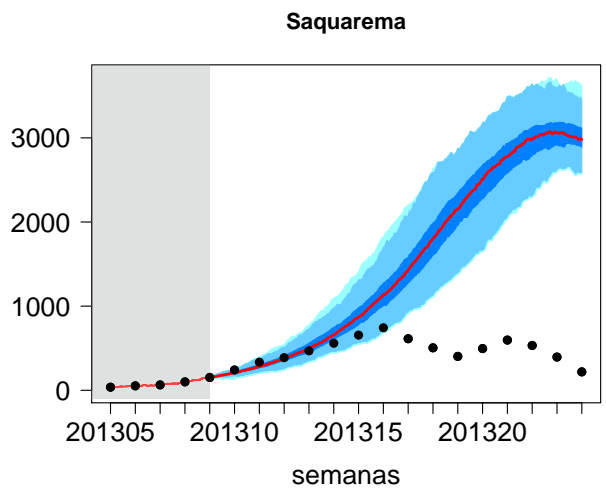

(a) Saquarema $\left(p_{\text {sus }}=1\right)$

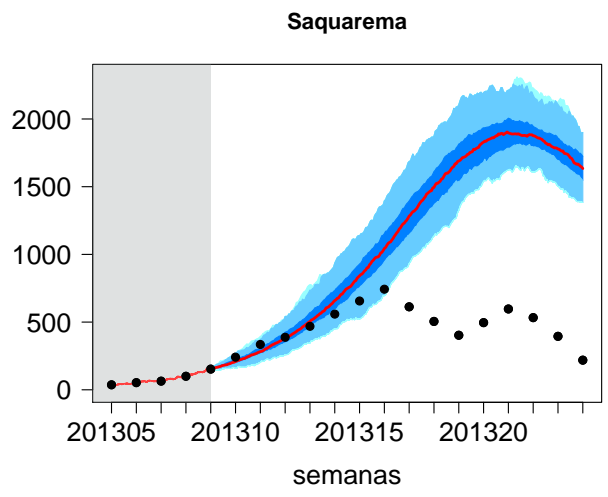

(c) Saquarema $\left(p_{\text {sus }}=0.6\right)$

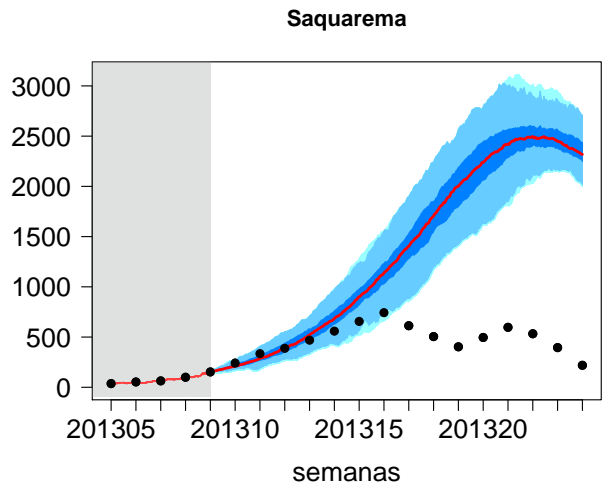

(b) Saquarema $\left(p_{\text {sus }}=0.8\right)$

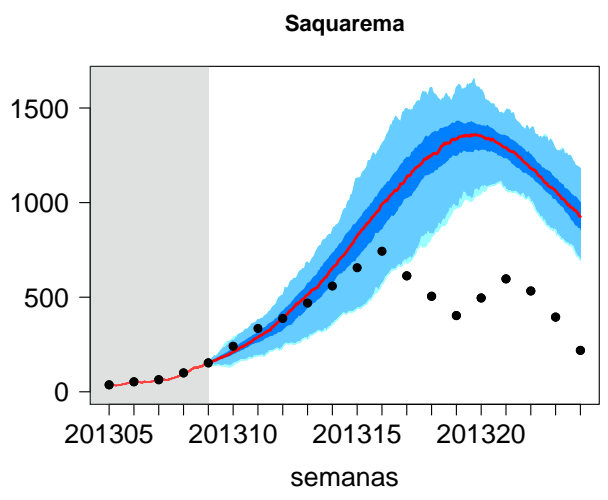

(d) Saquarema $\left(p_{\text {sus }}=0.4\right)$

Figura 4.17: Projeções geradas pela simulação do algoritmo de Gillespie para diferentes valores de $p_{\text {sus }}$ para a cidade de Saquarema com periodo de previsão estendido

Como podemos observar nas trajetórias obtidas para a cidade de Saquarema com período de previsão ampliado, detalhado na figura 4.17, que a longo prazo, conforme reduzimos o valor de $p_{\text {sus }}$ temos o efeito de antecipação do início da tendência descendente da curva de infectados. Esse comportamento é justificado pelo fato da diminuição de $p_{\text {sus }}$ implicar na menor oferta de indivíduos suscetíveis no sistema. 


\section{Capítulo 5}

\section{Aproximações por processos de difusão}

A simulação através do modelo de Gillespie exibe todos os eventos ocorridos no período de estudo: a cada reação deflagrada atualiza-se o estado do sistema e deve-se atualizar as funções de propensidade, que regem as leis para o próximo evento. Tal procedimento é computacionalmente oneroso, principalmente no caso em que muitos eventos ocorrem em um pequeno intervalo de tempo. Para processos com essas características, proporemos aqui uma alternativa. A notação empregada segue próxima a adotada por Dargatz [Dar07] e Fuchs [Fuc13].

Vamos denotar por $P_{N}(S, I ; t)$ a probabilidade do sistema com $N$ elementos apresentar $S$ indivíduos suscetíveis e $I$ infectados no instante $t$. Então, dado um intervalo de tempo de comprimento infinitesimal $\Delta t$, e lembrando que nosso sistema admite apenas dois canais de reação (infecção e recuperação), então para $(S, I) \in[0, N-1] \times[1, N-1] \operatorname{com} S+I \leq N$ temos:

$$
\begin{aligned}
P_{N}(S, I ; t+\Delta t) & =\beta \frac{(S+1)(I-1)}{N} \Delta t \times P_{N}(S+1, I-1 ; t)+ \\
& +\gamma(I+1) \Delta t \times P_{N}(S, I+1 ; t)+ \\
& +\left[1-\left(\beta \frac{S I}{N}+\gamma I\right) \Delta t\right] \times P_{N}(S, I ; t)
\end{aligned}
$$

Notemos que na expressão 5.1 tomamos $\Delta t$ tal que, no máximo, uma reação ocorre no intervalo $[t, t+\Delta t]$. Subtraindo $P(S, I, R ; t)$ de ambos os lados e dividindo por $\Delta t$, obtemos uma versão da Master Equation para nosso sistema:

$$
\begin{aligned}
\frac{\partial}{\partial t} P_{N}(S, I ; t) & =\beta \frac{(S+1)(I-1)}{N} P_{N}(S+1, I-1 ; t)+\gamma(I+1) P_{N}(S, I+1 ; t)+ \\
& -\left(\beta \frac{S I}{N}+\gamma I\right) P_{N}(S, I ; t)
\end{aligned}
$$

Além disso, temos as seguintes condições de fronteira:

$$
\begin{aligned}
\frac{\partial}{\partial t} P_{N}(S, 0 ; t) & =\gamma P_{N}(S, 1 ; t), \text { se } S<N \\
\frac{\partial}{\partial t} P_{N}(N, 0 ; t) & =0 \\
\frac{\partial}{\partial t} P_{N}(0, I ; t) & =\beta \frac{(I-1)}{N} P_{N}(1, N-1 ; t)-\gamma I P_{N}(0, I ; t)
\end{aligned}
$$

As equações de 5.2 a 5.5 são sujeitas à uma condição inicial $\left(S_{0}, I_{0}\right)$ no instante de tempo $t_{0}=0$.

Vamos agora exibir a transição do espaço de estados discreto para um sistema com espaço de estados contínuo, através da introdução das variáveis: 


$$
s=\frac{S}{N}, \quad i=\frac{I}{N}
$$

que representam as frações dos indivíduos suscetíveis, infectados e recuperados, respectivamente, na população total. É claro então que $s, i \in[0,1]$ e $s+i \leq 1$.

Vamos construir uma sequência de processos correspondendo a uma sequência de números $N$, que tende a infinito. Definimos agora as funções:

$$
\begin{aligned}
& \alpha_{1}(s, i)=\beta s i=\beta \frac{S I}{N^{2}} \\
& \alpha_{2}(s, i)=\gamma i=\gamma \frac{I}{N}
\end{aligned}
$$

Denotamos ainda por $p_{N}(s, i ; t)$ a probabilidade de um sistema com $N$ elementos possuir frações $s$ e $i$ de indivíduos suscetíveis e infectados, respectivamente, da população total no instante $t$, com condições iniciais fixadas. Notemos que: $p_{N}(s, i ; t)=P_{N}(N s, N i ; t)=P_{N}(S, I ; t)$.

Podemos então reescrever a master equation 5.2 para o processo intensivo:

$$
\begin{aligned}
\frac{\partial}{\partial t} p_{N}(s, i ; t) & =\frac{\alpha_{1}(s+\epsilon, i-\epsilon)}{\epsilon} p_{N}(s+\epsilon, i-\epsilon ; t)+\frac{\alpha_{2}(s, i+\epsilon)}{\epsilon} p_{N}(s, i+\epsilon ; t)+ \\
& -\frac{\left[\alpha_{1}(s, i)+\alpha_{2}(s, i)\right]}{\epsilon} p_{N}(s, i ; t) \\
& =\frac{\alpha_{1}(s+\epsilon, i-\epsilon)}{\epsilon} p_{N}(s+\epsilon, i-\epsilon ; t)-\frac{\alpha_{1}(s, i)}{\epsilon} p_{N}(s, i ; t)+ \\
& +\frac{\alpha_{2}(s, i+\epsilon)}{\epsilon} p_{N}(s, i+\epsilon ; t)-\frac{\alpha_{2}(s, i)}{\epsilon} p_{N}(s, i ; t)
\end{aligned}
$$

onde $\epsilon=1 / N$.

Definindo agora por $p$ o limite das sequências $p_{N}$, temos:

$$
\begin{aligned}
\frac{\partial}{\partial t} p(s, i ; t) & =\frac{\alpha_{1}(s+\epsilon, i-\epsilon) p(s+\epsilon, i-\epsilon ; t)-\alpha_{1}(s, i) p(s, i ; t)}{\epsilon}+ \\
& +\frac{\alpha_{2}(s, i+\epsilon) p(s, i+\epsilon ; t)-\alpha_{2}(s, i) p(s, i ; t)}{\epsilon}
\end{aligned}
$$

Adicionando e subtraindo alguns termos do lado direito de 5.7, conforme sugerido por Dargatz [Dar07], obtemos:

$$
\begin{aligned}
& \frac{1}{\epsilon}\left[\alpha_{1}(s+\epsilon, i-\epsilon) p(s+\epsilon, i-\epsilon ; t)+\alpha_{1}(s+\epsilon, i) p(s+\epsilon, i ; t)-\alpha_{1}(s+\epsilon, i) p(s+\epsilon, i ; t)+\right. \\
& +\frac{\alpha_{1}(s-\epsilon, i) p(s-\epsilon, i ; t)}{2}-\frac{\alpha_{1}(s-\epsilon, i) p(s-\epsilon, i ; t)}{2}+\frac{\alpha_{1}(s, i+\epsilon) p(s, i+\epsilon ; t)}{2}-\frac{\alpha_{1}(s, i+\epsilon) p(s, i+\epsilon ; t)}{2}+ \\
& +\alpha_{1}(s, i-\epsilon) p(s, i-\epsilon ; t)-\alpha_{1}(s, i-\epsilon) p(s, i-\epsilon ; t)+2 \alpha_{1}(s, i) p(s, i ; t)-3 \alpha_{1}(s, i) p(s, i ; t)+ \\
& +\alpha_{2}(s, i+\epsilon) p(s, i+\epsilon ; t)+\frac{\alpha_{2}(s, i-\epsilon) p(s, i-\epsilon ; t)}{2}-\frac{\alpha_{2}(s, i-\epsilon) p(s, i-\epsilon ; t)}{2}+ \\
& \left.+\frac{\alpha_{2}(s, i) p(s, i ; t)}{2}-\frac{3 \alpha_{2}(s, i) p(s, i ; t)}{2}\right]
\end{aligned}
$$

A justificativa dos termos inseridos na expressão 5.8 podem ser encontrados no trabalho de Fuchs [Fuc13], onde é apresentado inclusive um algoritmo para determinar os termos incorporados. 
Detalhes também podem ser encontrados no trabalho de Bulhosa [Bul16].

Rearranjando os termos de 5.8 de forma conveniente:

$$
\begin{aligned}
& \frac{1}{2 \epsilon}\left[\alpha_{1}(s+\epsilon, i) p(s+\epsilon, i ; t)-\alpha_{1}(s, i) p(s, i ; t)\right]+\frac{1}{2 \epsilon}\left[\alpha_{1}(s, i) p(s, i ; t)-\alpha_{1}(s-\epsilon, i) p(s-\epsilon, i ; t)\right]+ \\
- & \frac{1}{2 \epsilon}\left[\alpha_{1}(s, i+\epsilon) p(s, i+\epsilon ; t)-\alpha_{2}(s, i+\epsilon) p(s, i+\epsilon ; t)-\alpha_{1}(s, i) p(s, i ; t)+\alpha_{2}(s, i) p(s, i ; t)\right]+ \\
- & \frac{1}{2 \epsilon}\left[\alpha_{1}(s, i) p(s, i ; t)-\alpha_{2}(s, i) p(s, i ; t)-\alpha_{1}(s, i-\epsilon) p(s, i-\epsilon ; t)+\alpha_{2}(s, i-\epsilon) p(s, i-\epsilon ; t)\right]+ \\
+ & \frac{1}{2 \epsilon}\left[\alpha_{1}(s+\epsilon, i) p(s+\epsilon, i ; t)-2 \alpha_{1}(s, i) p(s, i ; t)+\alpha_{1}(s-\epsilon, i) p(s-\epsilon, i ; t)\right]+ \\
+ & \frac{1}{2 \epsilon}\left[\alpha_{1}(s, i+\epsilon) p(s, i+\epsilon ; t)+\alpha_{2}(s, i+\epsilon) p(s, i+\epsilon ; t)-2 \alpha_{1}(s, i) p(s, i ; t)-2 \alpha_{2}(s, i) p(s, i ; t)+\right. \\
& \left.+\alpha_{1}(s, i-\epsilon) p(s, i-\epsilon ; t)+\alpha_{2}(s, i-\epsilon) p(s, i-\epsilon ; t)\right]+ \\
- & \frac{1}{\epsilon}\left[\alpha_{1}(s+\epsilon, i) p(s+\epsilon, i ; t)-\alpha_{1}(s+\epsilon, i-\epsilon) p(s+\epsilon, i-\epsilon ; t)-\alpha_{1}(s, i) p(s, i ; t)+\alpha(s, i-\epsilon) p(s, i-\epsilon ; t)\right]
\end{aligned}
$$

Analisando agora os termos da equação 5.9, tomando o limite $\epsilon \rightarrow 0$. Da primeira linha, temos:

$$
\begin{aligned}
& \frac{1}{2 \epsilon}\left[\alpha_{1}(s+\epsilon, i) p(s+\epsilon, i ; t)-\alpha_{1}(s, i) p(s, i ; t)+\alpha_{1}(s, i) p(s, i ; t)-\alpha_{1}(s-\epsilon, i) p(s-\epsilon, i ; t)\right]= \\
& =\frac{1}{2}\left[\frac{\beta(s+\epsilon) i p(s+\epsilon, i ; t)-\beta s i p(s, i ; t)}{\epsilon}+\frac{\beta s i p(s, i ; t)-\beta(s-\epsilon) i p(s-\epsilon, i ; t)}{\epsilon}\right] \\
& \rightarrow \frac{\partial}{\partial s} \beta s i p(s, i ; t)
\end{aligned}
$$

Usando as expressões da segunda e terceira linhas:

$$
\begin{aligned}
& \frac{1}{2}\left[\frac{\alpha_{1}(s, i+\epsilon) p(s, i+\epsilon ; t)-\alpha_{1}(s, i) p(s, i ; t)}{\epsilon}-\frac{\alpha_{2}(s, i+\epsilon) p(s, i+\epsilon ; t)-\alpha_{2}(s, i) p(s, i ; t)}{\epsilon}+\right. \\
& +\frac{1}{2 \epsilon}\left[\frac{\alpha_{1}(s, i) p(s, i ; t)-\alpha_{1}(s, i-\epsilon) p(s, i-\epsilon ; t)}{\epsilon}-\frac{\alpha_{2}(s, i) p(s, i ; t)-\alpha_{2}(s, i-\epsilon) p(s, i-\epsilon ; t)}{\epsilon}\right] \\
& =\frac{1}{2}\left\{\frac{[\beta s i-\gamma i] p(s, i ; t)-[\beta s(i-\epsilon)-\gamma(i-\epsilon)] p(s, i-\epsilon ; t)}{\epsilon}\right\} \\
& \rightarrow \frac{\partial}{\partial i}(\beta s i-\gamma i) p(s, i ; t)
\end{aligned}
$$

Da quarta linha de 5.9:

$$
\begin{aligned}
& \frac{1}{2 \epsilon}\left[\alpha_{1}(s+\epsilon, i) p(s+\epsilon, i ; t)-2 \alpha_{1}(s, i) p(s, i ; t)+\alpha_{1}(s-\epsilon, i) p(s-\epsilon, i ; t)\right]+ \\
& =\frac{1}{2}\left[\frac{\beta(s+\epsilon) i p(s+\epsilon, i ; t)-2 \beta s i p(s, i ; t)+\beta(s-\epsilon) i p(s-\epsilon, i ; t)}{\epsilon}\right] \\
& \rightarrow \frac{\partial^{2}}{\partial s^{2}} \frac{1}{N} \beta s i p(s, i ; t)
\end{aligned}
$$

O próximo termo a ser analisado nos fornece: 


$$
\begin{aligned}
& \frac{1}{2}\left[\frac{\left(\alpha_{1}(s, i+\epsilon)+\alpha_{2}(s, i+\epsilon)\right) p(s, i+\epsilon ; t)}{\epsilon}-2 \frac{\left(\alpha_{1}(s, i)+\alpha_{2}(s, i)\right) p(s, i ; t)}{\epsilon}+\right. \\
& \left.+\frac{\left(\alpha_{1}(s, i-\epsilon)+\alpha_{2}(s, i-\epsilon)\right) p(s, i-\epsilon ; t)}{\epsilon}\right] \\
& =\frac{1}{2}\left[\frac{(\beta s(i+\epsilon)+\gamma(i+\epsilon)) p(s, i+\epsilon ; t)-2(\beta s i+\gamma i) p(s, i ; t)+(\beta s(i-\epsilon)+\gamma(i-\epsilon)) p(s, i-\epsilon ; t)}{\epsilon}\right] \\
& \rightarrow \frac{1}{2} \frac{\partial^{2}}{\partial i^{2}} \frac{1}{N}[\beta s i-\gamma i] p(s, i ; t)
\end{aligned}
$$

Finalmente, da última linha de 5.9:

$$
\begin{aligned}
& {\left[\frac{\alpha_{1}(s+\epsilon, i) p(s+\epsilon, i ; t)-\alpha_{1}(s+\epsilon, i-\epsilon) p(s+\epsilon, i-\epsilon ; t)-\alpha_{1}(s, i) p(s, i ; t)+\alpha(s, i-\epsilon) p(s, i-\epsilon ; t)}{\epsilon}\right]} \\
& =\left[\frac{\beta(s+\epsilon) i p(s+\epsilon, i ; t)-\beta(s+\epsilon)(i-\epsilon) p(s+\epsilon, i-\epsilon ; t)-\beta s i p(s, i ; t)+\beta s(i-\epsilon) p(s, i-\epsilon ; t)}{\epsilon}\right] \\
& \rightarrow \frac{\partial^{2}}{\partial s \partial i} \frac{1}{N} \beta s i p(s, i ; t)
\end{aligned}
$$

Reunindo agora as informações obtidas através das expressões entre 5.7 e 5.14, obtemos:

$$
\begin{aligned}
\frac{\partial}{\partial t} p(s, i ; t) & =\frac{\partial}{\partial s} \beta s i p(s, i ; t)-\frac{\partial}{\partial i}(\beta s i-\gamma i) p(s, i ; t)+\frac{\partial^{2}}{\partial s^{2}} \frac{1}{N} \beta s i p(s, i ; t)+ \\
& +\frac{1}{2} \frac{\partial^{2}}{\partial i^{2}} \frac{1}{N}[\beta s i-\gamma i] p(s, i ; t)-\frac{\partial^{2}}{\partial s \partial i} \frac{1}{N} \beta s i p(s, i ; t)
\end{aligned}
$$

Essa expressão pode ser escrita como:

$$
\frac{\partial}{\partial t} p(\boldsymbol{x} ; t)=-\sum_{j=1}^{2} \frac{\partial}{\partial x^{j}} \mu_{j}(\boldsymbol{x}) p(\boldsymbol{x} ; t)+\frac{1}{2 N} \sum_{j, k=1}^{2} \frac{\partial^{2}}{\partial x^{j} \partial x^{k}} \Sigma_{j k}(\boldsymbol{x}) p(\boldsymbol{x}, t)
$$

onde $\boldsymbol{x}=\left(x^{1}, x^{2}\right)^{\prime}=(s, i)^{\prime}{ }^{1} \mathrm{e}$

$$
\begin{aligned}
\boldsymbol{\mu}(\boldsymbol{x}) & =\left(\begin{array}{l}
\mu_{1}(\boldsymbol{x}) \\
\mu_{2}(\boldsymbol{x})
\end{array}\right)=\left(\begin{array}{c}
-\alpha_{1}(\boldsymbol{x}) \\
\alpha_{1}(\boldsymbol{x})-\alpha_{2}(\boldsymbol{x})
\end{array}\right)=\left(\begin{array}{c}
-\beta s i \\
\beta s i-\gamma i
\end{array}\right) \\
\boldsymbol{\Sigma}(\boldsymbol{x}) & =\left(\begin{array}{ll}
\Sigma_{11}(\boldsymbol{x}) & \Sigma_{12}(\boldsymbol{x}) \\
\Sigma_{21}(\boldsymbol{x}) & \Sigma_{22}(\boldsymbol{x})
\end{array}\right)=\left(\begin{array}{cc}
\alpha_{1}(\boldsymbol{x}) & -\alpha_{1}(\boldsymbol{x}) \\
-\alpha_{1}(\boldsymbol{x}) & \alpha_{1}(\boldsymbol{x})+\alpha_{2}(\boldsymbol{x})
\end{array}\right)=\left(\begin{array}{cc}
\beta s i & -\beta s i \\
-\beta s i & \beta s i+\gamma i
\end{array}\right)
\end{aligned}
$$

A equação 5.15 é conhecida como Equação de Fokker-Planck ou Equação de difusão progressiva de Kolmogorov (forward Kolmogorov diffusion equation). $\boldsymbol{\mu}$ é chamado de termo flutuante (drift term) e $\boldsymbol{\Sigma} / N$ é chamado de termo difusivo (diffusion term) [Dar07].

Tomando $s$ e $i$ positivos temos que o termo difusivo $\boldsymbol{\Sigma} / N$ é definido positivo. Isso implica que o processo Markoviano com espaço de estados e tempo contínuos pode ser aproximado pela difusão, solução da equação diferencial estocástica (Stochastic Differential Equation - SDE) [Fuc13]:

$$
\left\{\begin{array}{l}
\boldsymbol{x}_{t_{0}}=\boldsymbol{x}_{0} \\
d \boldsymbol{x}_{t}=\boldsymbol{\mu}\left(\boldsymbol{x}_{t}\right) d t+\frac{1}{\sqrt{N}} \boldsymbol{\sigma}\left(\boldsymbol{x}_{t}\right) d \mathbf{B}_{t}
\end{array}\right.
$$

\footnotetext{
${ }^{1}$ Não confundir com o vetor de estados do contínuo $\boldsymbol{x}$ com o vetor do modelo discreto $\mathbf{x}$
} 
onde $\boldsymbol{\sigma}$ é tal que: $\boldsymbol{\Sigma}=\boldsymbol{\sigma} \boldsymbol{\sigma}^{\prime}$. Conforme observado por Fuchs [Fuc13], a decomposição $\boldsymbol{\Sigma}=\boldsymbol{\sigma} \boldsymbol{\sigma}^{\prime}$ não é única, tomaremos $\sigma$ na forma:

$$
\boldsymbol{\sigma}=\left(\begin{array}{cc}
\sqrt{\beta s i} & 0 \\
-\sqrt{\beta s i} & \sqrt{\gamma i}
\end{array}\right)
$$

Recapitulando, dados um sistema do tipo SIR com N indivíduos, e um processo estocástico de contagem associado, cujo espaço de estados exibe o número de indivíduos suscetíveis $(S)$ e infectados (I) para todo instante de tempo $t$, temos a equação mestra associada dada por 5.2. Através da introdução de variáveis intensivas $s=S / N$ e $i=I / N$, podemos construir um processo contínuo associado, cuja equação mestra é apresentada na expressão 5.6.

Definindo $p$ como o limite da sequência $p_{N}$, obtemos:

$$
\begin{aligned}
\frac{\partial}{\partial t} p(s, i ; t) & =\frac{\alpha_{1}(s+\epsilon, i-\epsilon) p(s+\epsilon, i-\epsilon ; t)-\alpha_{1}(s, i) p(s, i ; t)}{\epsilon}+ \\
& +\frac{\alpha_{2}(s, i+\epsilon) p(s, i+\epsilon ; t)-\alpha_{2}(s, i) p(s, i ; t)}{\epsilon}
\end{aligned}
$$

que, após a soma e subtração de termos de forma conveniente, pode ser escrita na forma apresentada em 5.15 .

Esse processo, por fim, pode ser aproximado por um processo difusivo, solução da SDE:

$$
\left\{\begin{array}{l}
d s=-\beta s i d t+\sqrt{\frac{\beta s i}{N}} d B_{1} \\
d i=(\beta s i-\gamma i) d t-\sqrt{\frac{\beta s i}{N}} d B_{1}+\sqrt{\frac{\gamma i}{N}} d B_{2}
\end{array}\right.
$$

com uma condição inicial $\left(s_{0}, i_{0}\right)^{\prime}$ apropriada.

$B_{1}$ e $B_{2}$ em 5.18 são movimentos Brownianos independentes.

Notemos agora que, tomando o limite $N \rightarrow+\infty$, temos:

$$
\left\{\begin{array}{l}
d s=-\beta s i d t \\
d i=(\beta s i-\gamma i) d t
\end{array}\right.
$$

que é o sistema de equações diferenciais ordinárias que descreve a dinâmica do modelo de forma determinística.

$\mathrm{s}$

\subsection{Uma outra abordagem}

Abordaremos agora o problema através de aproximações do algoritmo de Gillespie. A notação adotada segue próxima a adotada por Higham [Hig08] e Gillespie [Gil08].

\subsubsection{Tau-leaping}

O método conhecido como tau-leaping é recomendado para sistema em que o intervalo de tempo de espera entre as reações é pequeno. Dados o estado do sistema $\mathbf{X}(t)=\mathbf{x}$ em um instante $t$ e $\tau>0$ fixado, o método calcula o estado $\mathbf{X}(t+\tau)$ atualizando o sistema com todas as reações esperadas para o intervalo $[t, t+\tau]$, utilizando relações dadas pelas funções de propensidade calculadas no instante $t$. Em outras palavras, para cada reação $\mathcal{R}_{k}$, utilizamos sua respectiva função de propensidade 
$a_{k}\left(\mathbf{X}(t), \theta_{k}\right)$ no instante $t$, para determinar o número de reações esperadas no intervalo $[t, t+\tau]$. Os números de eventos obtidos são usados para atualizar o estado do sistema:

$$
\mathbf{X}(t+\tau)=\mathbf{X}(t)+\sum_{j=1}^{M} \nu_{j} \mathcal{P}_{j}\left(a_{j}\left(\mathbf{X}(t), \theta_{j}\right) \tau\right)
$$

onde $M$ é o número de canais reativos admitidos no sistema (no nosso caso dois: infecção e recuperação) e $\mathcal{P}_{j}\left(a_{j}\left(\mathbf{X}(t), \theta_{j}\right), \tau\right)$ são variáveis aleatórias cuja natureza será discutida adiante.

Notemos que o comprimento do intervalo $\tau$ não pode ser muito grande, para não distorcer o comportamento observado no sistema através do modelo de Gillespie. Ou seja, estamos interessados em agrupar um conjunto relativamente pequeno de reações em cada intervalo, de modo que as funções de propensidade $\left\{a_{k}\left(\mathbf{X}(t), \theta_{k}\right)\right\}_{k=1,2}$ não variem de forma significativa.

Da definição de função de propensidade, segue que a probabilidade de uma reação $k$ ocorrer em um intervalo de tempo $\Delta t$ é dado por $a_{k}\left(\mathbf{X}(t), \theta_{k}\right) \Delta t$. Então, ao manter as funções de propensidade $\left\{a_{k}\left(\mathbf{X}(t), \theta_{k}\right)\right\}_{k=1,2}$ inalteradas ao longo do intervalo $[t, t+\tau]$ nosso problema resume-se à análise de processos de contagem. Mais especificamente, temos processos de Poisson homogêneos com taxas dadas pelas funções de propensidade. Logo, se $\mathcal{P}_{j}\left(a_{j}\left(\mathbf{X}(t), \theta_{j}\right) \tau\right)$ é uma variável aleatória representando o número de eventos do tipo $\mathcal{R}_{j}$ em um intervalo $[t, t+\tau]$, então:

$$
\mathcal{P}_{j}\left(a_{j}\left(\mathbf{X}(t), \theta_{j}\right) \tau\right) \sim \operatorname{Poisson}\left(a_{j}\left(\mathbf{X}(t), \theta_{j}\right) \tau\right)
$$

Detalhes sobre processos de Poisson podem ser encontrados na seção A.1.

O algoritmo 6 apresenta a metodologia do Tau-Leaping Method.

Algoritmo 6 Tau-Leaping

ENTRADA: instante inicial $t_{0}$; instante final $t_{f}$; estado inicial $\mathbf{x}_{0}$; parâmetros do modelo $\boldsymbol{\theta}=$ $\left(\theta_{1}, \theta_{2}\right)$; funções de propensidade $a_{k}\left(\mathbf{x}, \theta_{k}\right)$; vetores de mudança de estados $\nu_{k}, k=1,2$; tamanho do passo temporal $\tau$

SAÍDA: sequência de duplas $\left(t_{j}, \mathbf{x}_{j}\right)$ que descreve uma trajetória do sistema

1: função TAU-LEAPing $\left(t_{0}, t_{f}, \mathbf{x}_{0}, \tau\right)$

2: $\quad i \leftarrow 1$

3: $\quad T \leftarrow t_{0}, t[i] \leftarrow T, \mathbf{x}[i] \leftarrow \mathbf{x}_{0}$

4: $\quad$ enquanto $T \leq t_{f}$ faça

5: $\quad$ calcule $a_{k}\left(\mathbf{x}[i], \theta_{k}\right)$ para $k=1,2$

$\triangleright$ Cálculo da função de propensidade

gere $p_{k} \sim \operatorname{Poisson}\left(a_{k}\left(\mathbf{x}[i], \theta_{k}\right) \tau\right)$ para $k=1,2$

$\mathbf{x}[i+1] \leftarrow \mathbf{x}[i]+p_{1} \nu_{1}+p_{2} \nu_{2} \quad \triangleright$ Atualizando todas as reações do intervalo

$t[i+1] \leftarrow T+\tau$

$T \leftarrow T+\tau$

$i \leftarrow i+1$

\section{fim enquanto}

devolve $(t, \mathbf{x})$

fim função

$\triangleright$ sequências dos tempos de ocorrência e dos estados do sistema

\subsubsection{A aproximação por processos de difusão}

O método de tau-leaping nos fornece uma aproximação do método de Gillespie em que o número de eventos ocorridos de um determinado canal reativo em um intervalo dado é uma variável aleatória com distribuição Poisson.

Vamos relembrar a seguinte propriedade da distribuição Poisson: 


$$
X \sim \operatorname{Poisson}(\lambda) \Rightarrow\left\{\begin{array}{l}
E(X)=\lambda \\
\operatorname{Var}(X)=\lambda
\end{array}\right.
$$

Um outro resultado importante é apresentado a seguir:

Proposição 5.1.1. Seja $X \sim$ Poisson $(\lambda)$. Se $\lambda \gg 1$ então temos aproximadamente $X \approx$ Normal $(\lambda, \lambda)$, onde Normal $(a, b)$ representa a distribuição normal com média a e variância b [Gil08].

Utilizamos aqui o mesmo procedimento de fixar o valor das funções de propensidade ao longo de um intervalo $[t, t+\tau]$ e notamos que, nesse caso, o número de eventos de cada tipo $\mathcal{R}_{j}$ é uma variável aleatória com distribuição de Poisson $\mathcal{P}_{j}\left(a_{j}\left(\mathbf{X}(t), \theta_{j}\right), \tau\right)$, conforme discutido na seção 5.1.1.

Notemos que $\tau$ deve ser escolhido forma que as expressões $\lambda_{k}=a_{k}\left(\mathbf{X}(t), \theta_{k}\right) \tau$ para $k=1,2$ sejam suficientemente grandes que justifiquem a aproximação de distribuições de Poisson com taxas $\lambda_{k}$ por distribuições normais tal como proposto na proposição 5.1.1.

Outro ponto de atenção é o fato de que na proposição 5.1.1 aproximamos variáveis aleatórias discretas (Poisson) por variáveis aleatórias contínuas (Normal). É interessante então, assim como efetuado no início desse capítulo, trabalharmos com o processo intensivo. Vamos, de forma análoga a , definir $\boldsymbol{x}^{*}=(s, i, r)^{\prime}$, onde $s$ e $i$ representam a proporção de suscetíveis, infectados e recuperados em uma população de tamanho $N$.

Dessa forma, tomando $\epsilon=1 / N$, podemos reescrever a expressão 5.20:

$$
\boldsymbol{x}^{*}(t+\tau)=\boldsymbol{x}^{*}(t)+\epsilon \sum_{j=1}^{2} \nu_{j} \mathcal{P}_{j}\left(a_{j}\left(N \boldsymbol{x}^{*}(t), \theta_{j}\right) \tau\right)
$$

Notamos que agora, o vetor estequiométrico, que efetua a mudança de estados sofre uma reescala através do tamanho de passo $\epsilon$.

Utilizando a proposição 5.1.1, temos da expressão 5.21 que:

$$
\boldsymbol{x}^{*}(t+\tau)=\boldsymbol{x}^{*}(t)+\epsilon \sum_{j=1}^{2} \mathcal{N}_{j} \nu_{j}, \quad \text { onde } \mathcal{N}_{j} \sim \operatorname{Normal}\left(a_{j}\left(N \boldsymbol{x}^{*}(t), \theta_{j}\right) \tau, a_{j}\left(N \boldsymbol{x}^{*}(t), \theta_{j}\right) \tau\right)
$$

Sejam agora $Z_{j} \sim \operatorname{Normal}(0,1)$. Podemos reescrever a equação 5.22 na seguinte forma:

$$
\boldsymbol{x}^{*}(t+\tau)=\boldsymbol{x}^{*}(t)+\tau \epsilon \sum_{j=1}^{2} \nu_{j} a_{j}\left(N \boldsymbol{x}^{*}(t), \theta_{j}\right)+\sqrt{\tau} \epsilon \sum_{j=1}^{2} \nu_{j} \sqrt{a_{j}\left(N \boldsymbol{x}^{*}(t), \theta_{j}\right)} Z_{j}
$$

Denotando $\boldsymbol{x}^{*}(t)=(s(t), i(t), r(t))^{\prime}$ e lembrando das expressões das funções de propensidade e dos vetores estequiométricos, podemos reescrever 5.23:

$$
\left\{\begin{array}{l}
s(t+\tau)=s(t)-\tau \beta s(t) i(t)-\sqrt{\tau} \sqrt{\frac{\beta s(t) i(t)}{N}} Z_{1} \\
i(t+\tau)=i(t)+\tau[\beta s(t) i(t)-\gamma i(t)]+\sqrt{\tau} \sqrt{\frac{\beta s(t) i(t)}{N}} Z_{1}-\sqrt{\tau} \sqrt{\frac{\gamma i(t)}{N}} Z_{2} \\
r(t+\tau)=r(t)+\tau \gamma i(t)+\sqrt{\tau} \sqrt{\frac{\gamma i(t)}{N}} Z_{2}
\end{array}\right.
$$

onde $Z_{1}, Z_{2} \sim \operatorname{Normal}(0,1)$

Dessa forma, uma trajetória do processo pode ser simulada através do procedimento descrito no algoritmo 7. 


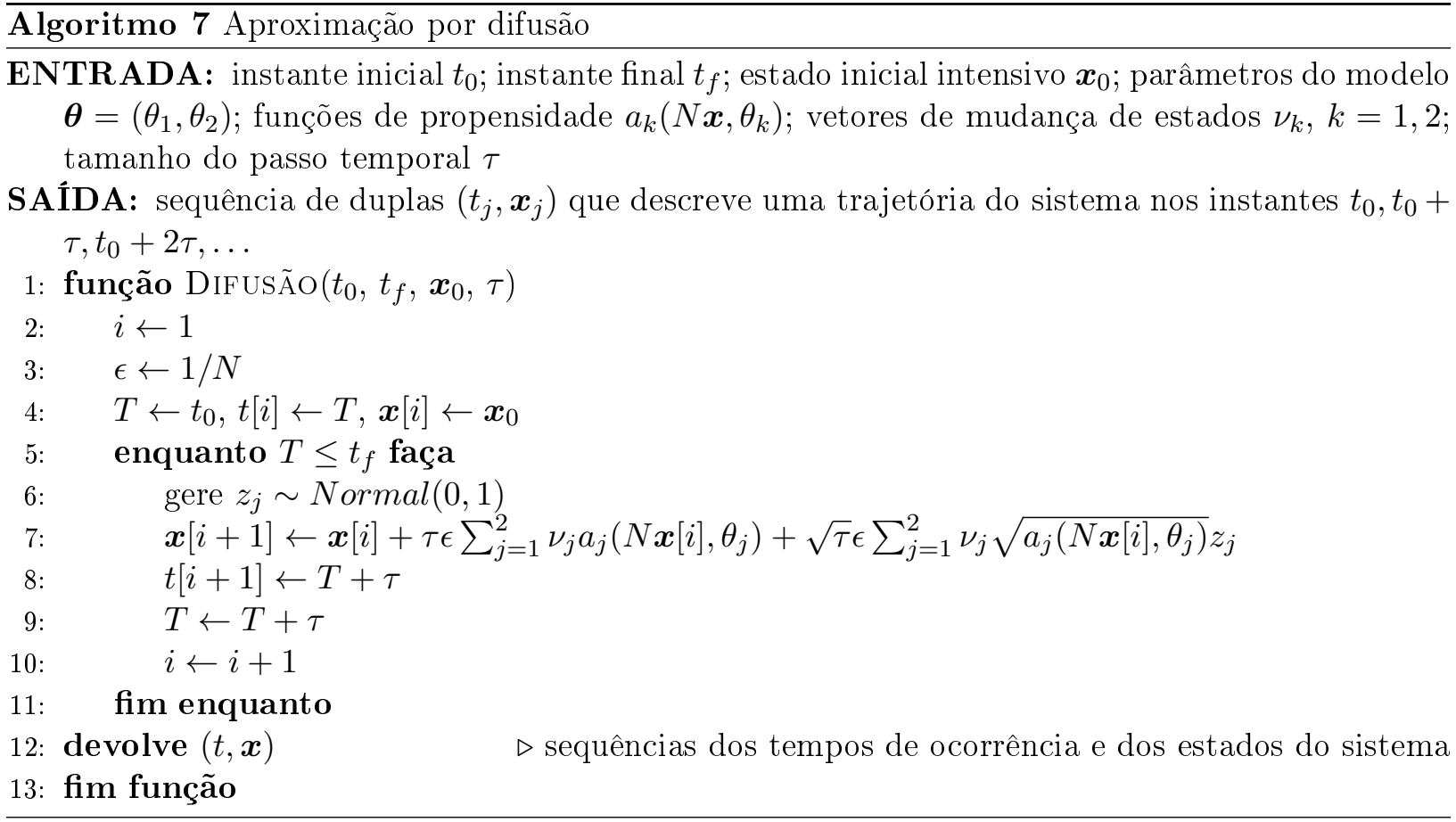

Seja agora $\boldsymbol{x}(t)$ o processo intensivo restrito às duas primeiras coordenadas de $\boldsymbol{x}^{*}$, ou seja, $\boldsymbol{x}(t)=(s(t), i(t))^{\prime}$, onde $s(t)$ e $i(t)$ podem ser calculadas para cada instante $t$ conforme explicitado na expressão 5.24.

Notemos que o procedimento adotado em 5.24 consiste exatamente na discretização do método de Euler-Maruyama, apresentado na seção B, para o problema em tempo contínuo apresentado em 5.18. Então, através da simulação do algoritmo 7, obtemos a aproximação do nosso modelo epidemiológico através de um processo difusivo.

Uma ideia simples da aplicação do algoritmo 7 é através do uso dos parâmetros estimados para o modelo discreto, cuja metodologia foi discutida em 3.

Apresentamos na figura 5.1 simulações de aproximações obtidas pelo modelo difusivo para o período pós ajuste na cidade do Rio de Janeiro com diferentes passos temporais $\tau$.

Observamos que as simulações obtidas capturam fielmente o comportamento observado no modelo de Gillespie (figura 5.1f). Para a cidade do Rio de Janeiro esse fenômeno é até, de certa forma, esperado uma vez que o número de infectados na fase inicial da doença é elevado, o que diminui a sensibilidade das funções de propensidade.

Analisamos também o caso de uma cidade com menor população e menor densidade demográfica. A cidade escolhida foi Teresópolis, que apresentou uma grande variabilidade nos cenários obtidos através da simulação do modelo de Gillespie, como observado nas figuras 4.10j e 5.2f. As aproximações fornecidas pelo modelo difusivo são apresentadas na figura 5.2.

Notemos que, mesmo nesse caso, as simulações obtidas pelos diferentes tamanhos de passo temporal capturam de forma surpreendente a tendência observada pelo modelo de Gillespie.

Testes foram realizados com as demais cidades e os resultados obtidos foram semelhantes.

Temos então que o modelo difusivo fornece uma alternativa satisfatória e menos dispendiosa computacionalmente para a obtenção de projeções de cenários para as semanas subsequentes ao período de ajuste.

Ao observarmos os gráficos obtidos nas figuras 5.1 e 5.2 podemos concluir que as funções de propensidade apresentam uma pequena variabilidade durante o período de previsão (semanas seguntes ao período de ajuste). O crescimento do número de infectados registrado no período de ajuste diminui a sensibilidade das funções de propensidade, o que torna possível uma aproximação acurada através do modelo difusivo. 


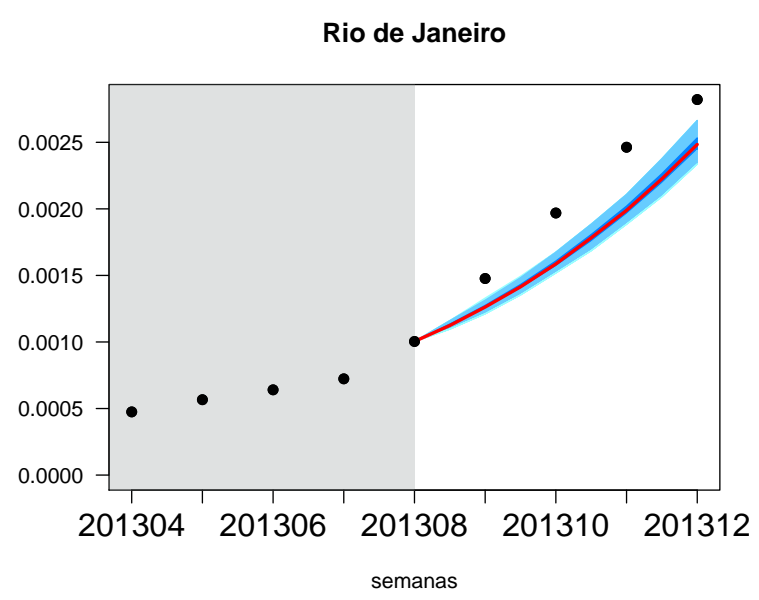

(a) $\tau=0.5$

\section{Rio de Janeiro}

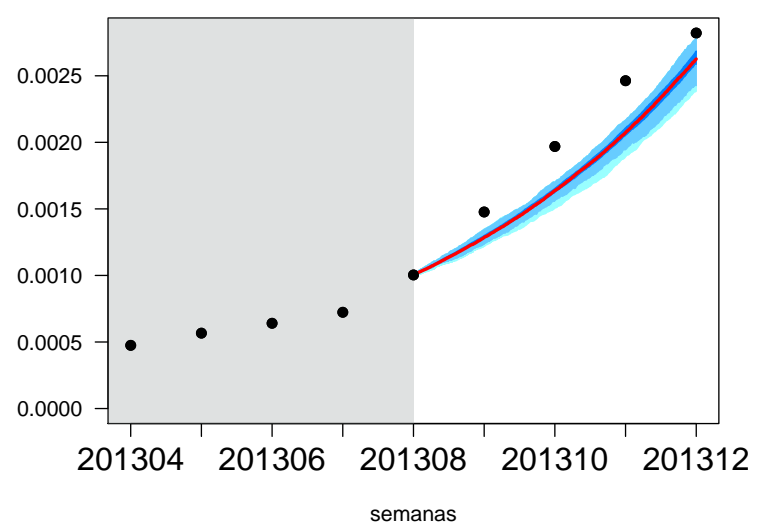

(c) $\tau=0.01$

Rio de Janeiro

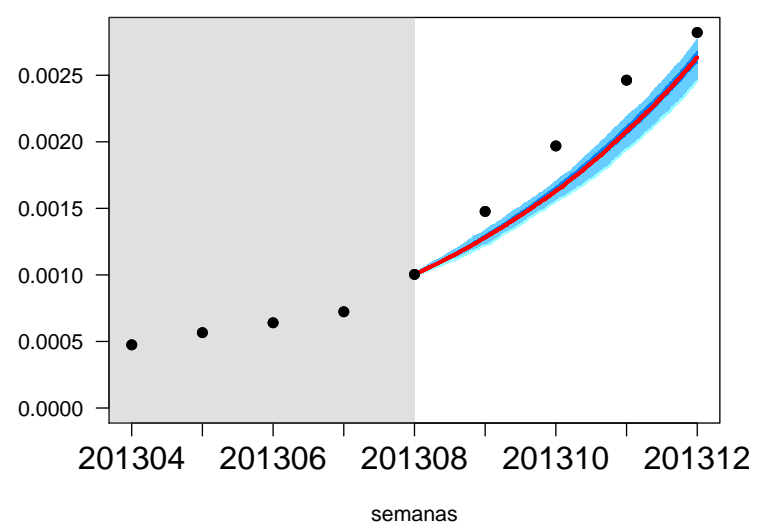

(e) $\tau=0.0001$

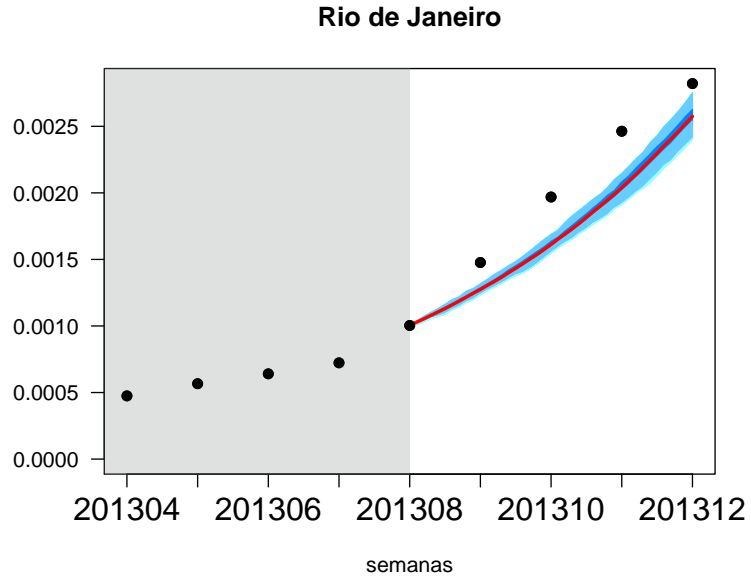

(b) $\tau=0.1$

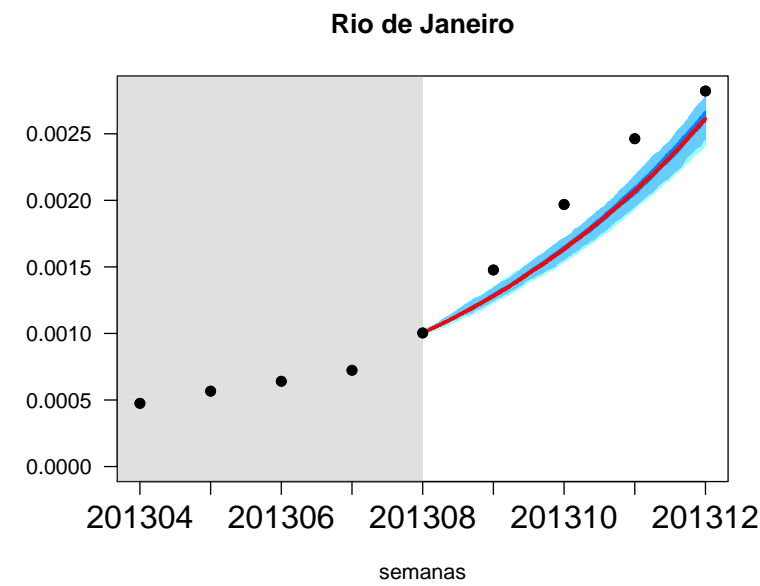

(d) $\tau=0.001$

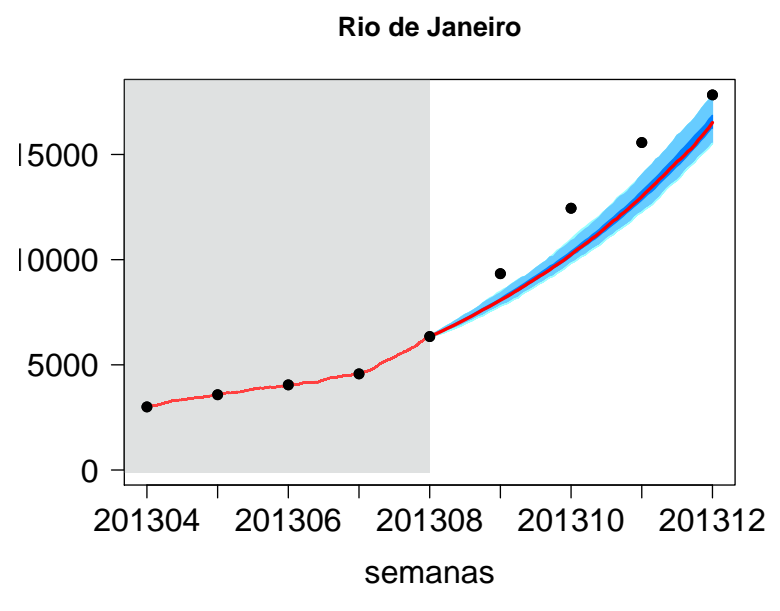

(f) Gillespie

Figura 5.1: Projeções geradas pela aproximação por processo difusivo para a cidade do Rio de Janeiro com diferentes tamanhos de passo temporal $(\tau)$. Os parâmetros adotados foram: $\hat{\beta}_{g_{1}}=1.132$ e $\hat{\gamma}_{g_{1}}=0.8847$ 


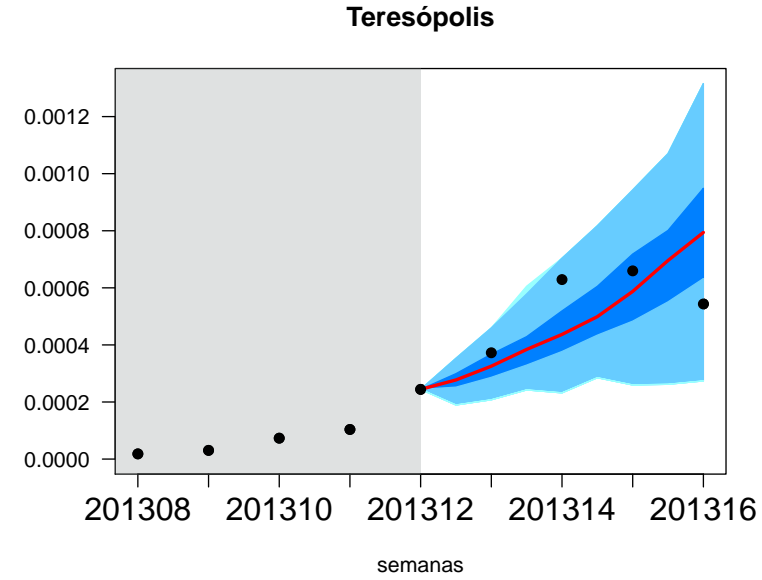

(a) $\tau=0.5$

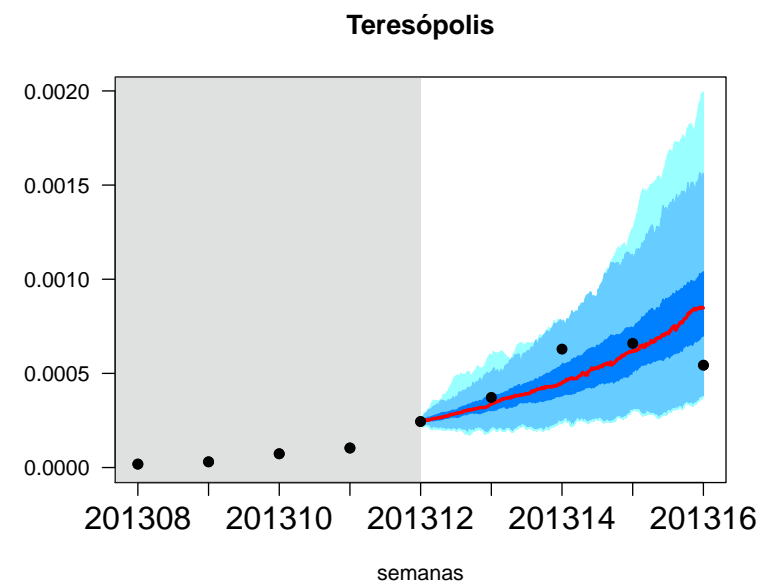

(c) $\tau=0.01$

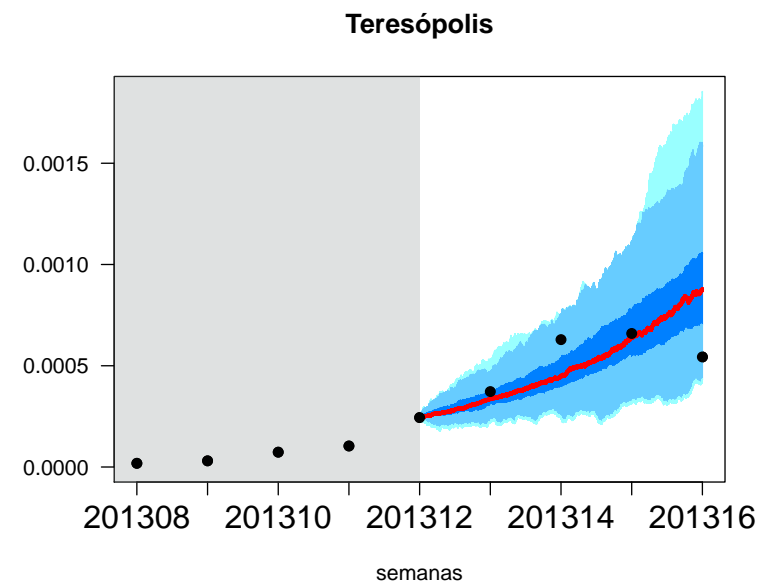

(e) $\tau=0.0001$

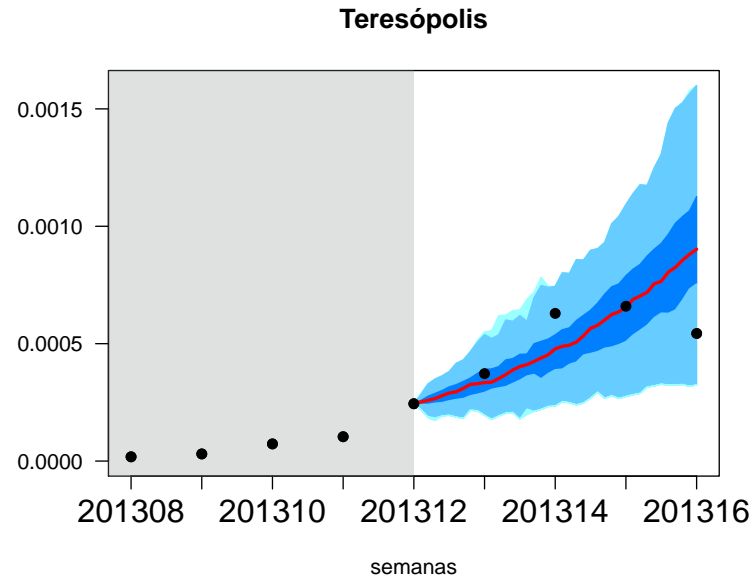

(b) $\tau=0.1$

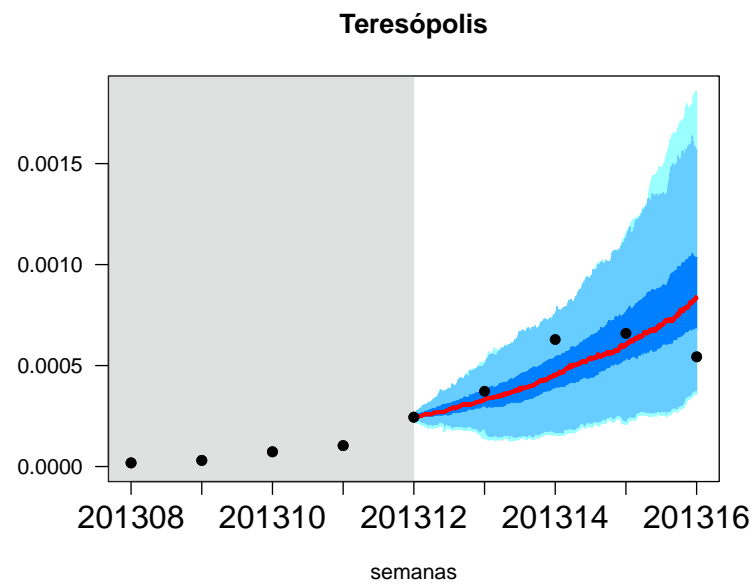

(d) $\tau=0.001$

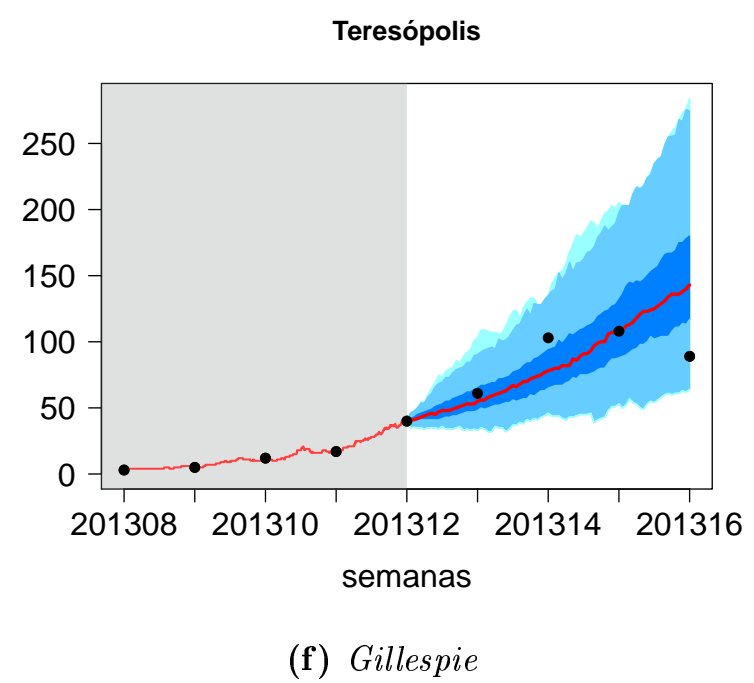

Figura 5.2: Projeções geradas pela aproximação por processo difusivo para a cidade de Teresópolis com diferentes tamanhos de passo temporal $(\tau)$. Os parâmetros adotados foram: $\hat{\beta}_{g_{2}}=1.177$ e $\hat{\gamma}_{g_{2}}=0.8567$ 


\section{Capítulo 6}

\section{Discussão}

Vamos agora relembrar a nossa jornada ao longo do nosso trabalho.

Nesse trabalho foi apresentado um modelo estocástico que descreve a evolução temporal de uma epidemia em sua fase inicial, inspirado nas interações entre as partículas de diversas substâncias em uma reação química. Nesse contexto, apresentamos o Stochastic Simulation Algorithm (SSA) ou algoritmo de Gillespie, modelo apresentado por Daniel Gillespie em seu artigo em 1977 [Gil77]. Nesse modelo, o instante de ocorrência e o tipo da próxima da próxima reação são regidas por leis estocásticas, que por sua vez são governadas por elementos conhecidos como funções de propensidade. A independência entre as probabilidades de escolha do tipo de reação e da determinação do instante da mesma, torna a simulação de um sistema através do algoritmo de Gillespie bastante simples.

Evidenciamos a dificuldade da inferência de parâmetros através de dados reais, exibindo primeiro o cenário idealizado em que se conhece o instante de todas as infecções e recuperações do sistema. Logo após, discutimos técnicas para inferir parâmetros através de observações discretas do estado do sistema. Tais estimadores foram deduzidos utilizando aproximações lineares das chamadas funções de propensidade e das características de processos de Poisson homogêneo e não-homogêneo.

Os estimadores foram inferidos através de dados de infecções por dengue no estado do Rio de Janeiro no período entre os anos 2012 e 2013, obtidos através do sistema SINAN [SIN16] do Ministério da Saúde do Brasil.

Através dos parâmetros estimados exibimos uma aplicação do algoritmo de Gillespie, onde foram projetados cenários para as semanas seguintes à semana de ajuste. O modelo é destinado a descrever a evolução de uma epidemia em sua fase inicial, uma vez que o avanço acelerado do número de notificações de uma doença gera preocupação e costumam ser acompanhadas de intervenções do governo ou autoridades médicas e sanitárias, alterando a dinâmica da doença.

Infelizmente, o modelo de Gillespie se mostra custoso computacionalmente, principalmente em casos em que há um número elevado de infecções (em grandes cidades, por exemplo). Para contornar esse problema exibimos a dedução de modelos como o Tau-leaping e o modelo difusivo (Langevin) que aproximam o algoritmo proposto por Gillespie.

Verificamos através de gráficos apresentados que o modelo difusivo apresentado no capítulo 5 capturam a tendência das projeções dos cenários futuros apresentados pelo modelo de Gillespie, sendo assim uma boa alternativa.

A principal vantagem da abordagem estocástica adotada nesse trabalho consiste no fato de que ao contrário dos modelos determinísticos que exibe uma curva única de projeção do estado do sistema ao longo do tempo, temos projeções dadas por faixas de probabilidade, que indicam o quão provável o sistema se encontrará em um determinado ponto.

\subsection{Um olhar para o horizonte}

Durante a pesquisa, muitas simplificações foram adotadas devido a ausência de dados disponíveis. Assim que dados que detalham o sorotipo de cada infecção sejam disponibilizados, uma 
possível extensão desse trabalho seria a inclusão de diferentes sorotipos da dengue na dinâmica do sistema. Nesse caso, uma infecção de cada sorotipo desencadearia uma reação de um tipo diferente o que aumentaria a complexidade do sistema.

Por outro lado, a disseminação de técnicas para a estimação do número de mosquitos $\left[\mathrm{BGN}^{+} 00\right.$, $\mathrm{dFBdCC}^{+} 01$ ] podem promover a elaboração de modelos mais detalhados, incluindo a dinâmica dos vetores na doença. Nesse caso teríamos a infecção de um mosquito descrita por uma reação que ocorre através do encontro entre um indivíduo infectado e um mosquito suscetível. Já a infecção de um humano seria descrita pelo encontro entre um indivíduo suscetível e um mosquito infectado.

Outra dificuldade enfrentada é a inexistência de critérios explícitos para a determinação do início da epidemia: o instante em que o regime letárgico (com pequeno número de notificações) é quebrado e o período de expansão da doença se inicia. Em nosso trabalho, a escolha do instante inicial se deu de forma manual, através de observação da evolução do número de notificações. A introdução de mecanismos automatizados de decisão e escolha para a semana inicial contribuiria de forma significativa para os modelos aqui apresentados. Identificando a semana inicial poderíamos inclusive, em uma visão mais ambiciosa, propor um modelo para tentar realmente prever o comportamento futuro do sistema, corrigindo as projeções do modelo semanalmente conforme novas observações puderem ser obtidas.

Em nossa pesquisa, propomos aproximações do algoritmo de Gillespie e atingimos o modelo difusivo baseado em equações diferenciais estocásticas, que foi abordado de forma superficial. Uma possível abordagem seria buscar estimadores para o modelo difusivo a partir dos dados de forma direta. Entretanto, isso requereria um aprofundamento maior na teoria de equações diferenciais estocásticas e do cálculo estocástico, que compreende uma rica e vasta área de pesquisa. Um aprofundamento na teoria pode ser encontrado no trabalho de Evans [Eva12] e Øksendal [Øks03]. Já para a parte numérica e simulações são recomendados os trabalhos de Higham [Hig01] e de Sauer [Sau12], este último com um enfoque maior em aplicações em finanças. A estimação de parâmetros é longamente discutida no trabalho de Fuchs [Fuc13] onde são discutidas diversas técnicas envolvendo aproximações da função de verossimilhança ou métodos alternativos, como por exemplo, a aplicação da metodologia MCMC com a utilização de pontes Brownianas (Brownian bridge). 


\section{Apêndice A}

\section{Processos Estocásticos}

Vamos aqui discutir brevemente alguns conceitos básicos da teoria de processos estocásticos que serão necessários em nosso trabalho.

As definições e a notação empregada seguem adjacentes aos encontrados nos livros de Hoel, Port e Stone de 1972 [HPS72], de Florescu de 2014 [Flo14] e de Sheldon Ross de 1996 [Ros96] e do trabalho de Huisinga e Meerbach de 2001 [HM05].

\section{A.1 Processo de Poisson}

Consideremos um espaço de probabilidade $(\Omega, \mathcal{A}, P)$, em que $\Omega$ denota o espaço amostral, $\mathcal{A}$ uma $\sigma$-álgebra e $P$ uma medida de probabilidade em $\Omega$. Podemos definir um processo estocástico como qualquer coleção de variáveis aleatórias definida nesse espaço de probabilidade. Mais especificamente, é a coleção de variáveis aleatórias $\{X(t): t \in \mathcal{I}\}$, onde $\mathcal{I}$ é um conjunto de índices.

O espaço domínio de todas as variáveis aleatórias $X(t)$ é denominado espaço de estados e será representado por $\mathcal{S}$. Tendo em vista nossas aplicações, vamos definir $\mathcal{I}=[0,+\infty]$. Um processo estocástico com essas características é denominado processo estocástico em tempo contínuo.

Para dados alguns $\omega \in \Omega$, o conjunto $\{X(t, \omega): t \geq 0\}$ com valores em $\mathcal{S}$ é denominada uma trajetória ou realização do processo estocástico $X$ associado a $\omega$.

Um processo estocástico em tempo contínuo $\{X(t): t \geq 0\}$ em um espaço de estados finito $\mathcal{S}$ é chamado de processo Markoviano de tempo contínuo, ou simplesmente processo Markoviano, ou mesmo cadeia de Markov se, para todo $s_{1}<\ldots<s_{k}<s<t$ e $x_{1}, \ldots, x_{k}, x, y \in \mathcal{S}$, a igualdade:

$$
P\left[X(t)=y \mid X\left(s_{1}\right)=x_{1}, \ldots, X\left(s_{k}\right)=x_{k}, X(s)=x\right]=P[X(t)=y \mid X(s)=x]
$$

é satisfeita.

A expressão A.1, define o que é conhecido como propriedade Markoviana. Essa característica, comumente chamada de perda de memória, indica que o desenvolvimento futuro do processo é incondicional ao passado.

Um processo Markoviano é dito homogêneo quando o lado direito da expressão A.1 depende apenas do tamanho do incremento $t-s$ (independe do estado do processo no instante $t$ ). Caso contrário, o processo é dito não homogêneo.

Dizemos que um processo estocástico $\{N(t): t \geq 0\}$ é um processo de contagem se $N(t)$ representa o número de eventos ocorridos até o instante $t$. Dessa forma, $N(t)$ deve satisfazer:

(a) $N(t)$ é um inteiro não negativo;

(b) Se $s<t$, então $N(s) \leq N(t)$;

(c) Para $s<t, N(t)-N(s)$ é igual ao número de eventos ocorridos no intervalo $] s, t]$. 
Um processo de contagem $N(t)$ possui incrementos independentes se para quaisquer $0<t_{1}<$ $\ldots<t_{n-1}<t_{n}$ temos que $N(0), N\left(t_{1}\right)-N(0), \ldots, N\left(t_{n}\right)-N\left(t_{n-1}\right)$ são variáveis aleatórias independentes.

Além disso, dizemos que um processo de contagem $N(t)$ possui incrementos estacionários se o número de eventos ocorridos em um intervalo $[t, t+s]$ depende apenas do comprimento do intervalo de tempo $s$, ou seja: $P[N(t+s)-N(s)=k]=P[N(t)=k]$.

Definição A.1.1. Processo de Poisson (homogêneo)

$N(t)$ é um processo de Poisson se é um processo de contagem e satisfaz:

(a) $N(0)=0$

(b) O processo possui incrementos independentes;

(c) O número de eventos em um intervalo de comprimento $t$ tem distribuição Poisson com média $\lambda t$ :

$$
P[N(t+s)-N(s)=n]=P[N(t)=n]=\frac{(\lambda t)^{n}}{n !} e^{-\lambda t} .
$$

onde o parâmetro $\lambda$ no item (c) é chamado de taxa de chegada (ou simplesmente taxa) do processo de Poisson.

Notemos que a nomenclatura dada ao processo provém da característica apresentada no item (c) da definição A.1.1.

Conforme demonstrado no livro de Ross [Ros96], o processo de Poisson pode alternativamente ser definido da seguinte forma:

Definição A.1.2. Processo de Poisson (homogêneo) - versão alternativa

Um processo $N(t)$ é dito de Poisson (homogêneo) com taxa de chegada $\lambda$ se é um processo de contagem e satisfaz as seguintes condiçôes adicionais:

(a) $N(0)=0$;

(b) $N(t)$ possui incrementos estacionários e independentes;

(c) $P[N(h)=1]=\lambda h+o(h)$;

(d) $P[N(h) \geq 2]=o(h)$,

onde uma função $f$ é dita o(h) se:

$$
\lim _{h \rightarrow 0} \frac{f(h)}{h}=0 .
$$

Vamos agora, construir a sequência $\left\{X_{i}, i=1,2, \ldots\right\}$ dos tempos de espera de um processo de Poisson:

$$
\begin{aligned}
X_{1} & =\text { tempo para o primeiro evento } \\
X_{2} & =\text { tempo entre o primeiro e o segundo eventos } \\
& \vdots \\
X_{n} & =\text { tempo entre o }(n-1) \text {-ésimo e o } n \text {-ésimo eventos }
\end{aligned}
$$


Através dela definimos $S_{n}$, o tempo até o $n$-ésimo evento, chamado também de $n$-ésimo tempo de chegada da seguinte forma:

$$
S_{n}=\sum_{i=0}^{n} X_{i}=\inf \{t \mid N(t) \geq n\}=\inf \{t \mid N(t)=n\}
$$

A figura A.1 exemplifica um processo de contagem.

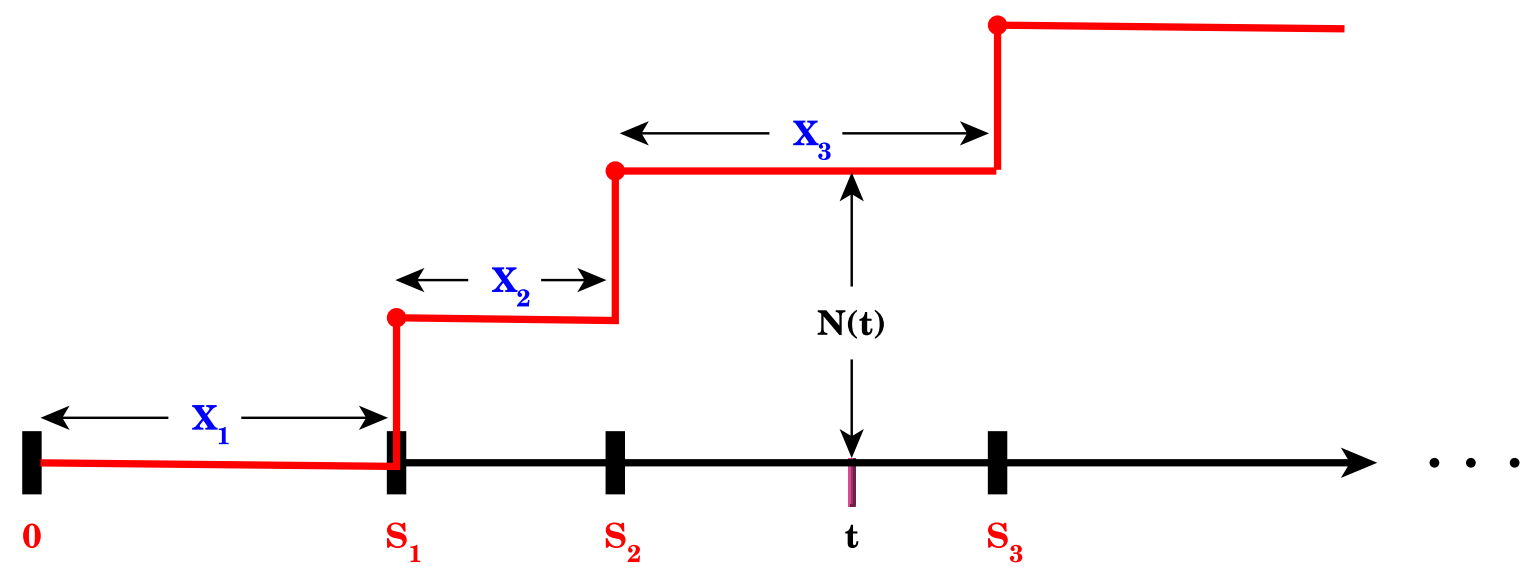

Figura A.1: Processo de contagem $N(t):\left\{S_{1}, S_{2}, \ldots\right\}$ representam os instantes $e\left\{X_{1}, X_{2}, \ldots\right\}$ representam os tempos intervalares entre as reações

Proposição A.1.3. Se $N(t)$ é um processo de Poisson com taxa $\lambda$, então os tempos intervalares entre as reações $X_{1}, X_{2}, \ldots$ são independentes e tem distribuição exponencial com parâmetro $\lambda$ $\left(X_{i} \sim \operatorname{Exp}(\lambda), i=1,2, \ldots\right)$.

Demonstração. Notemos que $P\left(X_{1}>t\right)$ é igual à probabilidade de não ocorrer nenhum evento do processo de Poisson no intervalo $[0, t]$. Daí:

$$
P\left(X_{1}>t\right)=P(N(t)=0)=e^{-\lambda t}
$$

Logo, $X_{1}$ possui função de distribuição dada por $F(t)=1-e^{-\lambda t}$. Portanto, $X_{1} \sim \operatorname{Exp}(\lambda)$.

Além disso,

$$
\begin{aligned}
P\left(X_{2}>t \mid X_{1}=s\right) & \left.=P(\text { nenhum evento ocorre em }] s, s+t] \mid X_{1}=s\right) \\
& =P(\text { nenhum evento ocorre em }] s, s+t]) \\
& =e^{-\lambda t}
\end{aligned}
$$

onde a igualdades A.3 e A.4 decorrem do fato dos incrementos serem independentes e estacionários em um processo de Poisson.

Logo, $X_{2} \sim \operatorname{Exp}(\lambda)$.

Aplicando o mesmo argumento para os demais pontos obtemos o resultado desejado.

Notemos primeiramente que há uma equivalência entre os eventos: $\{N(t) \geq n\}$ e $\left\{S_{n} \leq t\right\}$. Então:

$$
P\left(S_{n} \leq t\right)=P(N(t) \geq n)=\sum_{j=n}^{\infty} e^{-\lambda t} \frac{(\lambda t)^{j}}{j !}
$$


Diferenciando em $t$, obtemos a função densidade de $S_{n}$, temos:

$$
f_{S_{n}}(t)=\lambda e^{-\lambda t} \frac{(\lambda t)^{n-1}}{(n-1) !}
$$

Concluímos então que $S_{n}$ tem distribuição $\operatorname{Gamma}(n, \lambda)$.

Proposição A.1.4. Sejam $N_{1}(t)$ e $N_{2}(t)$ processos de Poisson independentes com taxas $\lambda_{1}$ e $\lambda_{2}$, respectivamente. Então $N(t)=N_{1}(t)+N_{2}(t)$ é também um processo de Poisson com taxa $\lambda_{1}+\lambda_{2}$.

Demonstração.

$$
P(N=n)=P\left(N_{1}+N_{2}=n\right)=\sum_{x=0}^{n} f_{N_{1}}(x) f_{N_{2}}(n-x)
$$

Então:

$$
\begin{aligned}
f_{N}(n) & =\sum_{x=0}^{n} \frac{\left(\lambda_{1} t\right)^{x}}{x !} e^{-\lambda_{1} t} \frac{\left(\lambda_{2} t\right)^{n-x}}{(n-x) !} e^{-\lambda_{2} t}=\left[\sum_{x=0}^{n} \frac{n !}{x !(n-x !)} \lambda_{1}^{x} \lambda_{2}^{n-x} t^{n}\right] \frac{e^{-\left(\lambda_{1}+\lambda_{2}\right) t}}{n !} \\
& =\left[\sum_{x=0}^{n}\left(\begin{array}{l}
x \\
n
\end{array}\right) \lambda_{1}^{x} \lambda_{2}^{n-x} t^{n}\right] \frac{e^{-\left(\lambda_{1}+\lambda_{2}\right) t}}{n !}=\left[\left(\lambda_{1}+\lambda_{2}\right) t\right]^{n} \frac{e^{-\left(\lambda_{1}+\lambda_{2}\right) t}}{n !}
\end{aligned}
$$

Portanto $N$ é um processo de Poisson de taxa $\left(\lambda_{1}+\lambda_{2}\right)$.

Vamos agora introduzir uma generalização do processo de Poisson em que a taxa de chegada do processo é uma função do tempo $t$.

Definição A.1.5. Processo de Poisson não homogêneo

Um processo de contagem $\{N(t), t \geq 0\}$ é dito processo de Poisson não homogêneo com função de intensidade $\lambda(t), t \geq 0$ se:

(a) $N(0)=0$;

(b) $\{N(t), t \geq 0\}$ possui incrementos independentes;

(c) $P[N(t+h)-N(t)=1]=\lambda(t) h+o(h)$;

(d) $P[N(t+h)-N(t) \geq 2]=o(h)$. 


\section{Apêndice B}

\section{Equações Diferenciais Estocásticas e Movimento Browniano}

Apresentaremos nessa seção uma pequena introdução à teoria de equações diferenciais estocásticas. O objetivo é apenas familiarizar suficientemente o leitor a assuntos abordados em nosso trabalho de forma a facilitar o entendimento. A notação empregada é próxima da adotada nos trabalhos de Evans [Eva12], Higham [Hig01] e Sauer [Sau12].

Um dos pontos centrais na teoria de equações diferenciais estocásticas é o conceito de Movimento Browniano:

Um movimento Browniano padrão (standard Brownian motion) escalar, ou processo de Wiener padrão (standard Wiener process), sobre um intervalo $[0, T]$ é uma variável aleatória $W(t)$ que depende continuamente de $t \in[0, T]$ e satisfaz três condições:

(1) $P(W(0)=0)=1$;

(2) Para $0 \leq s<t \leq T$, a variável aleatória definida pelo incremento $W(t)-W(s)$ tem distribuição Normal com média zero e desvio padrão $t-s$. Equivalentemente: $W(s) \sim \sqrt{t-s} Z$, onde $Z \sim \operatorname{Normal}(0,1)$;

(3) Para $0 \leq s<t<u<v \leq T$, os incrementos $W(t)-W(s)$ e $W(v)-W(u)$ são independentes.

O nome processo de Wiener é dado em homenagem ao matemático Norbert Wiener que, construiu e formalizou o comportamento aleatório caracterizado pelo botânico Robert Brown em 1827, comumente chamado de movimento Browniano [Sau12]. Ele pode ser rigorosamente definido através do limite de passeios aleatórios quando os tamanhos dos passos espacial e temporal vão ambos a zero. Movimentos Brownianos são cruciais na modelagem de processos estocásticos uma vez que representam a integral de um ruído idealizado, conhecido como ruído branco (white noise). Frequentemente os processos de Wiener são empregados para representar aleatoriedade ou influências externas em um sistema determinístico [Sau12].

Para propostas computacionais é interessante considerar um movimento Browniano discretizado, em que $W(t)$ é especificado em um conjunto discreto valores [Hig01]. Tomando um inteiro $N$ e definindo $\delta t=T / N$, usaremos a notação $W_{j}$ para denotar $W\left(t_{j}\right)$, onde $t_{j}=j \delta t$. A condição 1 nos fornece $W_{0}=0$. Já das condições 2 e 3 temos:

$$
W_{j}=W_{j-1}+d W_{j}, \quad j=1,2, \ldots, N
$$

onde cada $d W_{j}$ é uma variável aleatória independente na forma: $\sqrt{\delta t} Z \operatorname{com} Z \sim \operatorname{Normal}(0,1)$. 


\section{B.1 Integrais Estocásticas}

Uma equação diferencial estocástica escalar e autônoma pode ser escrita na forma (forma integral):

$$
X(t)=X_{0}+\int_{0}^{T} a(X(s)) d s+\int_{0}^{T} b(X(s)) d W(s), \quad 0 \leq t \leq T
$$

onde $a$ e $b$ são funções escalares e a condição inicial $X_{0}$ é uma variável aleatória. A última integral de B.1 é tomada com respeito ao movimento Browniano, através da integral de Ito, que será definida posteriormente. A solução $X(t)$ é uma variável aleatória para cada $t \in[0, T]$.

A expressão B.1 é comumente reescrita em sua forma diferencial:

$$
\left\{\begin{array}{l}
X(0)=X_{0} \\
d X(t)=a(X(t)) d t+b(X(t)) d W(t), \quad 0 \leq t \leq T
\end{array}\right.
$$

Notemos que não nos é permitido escrever $d W(t) / d t$ uma vez que o movimento Browniano não é diferenciável em nenhum ponto [Hig01]. Observemos ainda que tomando $b \equiv 0$ e $X_{0}$ constante, obtemos o problema determinístico definido por: $X(0)=X_{0}, d X(t) / d t=a(X(t))$.

Antes de definir a integral de Ito, notemos primeiramente que, dada uma função contínua qualquer $f$ definida no intervalo $[0, T]$, a integral de Riemann é definida como o limite:

$$
\int_{0}^{T} f(t) d t=\lim _{\Delta t \rightarrow 0} \sum_{i=1}^{N} f\left(t_{i}^{\prime}\right) \Delta t_{i}
$$

onde $t_{i}^{\prime} \in\left[t_{i-1}, t_{i}\right]$ e $\Delta t_{i}=t_{i}-t_{i-1}$.

De modo bastante similar podemos definir a integral de Ito como o limite:

$$
\int_{0}^{T} f(t) d W(t)=\lim _{\Delta t \rightarrow 0} \sum_{i=1}^{N} f\left(t_{i-1}\right) \Delta W_{i}
$$

Notemos que equanto na integral de Riemann o argumento $t_{i}^{\prime}$ de $f$ é um ponto qualquer tomado no intervalo $\left[t_{i-1}, t_{i}\right]$, no caso da integral de Ito, o argumento de $f$ é o ponto extremo esquerdo do intervalo supracitado [Sau12].

\section{B.2 Método de Euler-Maruyama}

Um dos métodos mais simples para aproximação de equações diferenciais ordinárias é o método de Euler. Nessa seção, apresentaremos o método de Euler-Maruyama, uma adaptação do método de Euler para aproximação de equações diferenciais estocásticas.

Dado o problema de valor inicial definido em B.2, o método de Euler-Maruyama calcula a solução aproximada do sistema da seguinte forma:

$$
\left\{\begin{array}{l}
w_{0}=X_{0} \\
w_{i+1}=w_{i}+a\left(w_{i}\right) \Delta t_{i+1}+b\left(w_{i}\right) \Delta W_{i+1} \quad i=0,1, \ldots, N-1
\end{array}\right.
$$

onde $w_{i}$ representa a aproximação obtida no ponto $X\left(t_{i}\right)$, e 


$$
\begin{aligned}
\Delta t_{i+1} & =t_{i+1}-t_{i} \\
\Delta W_{i+1} & =W_{i+1}-W_{i}
\end{aligned}
$$

Lembrando das propriedades discutidas anteriormente, temos:

$$
\Delta W_{i}=z_{i} \sqrt{\Delta t_{i}}, \quad \text { onde } z_{i} \sim \operatorname{Normal}(0,1)
$$

Temos então, que o método de Euler-Maruyama nos fornece uma maneira bastante simples de obter a aproximação de um problema de valor inicial para uma equação diferencial estocástica.

Há diversos outros métodos de obter essas aproximações, com diferentes ordens de convergência. Vamos nos ater ao método de Euler-Maruyama, que será suficiente aos nossos propósitos. Ao leitor interessado em conhecer outros métodos recomendamos consultar ao trabalhos de Higham [Hig01] e de Sauer [Sau12]. 
APÊNDICE B 


\section{Apêndice C}

\section{A fórmula de Taylor e a representação de derivadas}

Em métodos numéricos, é comum o emprego da expansão em séries de Taylor para a obtenção de uma fórmula para a derivada. Essas fórmulas são especialmente úteis no capítulo 5.

Primeiramente, consideremos as expansões em série de Taylor para funções de uma variável:

$$
\begin{aligned}
& f(x+h)=f(x)+h f^{\prime}(x)+\frac{h^{2}}{2 !} f^{\prime \prime}(x)+\frac{h^{3}}{3 !} f^{\prime \prime \prime}(x)+\ldots, \quad h>0 \\
& f(x-h)=f(x)-h f^{\prime}(x)+\frac{h^{2}}{2 !} f^{\prime \prime}(x)-\frac{h^{3}}{3 !} f^{\prime \prime \prime}(x)+\ldots, \quad h<0
\end{aligned}
$$

Isolando $f^{\prime}(x)$ da expressões em C.1 e C.2 obtemos uma aproximações para a derivada:

$$
\begin{aligned}
& f^{\prime}(x)=\frac{f(x+h)-f(x)}{h}-\sum_{i=2}^{\infty} \frac{h^{i-1}}{i !} f^{(i)}(x) \\
& f^{\prime}(x)=\frac{f(x)-f(x-h)}{h}+\sum_{i=2}^{\infty} \frac{(-1)^{i} h^{i-1}}{i !} f^{(i)}(x)
\end{aligned}
$$

Tomando $h$ suficientemente pequeno, podemos escrever:

$$
f^{\prime}(x) \approx \frac{f(x+h)-f(x)}{h} \quad \text { e } \quad f^{\prime}(x) \approx \frac{f(x)-f(x-h)}{h}
$$

Então, para funções de duas variáveis $f(x, y)$ e tomando $h, k \rightarrow 0$, obtemos:

$$
\begin{array}{ccc}
\frac{\partial}{\partial x} f(x, y) \approx \frac{f(x+h, y)-f(x, y)}{h} & \text { e } \frac{\partial}{\partial x} f(x, y) \approx \frac{f(x, y)-f(x-h, y)}{h} \\
\frac{\partial}{\partial y} f(x, y) \approx \frac{f(x, y+k)-f(x, y)}{k} & \text { e } \frac{\partial}{\partial y} f(x, y) \approx \frac{f(x, y)-f(x, y-k)}{k}
\end{array}
$$

Já em ordem superior, podemos considerar: 


$$
\begin{aligned}
\frac{\partial^{2}}{\partial y \partial x} f(x, y) & =\frac{\partial}{\partial y}\left(\frac{\partial}{\partial x} f(x, y)\right) \\
& \approx \frac{\partial}{\partial y}\left(\frac{f(x+h, y)-f(x, y)}{h}\right) \\
& \approx \frac{f(x+h, y+k)-f(x, y+k)}{h k}-\frac{f(x+h, y)-f(x, y)}{h k} \\
& =\frac{f(x+h, y+k)-f(x, y+k)-f(x+h, y)+f(x, y)}{h k}
\end{aligned}
$$

De modo análogo:

$$
\begin{aligned}
\frac{\partial^{2}}{\partial x^{2}} f(x, y) & \approx \frac{\partial}{\partial x}\left(\frac{f(x+h, y)-f(x, y)}{h}\right) \\
& \approx \frac{f(x+h, y)-f(x, y)-f(x, y)+f(x-h, y)}{h^{2}} \\
& =\frac{f(x+h, y)-2 f(x, y)+f(x-h, y)}{h^{2}}
\end{aligned}
$$




\section{Referências Bibliográficas}

[Ald97] John Aldrich. Ra fisher and the making of maximum likelihood 1912-1922. Statistical science, páginas 162-176, 1997. 14

[BFJ14] Andrew S Boozary, Paul E Farmer e Ashish K Jha. The Ebola outbreak, fragile health systems, and quality as a cure. Jama, 312(18):1859-1860, 2014. 1

$\left[\mathrm{BGB}^{+} 13\right]$ Samir Bhatt, Peter W Gething, Oliver J Brady, Jane P Messina, Andrew W Farlow, Catherine L Moyes, John M Drake, John S Brownstein, Anne G Hoen, Osman Sankoh et al. The global distribution and burden of dengue. Nature, 496(7446):504-507, 2013. 5

[BGN $\left.{ }^{+} 00\right]$ Ima Aparecida Braga, Almério de Castro Gomes, Michel Nelson, Rita de Cassia G Mello, Denise Pimentel Bergamaschi e José Maria Pacheco de Souza. Comparação entre pesquisa larvária e armadilha de oviposição, para dectecção de Aedes aegypti. Rev Soc Bras Med Trop, páginas 347-353, 2000. 4, 58

[Bul16] Lorena Carvalho Bulhosa. Dinâmica de uma epidemia com migração de indivíduos, jul 2016. 49

[BV07] Ima Aparecida Braga e Denise Valle. Aedes aegypti: histórico do controle no brasil. Epidemiologia e serviços de saúde, 16(2):113-118, 2007. 3

[BWK08] Richard J Boys, Darren J Wilkinson e Thomas BL Kirkwood. Bayesian inference for a discretely observed stochastic kinetic model. Statistics and Computing, 18(2):125-135, 2008. 20

[Dar07] Christiane Dargatz. A diffusion approximation for an epidemic model. Relatório técnico, Discussion paper//Sonderforschungsbereich 386 der Ludwig-Maximilians-Universität München, 2007. 47, 48, 50

[dFBdCC $\left.{ }^{+} 01\right]$ Eudina AM de Freitas Barata, Antônio Ismael P da Costa, Francisco Chiaravalloti, Carmen M Glasser, José Maria S Barata e Delsio Natal. População de aedes aegypti (1.) em área endêmica de dengue, Sudeste do Brasil. Rev Saúde Pública, 35(3):237-42, 2001. 4,58

[Edw74] Anthony WF Edwards. The history of likelihood. International Statistical Review/Revue Internationale de Statistique, páginas 9-15, 1974. 14

[Eva12] Lawrence C Evans. An introduction to stochastic differential equations, volume 82. American Mathematical Soc., 2012. 58, 63

[Fis22] Ronald A Fisher. On the mathematical foundations of theoretical statistics. Phil. Trans. R. Soc. Lond. A, 222(594-604):309-368, 1922. 14

[Fis97] Ronald A Fisher. On an absolute criterion for fitting frequency curves. Statistical Science, 12(1):39-41, 1997. 14 
[Flo14] Ionut Florescu. Probability and Stochastic Processes. John Wiley \& Sons, 2014. 9, 59

[Fuc13] Christiane Fuchs. Inference for Diffusion Processes: With Applications in Life Sciences. Springer Science \& Business Media, 2013. 47, 48, 50, 51, 58

[Gil77] Daniel T Gillespie. Exact stochastic simulation of coupled chemical reactions. The journal of physical chemistry, 81(25):2340-2361, 1977. 57

[Gil08] Daniel Gillespie. Simulation methods in systems biology. Formal Methods for Computational Systems Biology, páginas 125-167, 2008. xv, 6, 7, 9, 51, 53

$\left[\mathrm{GSP}^{+}{ }^{10}\right]$ Gissel García, Beatriz Sierra, Ana B Pérez, Eglys Aguirre, Ileana Rosado, Narjara Gonzalez, Alienys Izquierdo, Maritza Pupo, Didye Ruiz Danay Díaz, Lizet Sánchez et al. Asymptomatic dengue infection in a Cuban population confirms the protective role of the rr variant of the fc $\gamma$ riia polymorphism. The American journal of tropical medicine and hygiene, 82(6):1153-1156, 2010. 5

[Gup13] Ankur Gupta. Parameter estimation in deterministic and stochastic models of biological systems. Tese de Doutorado, The University of Wisconsin-Madison, 2013. 6, 7, 16

[Het00] Herbert W Hethcote. The mathematics of infectious diseases. SIAM review, 42(4):599653, 2000. 11

[Hig01] Desmond J Higham. An algorithmic introduction to numerical simulation of stochastic differential equations. SIAM review, 43(3):525-546, 2001. 58, 63, 64, 65

[Hig08] Desmond J Higham. Modeling and simulating chemical reactions. SIAM review, $50(2): 347-368,2008.6,7,8,9,51$

[HJ02] Michael Höhle e Erik Jørgensen. Estimating parameters for stochastic epidemics. [The Royal Veterinary and Agricultural University], Dina, 2002. 15

[HM05] Wilhelm Huisinga e Eike Meerbach. Markov processes for everybody. Introduction to the theory of continuous time Markov processes. Fachbereich Mathematik und Informatik. Freien Universität Berlin \& DFG Research Center Matheon, Berlin, 2005. 59

[HPS72] Paul G Hoel, Sidney C Port e Charles J Stone. Introduction to Stochastic Processes, 1972. 59

[IBBM ${ }^{+}$97] SERGIO IBÁÑEZ-BERNAL, Baltasar Briseno, John Paul Mutebi, Enid Argot, Guadalupe Rodriguez, CARMEN MARTINEZ-CAMPOS, Rafael Paz, PEDRO DE LA FUENTE-SAN ROMÁN, ROBERTO TAPIA-CONYER e Ana Flisser. First record in America of Aedes albopictus naturally infected with dengue virus during the 1995 outbreak at Reynosa, Mexico. Medical and veterinary entomology, 11(4):305-309, 1997. 3

[IBG10] IBGE. Ibge censo 2010. Acesso em, 23, 2010. Último acesso em 21/7/2017. xvii, 29, $30,34,39$

[IUP17] IUPAC. Compendium of Chemical Terminology, 2nd ed. (the "gold book"). https: //doi.org/10.1351/goldbook, ago 2017. 6, 7

[MB98] Lynda E Muir e H Kay Brian. Aedes aegypti survival and dispersal estimated by markrelease-recapture in northern Australia. 1998. 4

[MIC09] Dicionário da Língua Portuguesa MICHAELIS. Dicionário online uol. Editora Melhoramentos, 2009. 14 
[MT06] Pedro A Morettin e Clélia Toloi. Análise de séries temporais. Em Análise de séries temporais. 2006. 18

[Øks03] Bernt Øksendal. Stochastic differential equations. Em Stochastic differential equations, páginas 65-84. Springer, 2003. 58

[PTP98] Gerson Oliveira Penna, MG TEIXEIRA e SM PEREIRA. Doenças infecciosas e parasitárias: aspectos clínicos de vigilância epidemiológica e de controle-guia de bolso. Brasilia: Ministério da Saúde: Fundação Nacional de Saúde, 218, 1998. 4, 5

[RMVC06] Andressa F Ribeiro, Gisela RAM Marques, Júlio C Voltolini e Maria Lúcia F Condino. Associação entre incidência de dengue e variáveis climáticas. Revista de Saúde Pública, 40(4):671-676, 2006. 3

[Ros96] Sheldon M Ross. Stochastic processes, 1996. 59, 60

[RSO+17] Marco Aurélio Ruediger, Danielle Sanches, Wagner Oliveira, Andressa Contarato, Rachel Bastos, Janaina de Mendonça Fernandes e Bárbara Barbosa. Febre Amarela no Brasil: um estudo de caso. Relatório técnico, FGV DAPP, 2017. 1

[Sau12] Timothy Sauer. Numerical solution of stochastic differential equations in finance. Em Handbook of computational finance, páginas 529-550. Springer, 2012. 58, 63, 64, 65

[SF16] Lavinia Schuler-Faccini. Possible association between Zika virus infection and microcephaly_Brazil, 2015. MMWR. Morbidity and mortality weekly report, 65, 2016. 1

[SG09] Ying Sun e Marc G Genton. Functional boxplots for complex data visualization. 2009. 32

[SIN16] SINAN. Dados Epidemiológicos SINAN. "http://portalsinan.saude.gov.br/ dados-epidemiologicos-sinan", Novembro 2016. 1, 13, 17, 57

[SKHK09] Clive R Seed, Philip Kiely, Catherine A Hyland e Anthony J Keller. The risk of dengue transmission by blood during a 2004 outbreak in cairns, australia. Transfusion, 49(7):1482-1487, 2009. 5

[Tau01] Pedro Luiz Tauil. Urbanização e ecologia do dengue. 2001. 3, 4

[Tea14] WHO Ebola Response Team. Ebola virus disease in West Africa - the first 9 months of the epidemic and forward projections. New England Journal of Medicine, 371(16):14811495, 2014. 1

[TH86] Milan Trpis e Walter Hausermann. Dispersal and other population parameters of Aedes aegypti in an African village and their possible significance in epidemiology of vectorborne diseases. The American Journal of Tropical Medicine and Hygiene, 35(6):12631279, 1986. 4

[Ujv03] Stefan Cunha Ujvari. A história e suas epidemias: a convivência do homem com os microrganismos. Revista do Instituto de Medicina Tropical de São Paulo, 45(4):212212, 2003. 1

[Ver91] Ricardo Veronesi. Doenças infecciosas e parasitárias. Revista do Instituto de Medicina Tropical de São Paulo, 33(4):286-286, 1991. 4

[WHO17a] WHO. Dengue control. http://www.who.int/denguecontrol/human/en/, Julho 2017. 4

[WHO17b] WHO. ITH Webmap application (Countries or areas where dengue has been reported or at risk of dengue). http://apps.who.int/ithmap/, Junho 2017. Último acesso em 27/6/2017. xiii, 3 
[Wil11] Darren J Wilkinson. Stochastic modelling for systems biology. CRC press, 2011. 6, 14, $15,20,23$

[Yan03] HM Yang. Epidemiologia da transmissão da dengue. Trends in Applied and Computational Mathematics, 4(3):387-396, 2003. 4 


\section{Índice Remissivo}

Aedes, 3

aegypti, 3

albopictus, 3

algoritmo de Gillespie, 8

block updating method, 20

boxplot funcional, 32

cadeia de Markov, 6, 59

em tempo contínuo, 6

Chemical Master Equation, 7, 8, 47

cinética

química, 6

CME, veja Chemical Master Equation

DC, veja Dengue Clássica

dengue, 3

clássica, 4

hemorrágica, 4

densidade demográfica, 10

DH, veja Dengue Hemorrágica

diffusion term, 50

distribuição

exponencial, 9

gama, 16

posteriori, 16, 17

marginal, 17

priori, 16

conjugada, 16

doenças infecciosas, 1

drift term, 50

EMV, veja Estimador de Máxima Verossimilhança equação

de difusão progressiva de Kolmogorov, 50

de Fokker-Plank, 50

diferencial estocástica, 50, 63

mestra, 8

espaço

amostral, 59

de estados, 59

de probabilidade, 59

espécies químicas, 6

estimador de máxima verossimilhança, 14

FCD, veja Forma Clássica de Dengue

FHD, veja Forma Hemorrágica de Dengue

flavivírus, 3

flaviviridae, 3

forma

clássica da dengue, 4

diferencial da equação diferencial estocástica, 64

hemorrágica da dengue, 4

integral da equação diferencial estocástica, 64

fórmula de Bayes, 16

função

de propensidade, 6,8 separável, 7

densidade conjunta, 9

hazard, 6

hospedeiro, 4

infectado, 4,9

assintomático, 5

sintomático, 5, 13

infectante, 4

integral

de Ito, 64

de Riemann, 64

média móvel simples, 18

método

da tranformação inversa para geração de variável aleatória, 9

de Euler Maruyama, 64

de máxima verossimilhança, 14

Metropolis-Hastings update, 20

MMS, veja Média Móvel Simples

modelo

matemático, 3

moléculas, 6

movimento Browniano, 63

discretizado, 63 
outliers, 33

passeio aleatório, 63

perda de memória, 59

período

de ajuste, 5, 17

ponte Browniana, 58

população relativa, 10

problema de valor inicial, 64

processo

com incrementos estacionários, 60

com incrementos independentes, 60

de contagem, 59

de Poisson homogêneo, 60

de Poisson não homogêneo, 9, 62

de Wiener, 63

estocástico, 59

estocástico em tempo contínuo, 59

Markoviano

de tempo contínuo, 59

homogêneo, 59

não homogêneo, 59

propriedade Markoviana, 6, 59

reação, 6

bimolecular, 7

de dimerização, 7

de primeira ordem, 7

de segunda ordem, 7

elementar, 6

termolecular, 7

unimolecular, 6, 7

realização do processo estocástico, 59

recuperado, 9

ruído branco, 63

SCD, veja Síndrome de Choque da Dengue

séries de Taylor, 67

SINAN, veja Sistema de Informação de Agravos de Notificação

síndrome

de choque da dengue, 4

Sistema de Informação de Agravos de Notificação, 1

sorotipos, 3

SSA, veja Stochastic Simulation Algorithm

Stochastic Simulation Algorithm, 8

suavização, 18

suscetível, 4, 9

taxa de chegada, 60

tempo

de chegada, 61

de espera, 60 de recuperação, 11

termo

difusivo, 50

flutuante, 50

trajetória, 20, 59

viável, 20

variável

aleatória, 8

verossimilhança, 14

vetor, 4

de troca de estados, 6

estequiométrico, 6

Well-stirred system, 6, 9 